Portland State University

PDXScholar

Fall 1-3-2013

\title{
The Institutional Context that Supports Team-Based Care for Older Adults
}

Anna Foucek Tresidder

Portland State University

Follow this and additional works at: https://pdxscholar.library.pdx.edu/open_access_etds

Part of the Geriatrics Commons, Health and Medical Administration Commons, and the Health Services Administration Commons

Let us know how access to this document benefits you.

\section{Recommended Citation}

Tresidder, Anna Foucek, "The Institutional Context that Supports Team-Based Care for Older Adults" (2013). Dissertations and Theses. Paper 1517.

https://doi.org/10.15760/etd.1503

This Dissertation is brought to you for free and open access. It has been accepted for inclusion in Dissertations and Theses by an authorized administrator of PDXScholar. Please contact us if we can make this document more accessible: pdxscholar@pdx.edu. 
The Institutional Context that Supports Team-Based Care for Older Adults

by

Anna Foucek Tresidder

A dissertation submitted in partial fulfillment of the requirements for the degree of

Doctor of Philosophy

in

Health Systems and Policy

Dissertation Committee:

Sherril B. Gelmon, Chair

Paula Carder

Greg Schrock

Donald Truxillo

Portland State University

2013 
(C) 2013 Anna Foucek Tresidder 


\begin{abstract}
The aging population in the U.S. is dramatically increasing; it is predicted that not only will individuals live longer but also that they will live with multiple chronic diseases that could require high levels of medical and social resources. While the aging population increases, the number of health care providers choosing to specialize in caring for the elderly is decreasing just as dramatically. Teams are believed to be a possible response to more efficiently use the providers available, take advantage of alternative provider types, and integrate a range of health and social services to meet patient needs more effectively. Interdisciplinary teams are the best practice in the care of older adults, who require both medical and social services. However, maintaining functional collaborative teams has been an ever-present challenge to health and social care organizations. Research has found that institutional support is critical for teams to benefit patients and organizations alike.
\end{abstract}

This study examined the role of institutional context in supporting interdisciplinary teams (IDT) in the care of older adults through interviews of the management and staff of the Program for All-Inclusive Care of the Elderly (PACE) in six states. PACE organizations must commit to an interdisciplinary model of care consisting of 11 different disciplines from across the professional spectrum. The research question posed for this study was: What elements of institutional context support the use of interdisciplinary teams in the care of older adults? Due to the standardized team structure used, PACE was selected as the model to see how institutions at macro and micro levels 
support the work done by PACE teams and possibly highlight where support is still lacking. A case study approach drawing upon qualitative methods was used to examine policy-regulative, cultural-cognitive, normative, relational, and procedural elements of institutional context and the extent to which they support collaborative teamwork. Thirty-two interviews were conducted with administrators and team members from seven PACE programs across the country. For these PACE programs, five elements and 14 categories of support were identified by the interviewees.

Policy and regulatory elements constrain and systematize behavior. PACE IDT experience these constraints and systems through regulatory body practices, resource allocation, and quality measurement. Cultural-cognitive elements mediate between an IDT's external environment and the response of the IDT in order to make sense of what is happening. PACE IDTs create meaning through their interactions with their external environments through interdependence, demographic characteristics, and organizational structure. Normative findings constrain behavior and confer the rights and duties of IDT members, which arise from organizational mission and values, leadership, and professional boundaries. Relational elements emphasize relationships amongst IDT members and team interaction with the organization's environment. Social constructs within the team affect role definition and communication, which support IDT practice. Procedural support standardizes practices to maintain highly functional teams. In order to support IDT practice, PACE organizations highlighted recruitment and retention, time and space, and training and education as the primary ways to support IDTs. These categories illustrate the complexity of supporting teams and actualizing teamwork in 
practice. These findings suggest that PACE is succeeding in supporting the IDT model and provides lessons for other organizations that wish to do the same. 


\section{Dedication}

To Mike, Milana, Aila, and Freya

You are my inspiration and my reality check. You kept me focused and grounded throughout the entire process, and I could not have done it without your support.

\section{To Mom and Dad}

Everything I accomplish in life began with the strong foundation you built for me. Thank you.

I love you all more than words can express. 


\section{Acknowledgements}

I knew my dissertation journey was going to be unusual when I found out I was pregnant with twins and that I had been accepted into my Ph.D. program in the same week. I knew I wanted to pursue my Ph.D. but would need an army of support to realize the challenge. The outpouring of support that I received that made this all possible has humbled me.

First, I want to thank my chair Sherril Gelmon. We started this journey brainstorming research ideas in the St. Vincent maternity ward while I was on bed rest. We end this journey through difficult and challenging times, yet your commitment as my chair never wavered. I am so grateful for your encouragement, guidance and perseverance through it all.

My sincere appreciation goes to the other members of my committee, Paula Carder and Greg Schrock. Thank you for all your time, comments, and efforts in bringing a complex topic into focus. Thanks also to Donald Truxillo, my graduate office representative, who provided much more than required by his role. Thank you all for your support in seeing this project to a timely and successful conclusion.

Thanks also to members of faculty who provided guidance and support along the way: Neal Wallace, Craig Shinn, Diana White, and Rita Sumner. All of you played an instrumental role along the way. Your knowledge, encouraging words, and sage advice were invaluable. 
My Ph.D. cohort was full of amazing women. Phyusin, Mariah, Tiphaine, and Robyn you all inspired me to continue making progress and getting through the all the classes and comprehensive exams. It was an honor to be with you and I am grateful to have shared this journey with you.

I also could not have finished this without those who provided loving care to our wonderful children, so I could write, work, and attend class. Nana Sue, Grane Anne, and Poppy Don, your enthusiasm to share your time with our children is wonderful. We are so lucky and happy to have you in our lives. Thanks also to my emotional support Brooke, Tracy, Wendy, Sarah and Jinnet who all buoyed me when I was hindered by uncertainty.

I dedicated this work to my husband, children and parents. All played a crucial everyday role in inspiring me to persevere, even when I was plagued with doubts. It would not have been possible without the contribution from everyone and I will be eternally grateful. 


\section{Table of Contents}

Abstract $\quad$ i

Dedication $\quad$ iv

Acknowledgements $\quad$ V

List of Tables $\quad \mathrm{X}$

List of Figures $\quad$ xi

Chapter 1 Background 1

Introduction 1

Problem Statement $\quad 11$

Research Question $\quad 12$

Purpose of Study 12

Significance of Study 13

$\begin{array}{ll}\text { Conclusion } & 14\end{array}$

Chapter 2 Review of the Related Literature $\quad 15$

$\begin{array}{ll}\text { Introduction } & 15\end{array}$

Health and Social Policy Context 16

Organizational Institutionalism $\quad 24$

Professions and Institutions $\quad 35$

Interdisciplinary Teams in Health Care $\quad 37$

Conclusion $\quad 56$

Chapter 3 Methodology 58

Introduction $\quad 58$

Research Theory $\quad 58$

Research Question $\quad 59$

Research Rationale and Design $\quad 75$

Human Subjects Protection 76

$\begin{array}{ll}\text { Role of the Researcher } & 79\end{array}$ 
$\begin{array}{ll}\text { Data Collection } & 79\end{array}$

Data Analysis $\quad 84$

Trustworthiness of Research 86

$\begin{array}{ll}\text { Limitations } & 87\end{array}$

$\begin{array}{lr}\text { Conclusion } & 87\end{array}$

Chapter 4 Presentation and Analysis of Data $\quad 88$

Introduction $\quad 88$

Policy-Regulative Elements $\quad 89$

Cultural-Cognitive Elements 107

Normative Elements $\quad 123$

Relational Elements $\quad 137$

$\begin{array}{ll}\text { Procedural Elements } & 148\end{array}$

Summary of Findings $\quad 161$

Chapter 5 Discussion, Conclusions and Recommendations for Future Research 164

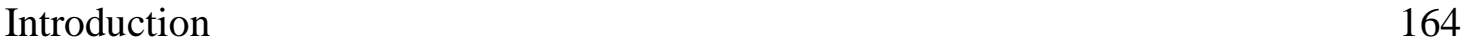

$\begin{array}{ll}\text { Policy-Regulative } & 167\end{array}$

$\begin{array}{ll}\text { Cultural-Cognitive } & 173\end{array}$

$\begin{array}{ll}\text { Normative Elements } & 179\end{array}$

Relational Elements $\quad 185$

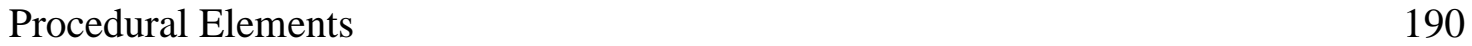

$\begin{array}{ll}\text { Overall Synthesis } & 194\end{array}$

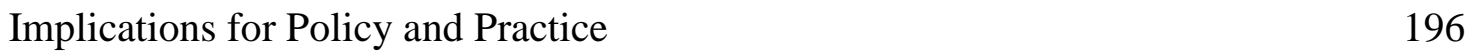

$\begin{array}{ll}\text { Assumptions and Limitations } & 199\end{array}$

Recommendations for Further Research $\quad 201$

$\begin{array}{ll}\text { Conclusions } & 204\end{array}$

Bibliography $\quad 207$

Appendix A: Human Subjects Research Review Committee (HSRRC) Application 223 Appendix B: Initial Email for Organizational Approval 230

Appendix C: Initial Email for Key Informant Recruitment 231

Appendix D: Informed Consent Form 232 
Appendix E: Interview Protocol for Executive/Medical/Program Director 


\section{List of Tables}

$\begin{array}{ll}2.1 \text { Medicaid waiver programs } & 19\end{array}$

$\begin{array}{ll}2.2 \text { Policy overview } & 21\end{array}$

2.3 Typology of HCO performance 22

2.4 IOM ten rules for redesigning and improving health care 23

3.1 Definitions of core concepts of research questions 60

3.2 Operational definitions of context elements 61

3.3 Selection of criteria links to context elements 71

3.4 Site selection criteria $\quad 72$

3.5 Categories of interviewees $\quad 81$

3.6 Interview protocol question links to context elements 83

3.7 Qualitative analysis steps and definitions $\quad 84$

3.8 Relationship among context elements, institutional structure, and coding $\quad 85$

4.1 Policy-regulative element categories of support 107

4.2 Cultural-cognitive element categories of support 123

4.3 Normative element categories of support 136

4.4. Relational element categories of support 148

4.5 Procedural element categories of support 160

4.6 Institutional elements supports summary for IDTs 163

5.1 Institutional elements and supporting categories discussed in Chapter Four 165

5.2 Most significant findings for each element 195 


\section{List of Figures}

1.1 Institutional structure of health care in the U.S. and context elements

2.1 PACE program growth 1997-2013

3.1 Conceptual framework for research

3.2 Overview of research design logic 


\section{Chapter 1 Background}

Coming together is a beginning. Keeping together is progress. Working together is success. - Henry Ford, 1937

\section{Introduction}

The aging process will affect every person in a different way. Physical, cognitive, or social changes are all part of the biological process of aging; however, the speed at which those changes may occur is highly variable. What does not change is an individual's need for high quality, patient-centered care that will allow them to be healthy and independent as long as possible. As health care delivery continues to evolve from being professionally dominated to being patient-centered, the effect of the aging population will be a significant factor -- both in the sheer numbers of older adults collecting Medicare and the increasing longevity of older adults living in the United States. Health care organizations and the institutions that influence health care services are seeking to manage the spiraling health care costs that come with such growth, while continuing to improve the overall quality of care.

There are over 34 million Americans over the age of 65, a number that will double in less than thirty years (Jacobsen, 2011). Health care research and technology have been so successful in extending the lifespan that it has progressed to the point where a record number of Americans will be over the age of 85 and many of these individuals will require sophisticated levels of care management and coordination (Flaherty, 2004; Gawande, 2007). Older adults have high rates of chronic disease and disability; as 
longevity increases so will the need for more intensive care management (Crimmins, 2004; IOM, 2008). Eighty-two percent of older adults have at least one chronic disease that requires coordinated care and management (Pleis \& Lethbridge-Cejku, 2007; Pleis, Ward \& Lucas, 2010). What creates the next level of complexity beyond multiple chronic diseases is the natural breakdown of recovery caused by geriatric syndromes. Older adults may experience geriatric syndromes such as delirium, cognitive impairment, falls, sensory impairment, malnutrition, and osteoporosis, which make the diagnosis and prognosis determination complex and ongoing, instead of a simple curative regimen.

Aging and policy. An increase in the number of Americans over the age of 65, compounded by the number of chronic conditions experienced, on average, creates a significant public issue. Medicare is a federal, single-payer health insurance that pays for acute, sub-acute, some home care and hospice care. Medicaid is jointly funded by the federal and state governments and is administered and regulated by the states. The rates of disease and disability among older adults will continue to contribute to high rates of utilization of health care services and an increasing cost burden on Medicare and Medicaid. Medicare and Medicaid coverage make up 21 percent of the national budget (Center for Budget and Policy Priorities, 2012), which makes them the costliest portion of the nation's expenditures. Medicare beneficiaries with more than one condition visit eight physicians in a single year on average, and beneficiaries with five or more chronic conditions account for two-thirds of all Medicare spending (Anderson, 2003; IOM, 2008). The fate of these programs is unknown; it cannot be assumed that the programs will exist in perpetuity, as both Medicare and Medicaid are reported to be at the brink of 
exhaustion (Schulz, 2001; United States Board of Trustees of the Federal Hospital Insurance Trust, 2011).

A collaborative approach to care by an interdisciplinary team (IDT) has been shown to effectively manage long-term care costs and utilize the health workforce more efficiently (IOM, 2008). To help address the burden to Medicare and Medicaid, in 1997 the federal government introduced the Program for All-Inclusive Care of the Elderly (PACE) as a permanently recognized provider type. Unique characteristics of the PACE program include its capitated managed care benefit for frail elderly and the comprehensive medical and social service delivery system using an IDT. The IDT approach is housed within a local, adult day center, that is supplemented by in-home and referral services in accordance with the participants needs. PACE may be run by either public or private entities and must have the following characteristics:

- An IDT approach to providing care with prescribed members;

- A governing board that includes community representation;

- A physical site to provide day services;

- Defined service area;

- Safeguards against conflict of interest;

- Demonstrated fiscal soundness; and

- Have a formal Participant Bill of Rights (CMS, 2006).

The program is patient-centered, and the IDT is expected to include patients, family and health care representatives in health care decisions. In the legislation, states and health institutions are given flexibilities to meet the needs of their communities; the programs are not expected to be identical. 
PACE is included in health reform legislation, and is anticipated to be a model for long-term care services to the frail elderly. The Affordable Care Act of 2010 (Patient Protection and Affordable Care Act, 42 U.S.C. § 18001 (2010). is a comprehensive legislative effort to reform health care, and it will affect Medicare and Medicaid program administration and enforcement. The ACA is worth noting, but it is not the focus of this dissertation. The ACA is only one potential source of fundamental change in health care delivery and payment in the near future. At the state level, there is extensive and liberal use of Medicaid waiver programs that create state-level variations in care delivery.

Workforce issues (such as recruitment, retention, training, and quality) are significant throughout health care, and the growing aging population creates specific workforce challenges. Changes at the workforce level come in the form of a redefining of roles and responsibilities in order to increase efficiency and encourage individuals to work at the top of their license, allowing less expensive labor to take on more responsibility. Although the pace of evidence-based improvement is slow among health care institutions, one area where an urgent call for action has been made is in the care of older adults (Bragg \& Hansen, 2011; IOM, 2008). To address the issues of effective and efficient health care simultaneously, IDTs have been called for to be the primary care model for complex geriatric cases (IOM, 2008; JCAHO, 2006; Siegler, 1998).

Interdisciplinary teams caring for older adults. Many researchers have been advocating for the use of IDTs in the care of older adults due to the complexity of care needs and the rate at which older adults utilize health care resources (Flaherty, 2004; 
Heinemann, 2002; IOM, 2008; Siegler, 1998; Zeiss, 1996). External and internal elements in an institutional environment require the most efficient use of providers at all levels. However, there are numerous obstacles that make changing to a team-based care delivery model formidable, even for a well-managed health care organization.

Typically, IDTs are defined as consisting of individuals from at least two different disciplines who collaborate to deliver care to patients (Farrell, Schmitt, \& Heinemann, 2001). What distinguishes interdisciplinary from other team structures is the expectation that the team will engage in shared problem definition and problem solving processes. A single team member will make few decisions about a patient without consulting the team. In the most effective teams, team members pool their expertise so that patients receive better care, by collaborating so that the work can be done more effectively and efficiently (Drinka \& Clark, 2000). The benefit of collaboration will not only improve care but can assist in alleviating the effect of health workforce shortage issues.

Meeting the health care needs of the growing aging population through alternative workforce models has been the focus of many studies; of particular interest to researchers is the question of how the growing aging population will affect the workforce providing care for the elderly (Baxter \& Markle-Reid, 2009; Bragg \& Hansen, 2011, Miller, Booth, \& Mor, 2008; IOM, 2008; Stone, 2004). There are persistent recruitment and retention issues in the geriatric specialties of most disciplines (Coogle, Parham, Cotter, Welleford \& Netting, 2005; Miller, Booth \& Mohr, 2008; Stone, 2004; Warshaw\& Bragg, 2003), 
including medicine, nursing, allied health, and other health occupations (Bragg, Warshaw, Meganathan \& Brewer, 2010; Gawande, 2007; IOM 2008; Stone, 2004).

When dealing with complex clinical issues, teams provide efficiency, as a team can distribute the burden of treatment among the staff and offer a more diversified and objective perspective than a solitary staff member working alone. From the patient's point of view, teams can improve access to care and other resources; they may benefit from a variety of services and perspectives from multiple disciplines. However, not all patients or patient care situations require teams. While an effective team can quickly assess a situation, decide what professionals need to be involved, and work closely together to reach a solution, assigning a team to a project may not always be the most effective or efficient use of health care resources. Some tasks can be more efficient if assigned to just one person who has the knowledge, skill, and time to do the task independently (Cashman, Reidy, Cody \& Lemay, 2004; Lemieux-Charles \& McGuire, 2006; Reeves 2010; Wise, 1974). The appropriate use of teams requires an institutional and organizational commitment to support a truly interdisciplinary approach.

Variation in the decision to use a team-based model to provide care to a particular patient is dependent on the level of complexity of the clinical issues, the location in which the services are being provided, and organizational support for use of a team approach. Use of interdisciplinary teams continues to be endorsed by national organizations, including the American Geriatrics Society (Mion, Odegard, Resnick \& Segal-Galan, 2006) and accrediting bodies such as the Joint Commission on 
Accreditation of Healthcare Organizations (JCAHO, 2006). JCAHO requires evidence of interdisciplinary collaboration in hospitals, medical homes, nursing homes and outpatient settings as part of its accreditation process (Dellefield, 2006; JCAHO, 2011a; JCAHO, 2011b; JCAHO, 2011c; Kohn, Corrigan, \& Donaldson, 2000). The Institute of Medicine (IOM) has repeatedly called for an interdisciplinary approach to care for older adults in most long-term care environments (1996; 2001; 2008; 2009).

In order for a widespread, consistent utilization of teams in the care of older adults, a level of institutional legitimacy is required. Merely having appropriate resources and best-practice designation is not enough. If teams are to survive and thrive within a health care institution, they will need a high level of acceptability and credibility (Scott, 2000) known as legitimacy. Legitimacy is a generalized perception or assumption that the actions of an organization are desirable and appropriate within an institutional framework of norms, beliefs, values, and definitions (Suchman, 1995; Scott, 2008a). Legitimacy is not a product or service that can be owned or exchanged, but a condition that reflects an understood collaboration within the relevant cultural-cognitive, normative, and regulative frameworks. It is largely symbolic in value, but it does have great power within health care organizations to rearrange established models of care delivery and create opportunities that would otherwise be impossible to fashion. In order to create such legitimacy, the presence of the appropriate institutional context is essential.

Institutional Context. The members of a given interdisciplinary team are often simultaneously members of a particular health service organization. This "home" 
organization defines the team, its members, tasks, and the time and place of team interaction. The health services organization is nested within a larger institutional structure that regulates funds, legitimizes, and possibly provides goals and objectives for the organization. Context is a social-psychological construct of the environment that exists at multiple levels of the organization or governing institution (Chafetz, West \& Ebbs, 1987). Thus, institutional context is important because of its broader setting, the organization's goals, structure, locus of authority and control - all of which markedly influence how the team operates. Context is also influenced by the norms and values of the organization, which will play a role in the ability of an IDT to do its job. Organizational characteristics will develop within and respond to a particular context, created by formal and informal structures that require attention and resources. Scott (2000) and Reeves (2010) provided frameworks of institutional analysis based on five elements that play a role in the context of health care organizations and the teams that work in them. The five institutional elements include cultural-cognitive, normative, policy-regulative, relational, and procedural structures. By drawing attention to each of these five elements, organizations will be framed as technical and production systems responding to resource availability. They will also be recognized as social systems influenced by cultural rules, normative, relationships and legal frameworks. Figure 1 provides the structural layers of health care organizations and delineates which layers are influenced by each of the five elements (Reeves; 2010; Scott, 2008a).

Institutions that govern health care organizations define the context that can develop and sustain preferred care delivery structures. These institutions can influence 
behavior at all levels of care delivery from the Certified Nursing Assistant (CNA) who moves a patient regularly to avoid bedsores, to the Chief Financial Officer (CFO) who decided the budget allotment for the program that pays the CNA. The institutions and health care organizations specify the rules, governance structures, and meanings that inform the decisions made by the administrators and providers alike. In general, institutions are the ground that supports and defines the activities of the organization.

Figure 1.1 Institutional Structure of Health Care in the U.S. and Context Elements

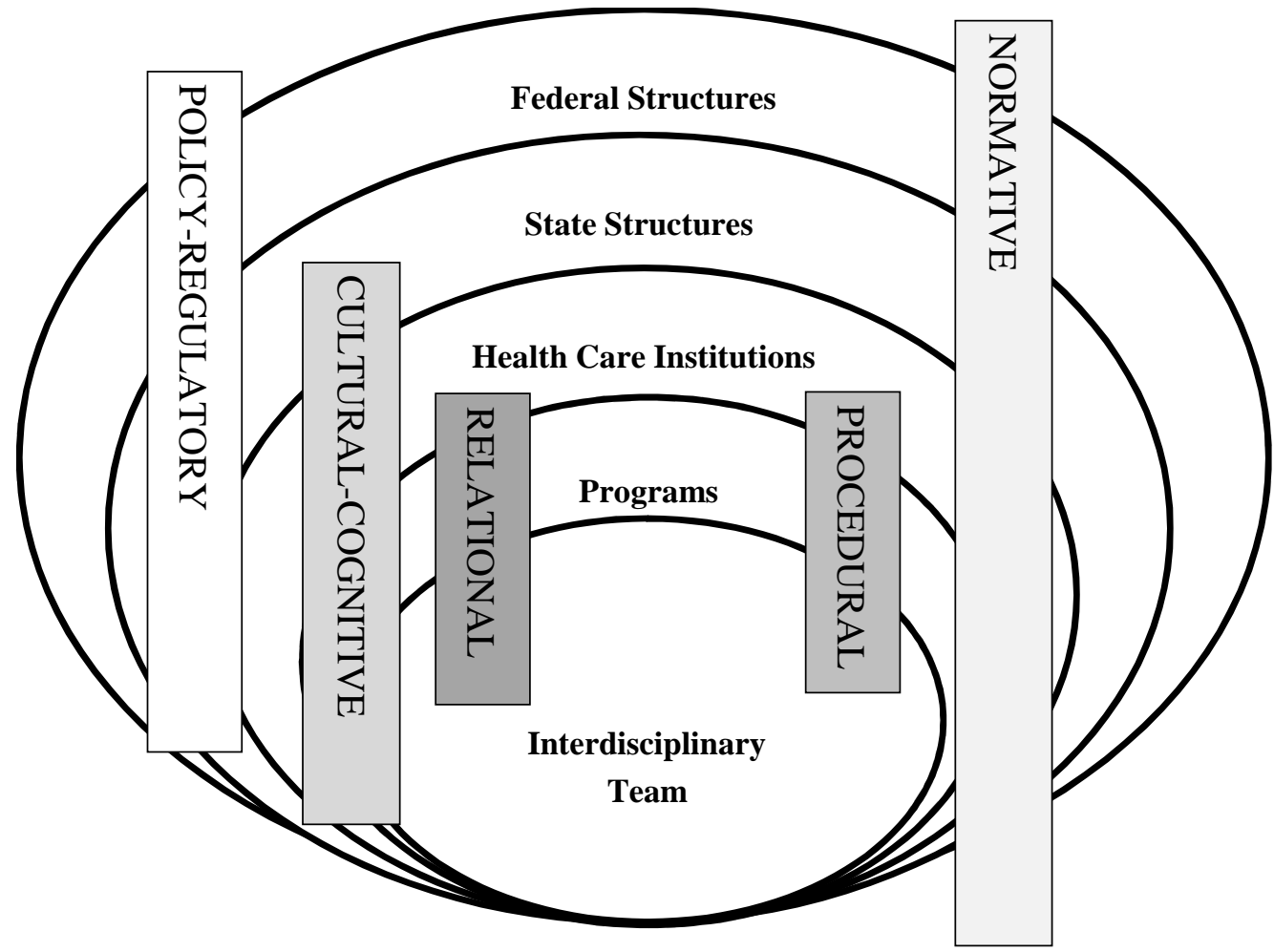

Examining health care organizations and their governing institutions in the United

States is a unique experience. Many nations organize and deliver health care within a single-payer, nationally run system. In the U.S., our delivery mechanisms are closely tied 
to market structures. Researchers have noted (Abbott, 1988; Crimmins, 2004; Dellefield, 2006; Shortell, 1985) significant changes in the delivery of care since the 1960's, which marked the initial shift from a health care system dominated by one entrenched profession to a more diversified field of service delivery models, professions, and payment possibilities. Change is normal for health care organizations and with an everincreasing focus on improvement and monitoring of outcomes (Bisognano \& Kenney, 2012) and the enactment of the ACA passed in 2010; significant institutional level change is anticipated. Persistent change creates uncertainty in the environment; depending on the institutional philosophy, responses and adaptations will vary.

There is a divide between theory and practice, and between health care organizations and the use of teams. Researchers, academics, philanthropic foundations and practitioners advocate the use and implementation of structures to support team care in a diverse array of settings. As health professions are training their students to practice in interdisciplinary teams, and health reforms are restructuring the way care is provided in the U.S., understanding the influence of the institutional context will contribute to sustainability of interdisciplinary team models of care. These calls for team practice have been heard for many years from the geriatric care community. Even with successful team-based models gaining legitimacy, adoption of best practices has been very slow to become everyday practice within health care institutions. Balas, Boren \& Brown (2000) found that it could take over seventeen years for an established best practice to become common practice. Today, interdisciplinary care models are legitimate practice in nursing homes, primary care, and hospice care settings, yet they are far from common practice. 


\section{Problem Statement}

There is a robust literature on team effectiveness in the care of older adults (Bruce, 1980; Drinka \& Clark, 2000; Jelphs \& Dickinson, 2008; Katzenbach \& Smith, 1994; Lemieux-Charles \& McGuire, 2006; Reeves, 2010). Nonetheless, there is a substantive gap in the understanding of how health care organizations and their institutions might influence the organizational legitimization of teams as a care delivery model. Two significant steps towards legitimization of teams as a care delivery model have occurred in recent years. Interdisciplinary work is now included as a part of accreditation through the JCAHO (JCAHO, 2011a). Another major milestone was the formalization of capitated payment (IOM, 2008) for the Program for All-Inclusive Care of the Elderly (PACE), through a coordinated Medicare/Medicaid funding stream. There have been many other innovations and attempts to legitimize interdisciplinary team care for the elderly, but few models have proliferated across the U.S (Boult \& Weiland, 2010; Cooper \& Fishman, 2003; Counsell, Callahan, Tu, Stump \& Arling, 2009; Inoyue, Bogardus, Baker, Leo-Summers \& Cooney, 2000; IOM, 2008).

The complex nature of caring for the older adult benefits from the contributions of multiple disciplines in care planning. Indeed, the value of teamwork is intuitively appealing (Bosch, Dijkstra, Wensing, Vander Weijden \& Grol, 2008; Rafferty, Ball \& Aiken, 2001; Reeves, 2010). However, there are issues in legitimizing the use of teams in the care for older adults on a broad scale. Teams are personnel intensive and require new ways of working and learning for professionals at all levels. Organizational hiring practices and provider training affect the ability of teams to collaborate coordinate, and 
network, all of which play a part in legitimizing and supporting teams at every level of a health care organization (Reeves 2010). There is currently great variation in the utilization of interdisciplinary teams in the care of older adults. Teams may be called interdisciplinary, but there is little consistency in how that idea is interpreted and put into practice.

The structure of an organization and its institutions creates the environment where teams work. Institutional and organizational reorganization is resource intensive. A supportive institutional culture is vital to optimal team utilization and effectiveness in providing high-quality care and positive patient outcomes (Heinemann \& Zeiss, 2002). The organization's ability to make structural space for team-based care models are dictated by broader institutional effects that can help or hinder the adaptation and use of teams.

\section{Research Question}

The question to be pursued during the course of this dissertation is:

What elements of institutional context support the use of interdisciplinary teams in the care of older adults?

\section{Purpose of Study}

The purpose of this study is to explore the institutional variation in the utilization and legitimacy of interdisciplinary teams in the care for older adults. Given the complicated and multi-faceted nature of health care organizations and their institutions in the U.S., this study will be a first step in understanding how teams are supported and 
thrive in organizations that have committed to using a team-based model. This study will examine the relationship between institutional elements and the utilization of interdisciplinary teams, and explore the extent to which institutional decision-makers consider team-based models in policy and procedure changes in the delivery of care.

\section{Significance of Study}

This dissertation will explore the nature of institutions to support new care delivery models, create the structural space, and provide other resource supports necessary for those new models to thrive. Brallier and Tsukuda (2002) strongly connect organizations and institutional support as a critical piece of effectively providing and sustaining team-based care. Identifying facilitators and obstacles in the legitimization process allows the emergence and adoption of team models, and assists in understanding the contextual influence observed across health care organizations and their governing institutions. An individual provider's perception and preparation to incorporate change in practice are significant and have been studied at length (Bellman, Webster \& Jeanes, 2011; Herzlinger, 2004; Liao \& Ackermann, 2008; Mahoney et al., 2010; Stock, Reece \& Cesario, 2004). The PACE programs selected as the cohort for this study are required to support an IDT to provide care to patients. Examining the contexts that each program functions under will illuminate what elements are important in sustaining the team model and thus the program.

Identifying individual and organizational limitations within a health care institution will illuminate how internal and external structures will need to evolve. 
Institutional support and reorganization can be a slow process and initially it may be difficult for decision makers to justify how to make the initial investment worthwhile. The results of this study will be used in a first step to understanding how an organization can integrate team-based care and what it means for the broad spectrum of stakeholders for the health care organization and its institutional context.

\section{Conclusion}

It is increasingly important for health care organizations, providers, and patients to understand the continuum of influence on health care delivery decisions. This study will facilitate the understanding of how institutions influence choices made around care delivery models and add to the literature in organizational theory, health administration, and workforce policy and practice. 


\section{Chapter 2 Review of the Related Literature}

\section{Introduction}

This chapter reviews the relevant areas of policy and literature that provide the foundation for this dissertation. This chapter includes:

1) A discussion of the relevant policy context that is important to care provision for older adults and the evolution of the Program for All-Inclusive Care of the Elderly (PACE), including Social Security, Medicare, Medicaid, Balanced Budget Act of 1997, the Affordable Care Act of 2010, and a brief discussion of the quality movement;

2) An examination of organizational institutionalism including a brief discussion of core concepts, application to institutional context and IDTs, and a description of Scott's institutional framework;

3) An overview of the theory of professionalization and the role of professions in institutionalism;

4) A brief history of teams in health care, along with a review of the theoretical considerations for teams in health care settings and how teams have been used in geriatric care;

5) Finally, a brief overview of the Program for All-Inclusive Care of the Elderly (PACE) including a program history, regulation and policy, financing and barriers to growth. 


\section{Health and Social Policy Context}

We can never insure one hundred percent of the population against one hundred percent of the hazards and vicissitudes of life, but we have tried to frame a law which gives some measure of protection to the average citizen, and his family against the loss of a job and against the poverty-ridden old-age. - Franklin Delano Roosevelt, at the signing of the Social Security Act, August 14, 1935

Current federal level policies play and important part in defining the institutional

context. Older adults receive entitlement benefits that give the government a special interest in the delivery of health care services to older adults, and in the quality of those services. This section will outline how federal policy has influenced health and social service delivery to older adults through Social Security, Medicare, Medicaid, the Balanced Budget Act of 1997 and the Affordable Care Act (ACA). There will be a brief discussion of the quality movement as well and its role in influencing many current policy decisions.

Social Security. Any discussion of federal policy and health care for older adults must start with the Social Security Act (SSA) of 1935. Prior to the SSA, care of the elderly was solely the responsibility of families and communities. The SSA intends to provide a safety net for older and disabled Americans as they reach retirement age or when they reach a point where they can no longer work (Schulz, 2001). The safety net created by the SSA aims to protect the most vulnerable Americans from living in abject poverty. As ambitious as the SSA was, its lack of a health care provision was a glaring omission. That omission was remedied in 1965 with the creation of Medicare and Medicaid. 
Medicare. Medicare is an entitlement program that one can become eligible for if they or a spouse works ten years in Medicare covered employment, are 65 or older, and a citizen or permanent resident of the United States (CMS, 2011). Many economists are reporting that the Medicare trust will be "exhausted" as of 2029 (Social Security Administration, 2010), a direct result of having too many beneficiaries and too few taxpayers. Many Americans incorrectly assume Medicare will provide full-coverage of their health and long-term care needs. Medicare is the primary payer of primary care and chronic disease management; however, it only covers nursing home care only when a beneficiary requires daily skilled inpatient nursing or rehabilitation services after a hospital stay (Schulz, 2001; Wunderlich \& Kohler, 2001).

One of the earliest innovations in team reimbursement structures was the formalizing of the hospice benefit under Medicare in 1986. Hospice manages end-of-life care for patients who have a prognosis of six months or less. Hospice is reimbursed by the case and not by the visit (Vogt, 2006). A team is assigned to each case and consists of a doctor, nurse, chaplain (or other counselor), social worker, and trained volunteers. From 1999-2007 Medicare hospice users grew by 110 percent while spending on hospice grew by 229 percent (Ng, Harrington, \& Kitchener, 2010). Hospice is a highly valuable team-provided care model for the individuals receiving care and their families (Higginson et al., 2003; Lorenz \& Agency for Healthcare Research and Quality, 2004; Morrison \& Meier, 2004). Medicare hospice benefits improved quality of care at the end of life, increased satisfaction with care and reduced the cost to Medicare, which is not a common confluence of events. 
Medicaid. Medicaid is jointly funded by federal and state governments. Each state administers its own version of the Medicaid program while the federal Centers for Medicare and Medicaid Services (CMS) monitor the programs. Medicaid is a meanstested program, and not an entitlement like Medicare. Medicaid pays the health care costs for older adults who live at the poverty line or have depleted their assets enough to qualify. Medicaid pays for 42 percent of all nursing home and home health care expenditures (Hartman, Martin, McDonnell, \& Catlin, 2009). Since Medicaid is administered at both the federal and state level, it provides a unique set of administrative challenges. Each state sets their own payment level, establishes their own eligibility criteria, and defines what services are covered; many of those services are subject to broad federal guidelines. These federal guidelines require the state to cover nursing facility care for individuals over 20 years of age and home health services for those eligible for skilled nursing care (Wunderlich \& Kohler, 2001). One area of Medicaid policy that is particularly relevant to interdisciplinary teams (IDTs) is the waiver program to innovate and integrate new models of care delivery at the state level. These waiver programs allow the state authorities greater discretion in how funds are spent and what populations are targeted. Table 2.1 differentiates the three primary Medicaid waiver programs: Sections 1115, 1915(b), and 1915(c).

All three of the Medicaid waiver programs provide discretion to the states to make decisions not just about service delivery but also who delivers those services and what they get paid. Long-term care (LTC) provided in institutional settings accounts for a major portion of the overall Medicaid expenditures. LTC accounted for 40 percent of 
national Medicaid expenditures, but the percentage varied greatly from less than 30 percent for some states to nearly 60 percent for others (Wunderlich \& Kohler, 2001). This level of variation adds to the overall complexity and availability of public program services within and across state lines (Ng, Harrington, \& Kitchener, 2010).

Table 2.1: Medicaid Waiver Programs

\begin{tabular}{|c|c|c|c|c|}
\hline $\begin{array}{l}\text { Waiver } \\
\text { Section }\end{array}$ & Intent & Limitations & Evaluation & Population \\
\hline $\begin{array}{l}\text { Section } 1115 \\
\text { Research \& } \\
\text { Demonstration } \\
\text { Project }\end{array}$ & $\begin{array}{l}\text { Create upstream } \\
\text { policy learning, from } \\
\text { the state to the } \\
\text { federal government } \\
\text { May provide new } \\
\text { services or use } \\
\text { utilized new } \\
\text { approaches to care, } \\
\text { including non- } \\
\text { traditional workforce } \\
\text { skill mixes and } \\
\text { payment structures }\end{array}$ & $\begin{array}{l}\text { Any pilot } \\
\text { demonstration } \\
\text { program must } \\
\text { promote the } \\
\text { objectives of } \\
\text { Medicaid }\end{array}$ & Annually & $\begin{array}{l}\text { May recruit } \\
\text { individuals who } \\
\text { are not } \\
\text { Medicaid } \\
\text { beneficiaries }\end{array}$ \\
\hline $\begin{array}{l}\text { Section } \\
1915(\mathrm{~b}) \\
\text { Managed } \\
\text { Care/Freedom } \\
\text { of Choice } \\
\text { Waivers }\end{array}$ & $\begin{array}{l}\text { Focus on alternative } \\
\text { delivery and } \\
\text { payment systems } \\
\text { Restricts the } \\
\text { "freedom of choice" } \\
\text { and restrict } \\
\text { beneficiaries' right } \\
\text { to choose their own } \\
\text { providers }\end{array}$ & $\begin{array}{l}\text { Must } \\
\text { demonstrate } \\
\text { cost } \\
\text { effectiveness } \\
\text { and efficiencies } \\
\text { Five year limit }\end{array}$ & $\begin{array}{l}\text { Not } \\
\text { required }\end{array}$ & $\begin{array}{l}\text { Must be } \\
\text { Medicaid } \\
\text { eligible }\end{array}$ \\
\hline $\begin{array}{l}\text { Section } \\
1915(c) \text { Home } \\
\text { and } \\
\text { Community- } \\
\text { Based } \\
\text { Services } \\
\text { Waivers }\end{array}$ & $\begin{array}{l}\text { Allows provision of } \\
\text { long-term care } \\
\text { services in home and } \\
\text { community based } \\
\text { settings. }\end{array}$ & $\begin{array}{l}\text { Enrollees must } \\
\text { qualify for long- } \\
\text { term care in an } \\
\text { institutional } \\
\text { setting }\end{array}$ & $\begin{array}{l}\text { Audit or } \\
\text { review } \\
\text { upon } \\
\text { request }\end{array}$ & $\begin{array}{l}\text { Disabled and } \\
\text { Medicaid } \\
\text { eligible }\end{array}$ \\
\hline
\end{tabular}


The Balanced Budget Act of 1997. The Balanced Budget Act (BBA) of 1997 established the Program for All-Inclusive Care of the Elderly (PACE) model as a permanently recognized provider type under both the Medicare and Medicaid programs (CMS, 2006). It defines PACE, requirements for providers, payment structures, and trial periods under federal law. It also outlines the states' responsibilities in the support and monitoring of the programs. Currently there are 96 PACE programs in 31 states (National PACE Association, 2013).

The Affordable Care Act of 2010. The Affordable Care Act of 2010 (ACA) directs the Surgeon General to establish programs in continuing medical education for health professionals that emphasizes patient-centered, interdisciplinary, and care coordination skills, throughout the continuum of health related education, training, and practice (Goodson, 2010). Of great significance to the providers, ACA also gives an automatic 10 percent increase in Medicare payments for primary care provided by a physician, nurse practitioner, or physician's assistant who receives at least 60 percent of their income from providing primary care. ACA, if it remains intact as passed in its entirety, can fundamentally change the training and practice of our health care workforce. It will allow for an increase in the number and quality of individuals who are trained in geriatrics to care for older adults. Medicare, Medicaid and the ACA have the potential to address many of the institutional obstacles to adaptation and legitimization of IDTs in the care of older adults. Table 2.2 summarizes the primary contribution of the policies described in this section. 
The quality movement. Health care organizations ( $\mathrm{HCO})$ would once strive to provide the highest quality care to all patients without consideration of cost (Shortell, 1985). No longer can unbridled quality be pursued without serious consideration of the Triple Aim (Berwick et al., 2008) or quality, cost, and access. In attempts to meet the Triple Aim, health policy researchers and administrators are embracing transformation in order to align all health care with the Triple Aim. This is particularly pertinent for publicly funded health care from Medicare and Medicaid, for example. Many of the incentives for promotion of interdisciplinary care arose out of financial pressures to reduce costs, workforce shortages, and improving quality. Ideas around increasing productivity that were first applied to industry and manufacturing began to make its way to health care delivery (Harrington \& Estes, 2008).

Table 2.2: Policy Overview

\begin{tabular}{|c|c|c|}
\hline Policy & $\begin{array}{l}\text { Signed into Law } \\
\text { (Date/By) }\end{array}$ & Contribution to Care of Older Adults \\
\hline $\begin{array}{l}\text { Social } \\
\text { Security }\end{array}$ & $\begin{array}{l}\text { August 14, } 1935 \\
\text { Franklin Delano } \\
\text { Roosevelt }\end{array}$ & $\begin{array}{l}\text { - Constructed the federal infrastructure for } \\
\text { social safety net }\end{array}$ \\
\hline Medicare & $\begin{array}{l}\text { July 30, } 1965 \\
\text { Lyndon Baines Johnson }\end{array}$ & $\begin{array}{l}\text { - Comprehensive health coverage for older } \\
\text { Americans }\end{array}$ \\
\hline Medicaid & $\begin{array}{l}\text { July 30, } 1965 \\
\text { Lyndon Baines Johnson }\end{array}$ & $\begin{array}{l}\text { - Comprehensive coverage for poor, } \\
\text { disabled, and elderly }\end{array}$ \\
\hline $\begin{array}{l}\text { Balanced } \\
\text { Budget Act }\end{array}$ & $\begin{array}{l}\text { August 5, } 1997 \\
\text { William Jefferson Clinton }\end{array}$ & $\begin{array}{l}\text { - Created PACE payment structure and } \\
\text { payment systems }\end{array}$ \\
\hline $\begin{array}{l}\text { Affordable } \\
\text { Care Act }\end{array}$ & $\begin{array}{l}\text { March 23, } 2010 \\
\text { Barack Hussein Obama }\end{array}$ & $\begin{array}{l}\text { - Legislates pay increases for provider with } \\
\text { over } 60 \% \text { Medicare/Medicaid } \\
\text { beneficiaries } \\
\text { - Mandates greater IDT training }\end{array}$ \\
\hline
\end{tabular}


Quality initiatives are now found throughout all health and social service organizations, including where IDTs are working and redefining the role of leadership and engaging patients (and family) into some team models (Siegler, 1998; Xyrichis \& Lowton, 2008; Zeiss \& Steffen, 1996). Table 2.3 is a summary of Shortell's typology of HCO performance, based on willingness and ability of HCO's to improve performance. In quadrants of mediocre performance, the focus area for improvement is highlighted.

Table 2.3: Typology of HCO performance

\begin{tabular}{|c|c|c|c|}
\hline & \multicolumn{2}{|c|}{ Ability } \\
\hline \multirow{3}{*}{$\underset{\infty}{\infty}$} & & YES & $\mathrm{NO}$ \\
\hline & YES & $\begin{array}{l}\text { - High performing health care } \\
\text { organization }\end{array}$ & $\begin{array}{l}\text { - Mediocre performance in need of } \\
\text { talent and skill development } \\
\text { - Requires adequate workforce } \\
\text { and resources }\end{array}$ \\
\hline & $\mathrm{NO}$ & $\begin{array}{l}\text { - Mediocre performance in } \\
\text { need of discernment and } \\
\text { courage } \\
\text { - Requires institutional and } \\
\text { organizational willingness }\end{array}$ & $\begin{array}{l}\text { - Low performing health care } \\
\text { organization }\end{array}$ \\
\hline
\end{tabular}

Satisfaction, quality, and efficiency became the center of the quality movement when the IOM published Crossing the Quality Chasm (2001), which reported that not only "health care quality was not where it should be", but that the current system did not have the capacity to make it what it needs to be (Berwick, 2002, p.83). The IOM Committee proposed six aims for improvement: safety, effectiveness, patientcenteredness, timeliness, efficiency, and equity (IOM, 2001). To assist with the operationalization of these concepts the IOM proposed 10 corresponding rules to further 
illustrate the differences between what the current dysfunctional system does and what it should aspire to; these rules are summarized in Table 2.4.

In order to see the type of improvement that the IOM report was calling for, a definition of aims would be necessary. Berwick, Nolan, and Whittington (2008) proposed the Triple Aim as the focus for improving a beleaguered U.S. health system. The three directives` of the Triple Aim are:

1. Improve the individual experience of care

2. Improve the health of the population

3. Reduce per capita health care costs.

Table 2.4: IOM ten rules for redesigning and improving health care

\begin{tabular}{|c|l|l|}
\hline & \multicolumn{1}{|c|}{ State of System (circa 2006) } & \multicolumn{1}{c|}{ Aspiration for System } \\
\hline 1. & Care based primarily on visits & $\begin{array}{l}\text { Care based on continuous healing } \\
\text { relationships }\end{array}$ \\
\hline 2. & $\begin{array}{l}\text { Professional astronomy drives } \\
\text { variability }\end{array}$ & $\begin{array}{l}\text { Care is customized according to patient } \\
\text { needs }\end{array}$ \\
\hline 3. & Professional control care & Patient of the source of control \\
\hline 4. & Information is a record & Knowledge is shared freely \\
\hline 5. & $\begin{array}{l}\text { Decision-making is based on training } \\
\text { and experience }\end{array}$ & Decision making is based on evidence \\
\hline 6. & $\begin{array}{l}\text { "Do no harm" is an individual } \\
\text { responsibility }\end{array}$ & Safety is a system property \\
\hline 7. & Secrecy is necessary & Transparency is necessary \\
\hline 8. & System needs to react to patient needs & Patient needs are anticipated \\
\hline 9. & Cost reduction is sought & Waste is continuously decreased \\
\hline 10. & $\begin{array}{l}\text { Preference is given to professional } \\
\text { roles over the system }\end{array}$ & Cooperation among clinicians is a priority \\
\hline \multicolumn{2}{|l|}{ Adapted from IOM 2001 }
\end{tabular}

For many health care organizations, this improvement can be best achieved for older adults, in part, through IDTs. The recommendations to enhance quality have 
focused the many policy decisions around the "iron triangle" of health care of quality, access, and cost, which is a significant contribution of the quality movement to the health policy agenda (Wenzel \& Rohrer, 1994). In order to understand the formulation and implementation of policies discussed in this section, the next section will discuss organizational institutionalism and its role in influencing behaviors of the many actors in the policy process.

\section{Organizational Institutionalism}

"In the past couple of decades institutional theory has predominantly provided insights into processes that explain institutional stability rather than change, and the emphasis has been on how institutional pressures force organizations to converge on a standard set of practices . . . There has been little attention toward unpacking processes of how institutions are socially constructed. This question becomes crucial . . in regard to how new institutions are created and existing ones changed." - Phillips \& Malhotra, 2008

Institutions are defined as social or organizational behavior that is facilitated by normative, cultural, a nd regulative systems that provide ones understanding of social exchanges and the reproduction of desired actions or decisions (Greenwood, 2008). Institutional studies are interdisciplinary in nature with influences from across the social sciences spectrum. This section will discuss many of the terms fo reference in order to provide a foundation for use of an neo-insitutional approach in this study. The final section will discuss institutional context and its established relationship to health care teams.

Neo-institutional theory core concepts. The power of institutional influences are not always obvious, but organizations must negotiate with these deep rooted forces. 
Not all organizations are institutions, but most organizations must contend with some institutional forces, which may be formal or informal in structure. Meyer and Rowan (1991) defined institutional context as the rules, norms, and ideologies of wider society. This context will lead organizations to deal with their environments at their boundaries and imitate their environment in their structures. Prescriptions of what is considered suitable behavior and conduct in and among organizations are also called rationalized myths. Meyer and Rowan (1977) credit two conditions in the diffusion of formal rules and structures through society: there must be a complex network of social organizations and exchanges, and the presence of appropriate contextual factors (Meyer \& Rowan, 1977). Resource dependency theory (RDT), an antecedent to Meyer and Rowan's work, examines organizations and their external environment. Resource dependency theory defines this relationship between an organization and its environment as a necessary reaction to properly distributing resources within an organization (Greenwood, 2008; Pfeffer \& Salancik, 1978). Institutions embody the understanding that rationalized myths define what it means to act rationally; sometimes this includes contradicting market or bureaucratic pressures that otherwise would influence organizational behavior.

Organizational institution (OI) theorists assume that as norms, culture, regulative structures are part of the social fabric then they become institutionalized and embody organizational practices (Deephouse \& Suchman, 2008; DiMaggio \& Powell, 1983; Zucker, 1983). The manifestation of social norms, culture, and regulative structures are integral in how organizations appear comparable or divergent. Isomorphism is an indicator of institutionalization as similar organizational forms and structures are believed 
to originate from several organizations complying with identical rationalized myths (DiMaggio \& Powell, 1983; Meyer \& Rowan, 1977). The diffusion of ideas and practices that become institutionalized is thought to result in isomorphism. DiMaggio and Powell (1983) outlined three primary mechanisms of institutional diffusion. Political influence and legitimization issues led to coercive isomorphism, uncertainty led to mimetic isomorphism, and professionalization led to normative isomorphism. The different origins of influences create various manifestations of isomorphism that can be observed in different types of institutionalization.

Organizational survival can depend highly on the appearance of conformance as it discourages closer examination of practices and may prevent the organization from being accused of deceitful activities (Boxenbaum \& Jonsson, 2008). Reservations regarding organizational efficacy can affect their legitimacy in their respective field. Definitions of legitimacy stem from normative, cultural, and regulative support for a particular practice or organization. Legitimacy can be very difficult to define concretely it can be easier to understand what legitimacy is not. Legitimacy is not a product or service to be purchased. Legitimacy is a level of normative, cultural, and political alignment within an organization and between organizations and their environments (Scott, 2008a). The actions of an organization are assumed desirable and appropriate within the systems of norms, values, beliefs and meanings (Deephouse \& Suchman, 2008).

Institutional logics are a set of beliefs and practices in an organizations' environment. Organizations may adapt their behavior to mimic its institutional 
environment. However, several institutional constructs have incompatible logics. Actors within organizations may find themselves perplexed about behavioral expectation and this will create a tension point between the conflicting logics. This conflict creates space for change that allows for the creation of new logics and behavioral expectations (Friedland \& Alfred, 1991).

DiMaggio and Powell (1983) and Meyer and Scott (1992) identified the unit of analysis for studying institutional processes as the "organizational field." Scott (2008a) defines an organizational field as "a community of organizations that partakes of a common meaning system and whose participants interact more frequently and fatefully with one another than with actors outside the field" (p. 56). The organizational field is the unit of analysis for studying the development and subsequent dissemination of institutional logics (Scott, 2008a). Since the initiation of organizational field as a recognized unit of analysis, it has been an accepted term of reference for neo-institutional theory and research (Pfeffer \& Salancik, 1978; Wooten \& Hoffman, 2008). The concepts of organizational institutionalism are critical to understanding how the environments of organizations may facilitate or create barriers to practice. Health organizations today have demonstrated reluctance to adapting new care delivery models, which may reflect the complexity of the institutional context that governs practice.

\section{Organizational and institutional context in supporting health care teams.}

The dominant organizational pattern in western society is bureaucratic, including health organizations, which allows these organizations to be hierarchical with power and 
authority concentrated at "higher levels" of the organization (Chaftez, West \& Ebbs, 1987). The IDT is a departure from a strict hierarchical model. Most teams are located within a hierarchical institution, and thus the management and support of the team are consistently affected by decisions made outside of the team.

The institutional elements that affect teams are represented in the literature through discussions around professional representation and institutional support. Professional representation comes in two forms in many health occupations: professional associations and unions. Professional associations have been calling for a more interdisciplinary approach to education and practice. These associations can influence health policy with their endorsement of a particular model of delivery (Greenwood, Suddaby \& Hinings, 2002). The endorsement can provide greater governmental and organizational support and funding. However, it is difficult to link the endorsement to actual policy decisions, including how teams are implemented and evaluated. Unions are a central piece of contract bargaining especially for nursing professions (Seago \& Ash, 2002). Unions can influence a workplace in many ways to create a more accommodating atmosphere for team-based care, including reducing discrimination and harassment in the workplace and providing a venue for reporting poor behavior without fear of retaliation (2002).

Institutional support plays a key piece in team function (Xyrichis \& Lowton, 2008). The rise of care management and health administration has resulted in a shift in the power balance from the professions to managing institutions (Ferlie, 1996; Scott, 
2000). Given that teams are embedded in management organizations, support from those organizations and their respective institutions are crucial (Baxter \& Markel-Reid, 2009; Onyett \& Campling, 2003). Organizational and institutional support ensures enough time and money to engage in collaborative care to respond to the needs of the patients most effectively. Team performance can diminish over time if there is a lack of organizational rewards for improvements in working practices (Cashmen, Rudy, Cody \& Lemay, 2004). Institutional support can encourage the use of innovations and facilitate implementation within teams (Dieleman et al., 2004), and facilitate improvements in quality of teamwork as a result of updating practice (Rutherford \& MacArthur, 2004). IDTs can experience complications in their relationship to their host organization. Team members can come to identify themselves as a team member over their connection and place within the organization. Dissociation at any level can affect the organization's political will to support team functioning, and any lack of support for the team can lead to feelings of apathy towards both work and the organization (Jelphs \& Dickenson, 2008).

Ducanis and Golin (1979) suggest five areas where the organization must support an IDT; if these supports are deficient then obstacles may arise that would interfere with team functioning. The first area is the philosophy of the institution and to what extent it supports interdisciplinary coordination. Supporting interdisciplinary coordination includes rewards and benefits systems, as well as personnel evaluation processes that reflect an interdisciplinary approach. The second area is the adequacy of the institution's staffing commitment. Institutions should protect team members' time, and not persistently require them to be involved with responsibilities outside of the team. The 
third area of support is physical space, and the extent to which teams are in competition for physical resources. Teams require adequate access to meeting and office spaces, proximity to other members, shared technology, disciplinary diversity, and adequate budgeting for team activities. The fourth requirement is the procedural boundary, which will protect the teams' time and operations. Boundary issues include team membership, meeting attendance, client load, and information access. The final requirement is the extent to which the organization reorganizes and supports the team authority and expertise in its skill area. The more legitimized the authority and expertise of a team within an organization, the less likely clinical judgments will be changed for non-clinical reasons. However, organizations are part of a larger institutional environment where their behavior may be constrained or enabled by external bodies.

Institutional support for health care organizations. Institutional environments influence organizations in several ways. Institutions provide an archetype for actors, legitimization of institutional logic, governance systems, and rules for social interaction (Alford \& Friedland, 1985; Scott, 2008a). Institutions also define the resources available to the organization, and how those resources can be used, combined, and operationalized. As much as institutions may influence behavior of individuals and organizations, individuals and organizations may also modify and influence their institutional environments. However, once the environment is constructed (even if internally created) it acts as an external force, which may facilitate or obstruct the individuals and organization in relation to particular behaviors. Institutions consist of cultural-cognitive, normative, and policy-regulatory structures and activities that provide stability and 
meaning to collective actions (Scott, 2008). The elements are not mutually exclusive, but they each come from a unique cultural context, the sum of which provides a comprehensive understanding of institutional influence (Greenwood, 2008; Meyer \& Rowan, 1977; Scott, 2008a).

Cultural-cognitive elements. Cultural-cognitive elements of institutions are shared conceptions that constitute the nature and social reality and the frames through which meaning is actualized (Scott, 2008a). The cognitive elements of human existence are of paramount importance, where it is necessary to mediate between the external environment and the response of an individual to that environment which creates symbolic representations of that environment (D'Andrade, 1984). Meanings arise through interaction and are maintained and transformed as they are used to make sense of what is going on. To create understanding of a situation, one must take into account the empirical conditions as well as the actor's subjective interpretation of those conditions. Scott (2008a) hyphenates "cultural-cognitive" as a singular element acknowledging that the internal interpretation processes are influenced by the external cultural frameworks.

Cultural elements vary in their degree of institutionalization. People in the same situation can perceive that situation very differently, in terms of both empirical observation and normative beliefs. Cultural patterns and behaviors occur due to a level of compliance that recurs in various circumstances; the behaviors are so routinized that is difficult for an individual to conceive of another way to act. Institutions often can provide the prefabricated organizing models, rules, and responsibilities. A particular 
belief system or cultural frame that administrators and providers adopt in decisionmaking may be imposed on frontline service provision (DiMaggio \& Powell, 1983).

Normative elements. At the core of the normative element of institutions is the emphasis on normative rules. Normative rules introduce a prescriptive, evaluative, and obligatory dimension into social life. There are two components to a normative institution: values and norms. Values are conceptions of a preference together with the construction of standards that provide the basis for evaluation (Scott, 2008a). Norms specify how things should be done. Norms provide, sometimes conveniently, the legitimate means to pursue particularly valued ends. Normative institutions define goals and objectives and designate the appropriate ways to pursue those (2008a).

Some values and norms are applicable to all members of a group, whereas others apply only to selected types of actors or positions. Roles may be developed through formal or informal rule creation. Formally, rules are created that apply only to particular members of a group; these rules define the roles of each individual. Roles are defined to carry specified rights and responsibilities and to have varying access to material resources. Informally, roles emerge over time through interactions amongst group members, which differentiate expectations and develop a guide to behavior within the group (Scott \& Davis, 2007). Formal and informal role definitions are common within the interdisciplinary team, where alternative leadership and membership are encouraged.

Normative elements are typically seen as acting as constraints on behaviors, but they also allow for social action. They confer rights and responsibilities; privileges and 
duties; licenses and mandates (Scott, 2008a). The underlying concept remains the focus on social obligation for performance. Conformity or violation of norms involves a large measure of self-evaluation, including heightened remorse and effect on self-respect. Emotions like these provide powerful enticements for group members to comply with prevailing norms. For all the pressures put on an individual, normative institutions stabilize the influence of social beliefs and norms that are internalized and imposed by others.

Policy-regulative elements. As mentioned with the previous elements, institutions constrain and systematize behavior. The regulative element speaks to the rule-setting, monitoring, and sanctioning activities engaged in by the respective institution. The processes involved include the capacity and authority to establish rules, inspect and judge others' conformity to those rules, and when necessary manipulate sanctions (rewards or punishments) in order to influence future behavior (Scott, 2008a). Both formal structures and informal structures engage in sanctioning behaviors. Formal structures include courts, licensing, and accreditation; informal structures include shaming or shunning activities by a social group. North (1990) tied the regulatory concept to team sports; the analogy extends to interdisciplinary teams. He explained that teams consist of written rules and unwritten rules that dictate appropriate conduct and supplement the formal rules. Teams also have contending interests, which require mediation by rules and referees to ensure order. Even though the team is pursuing one set of goals, the individuals share the interest and are on some level pursuing their collective self-interest (DiMaggio \& Powell, 1983). 
It is common to think of oppression and a constraint when one considers regulatory institutions; however, the same regulations can confer power and benefits to actors. The central components of the policy-regulatory element are force, sanctions, and suitability. All three components are mitigated by the presence of formal and informal rules and laws (Scott, 2008a). Unfortunately, authority can be used to legitimize coercive power frameworks, which perpetuate that use of power. To protect against coercive power grabs, a third party acquires the authority to enforce the rules, usually through a contract. The third parties in question must be neutral or the outcomes will be influenced by their behavior and may not serve the intended end (North, 1990). The policyregulative element of institutions renews interest in the role of the government to be rule maker, referee, and enforcer (Skocpol, Evans \& Rueschemeyer, 1985). Many rules and laws are ambiguous enough that, contrary to common belief, there may not be a clear code for conduct. Thus, even rules and laws must be interpreted by regulatory bodies, and differences in interpretation must be resolved.

In summary, organizational institutionalism as a theory to examine the support and legitimacy of IDTs, is a unique approach which, to date, has not been applied to teams in the health care sector. This examination of institutions and teams delves deeper into how many of the structures and behaviors are taken for granted, and thus are infrequently examined. In the same way, each discipline comes with its own set of norms, values, and assumptions conferred through training and work experiences. The influence of professions and disciplines as institutions must be understood when examining teams and their institutional context. 


\section{Professions and Institutions}

The dream did not take power into account. - Paul Starr, 1982

Starr (1982) recounts that "the dream" for medical services delivery was that reason, through the application of both arts and sciences would liberate humankind from suffering at the hands of disease and degradation as thus lead medical science down a virtuous path. Medical science took a different path and it leaves our current system with professional boundaries that are difficult to span, due to issues of authority, dependence, legitimacy, and occupational control. At the core of interdisciplinary teams is the idea of bringing together distinct disciplines to define and solve problems. This is often a challenge due to historical relationships of the professions to institutions and to one another. This section will describe theories of professions and their role as institutions.

Theories of the professions. Friedson (1988), an early sociological theorist of medical professions, suggests that professional attitudes and behaviors are a product of the social constructs and are created by the occupation's ideology, politics, and knowledge (Friedson, 1988). The central distinction he makes between a profession and an occupation is the right to control one's own work. The core characteristics of a profession, as defined by Friedson, are a member has extended training in abstract knowledge and a service orientation (1988). Central to any profession's power is its autonomy. Political and legal privileges, ability to control the production of knowledge, and how that knowledge is used in practice provides a high level of control and power in the workplace. In order to assure conformance to practice guidelines, self-regulation is 
achieved through a ratified code of ethics. Friedson suggested that if the structures that govern medical professional relationships change, accordingly so will the behavior of the individual professions (1988). Power may manifest for various professions through accountability, but even that may come at the expense of practitioner autonomy (Conrad, 2005; Light 1991). Professional rank may change overtime, however professionalization remains essential to the formation of occupational control that professions are privy to in all institutional constructs (Friedson, 1986; Conrad, 2005; Macdonald, 1995). Professions theory proposes that professional control is an alternative to other forms of control, and its focus has been on how to acquire and maintain professional control (Abbott, 1988; Friedson, 1986). These are all important considerations for IDTs as the collaborative nature of IDT work disrupts traditional assumptions and expectations of many health professions.

Professions as institutions. Interdisciplinary teams have professional members, but are not limited to professions; however, the presence of these professions can dictate many behaviors and assumptions within the team. Researchers have recently focused on the institutional environment which lead to more organizational and institutional levels of analysis in examination of professions as institutional constructs. This provides a scope that allowed for the investigation of the role of the state and regulatory powers in establishing professional hierarchies. Kitchener \& Exworthy (2008) proposed that professions are declining in their professional dominance in the health care field is that emerging managerial methods and professions are supplanting managerial more that were more bureaucratic roles. 
The development of professional roles in health care have been the focus of studies about the emerging institutional norms, culture, and regulative elements and the organizational fields that effect professional practice (Leicht, Walter, Sainsaulieu, \& Davies, 2009). An examination of professions must be included when conducting an institutional analysis (Greenwood, 2008), it enhance understanding about how professionals can influence and change their context, both meaningfully and in their own self-interest. Institutions have several mechanisms with which to regulate organizational behavior through various schemas that are developed as a response to values and beliefs rather than market pressures. Professions can enforce of institutional behaviors through policy-regulative, normative, and cultural-cognitive elements (Greenwood, 2008; Scott, 2008b). Institutions are ever changing and professions are part of this change as facilitators in developing emerging organizational fields, through responses to internal and external pressures. The ability of institutions, organizations, and professions to change in order to meet the needs of a changing patient population is significant to assure that the new models can be supported and survive over time. The importance of teams has a resurgence about every ten years, indicating a struggle to achieve full legitimacy within a professionally dominated field.

\section{Interdisciplinary Teams in Health Care}

Drinka and Clark (2000) define IDTs as a group of individuals with diverse training and interests, working together as a unit toward a collaboratively identified goal. Team members consistently collaborate to solve patient issues that are too complex to be addressed by just one discipline. IDTs have formal and informal rules and structures that 
encourage collaborative problem solving by all members of the team. Collaboration is engaged through definition of the team's mission and goals, interdependence of work to treat the patient, and learn to maximize the advantages of disciplinary differences to the benefit of the patient. Leadership is shared in IDTs, and consistently changes with changes in patient needs and team composition. The term "interdisciplinary" is preferred over "interprofessional" in this dissertation due to its inclusive nature, as informal caregivers, family, and the patient may be members of the team.

Early calls for teamwork in health care. In 1915, Cabot wrote that three forms of teamwork would be the most effective way to heal individuals holistically. He called for teamwork between doctors and social workers; doctors, educators, psychologists and ministers; and doctors and patients. The doctor-social worker team was seen to be necessary when,

... a doctor looks for the root cause of most sickness that he is called upon to help, he finds social conditions...vice, ignorance, sweatshops, and poverty. When the social worker analyzes why a family is in need...he finds poor nutrition, bad air, alcoholism, tuberculosis, and injuries from factories staring him in the face" (p.7).

With respect to a doctor's need to team up with educators, psychologists, and ministers, Cabot acknowledges that the physician is only a single person responsible for getting to the root of a patient's ills and “...behind much physical suffering is the mental torment, doubt, fear, worry or remorse..." (p.8). He goes on to emphasize that without 
paying due diligence to the mental ills, it can be impossible to change a patient's bodily suffering. The final team is the patient-doctor team; he explains the dangers to be had in obfuscating truths from the patient about their health. "He must understand what is being done for him if he is to do his part properly" (p.7). Dorothy Rogers (1932) took Cabot's idea one-step further and advocated the interdependence of professional groups in a hospital setting. She included many more occupations in the team conception: RNs, administrative staff, MDs, RDs, researchers, $\mathrm{x}$-ray, and lab technicians all would make decisions in the best interest of the patient. Although these ideas were published between 80 and almost 100 years ago, they did not carry enough weight to change practice at the time.

In 1949, Martin Cherkasky began to realize the ideas of Cabot, Rogers, and others about health teams for use in practice at the Montefiore Hospital Home Health Care Program. He advocated for a focus on the patient in defining treatment regimens, and the inclusion of family and caregiver support in the care for patients, particularly the elderly (Cherkasky, 1949). The Montefiore program expanded the circle of care beyond the medical clinic, and included the home and community as part of the treatment setting. The care coordination of home-based services is considered by many to be the origin of health care teams; however, the Montefiore case was more focused on the coordination of services rather than collaborative teamwork across disciplines. The traditional hierarchy of power was adhered to within the Montefiore teams. 
George Silver, a colleague of Cherkasky's, advocated for a collaborative model of care. In 1958, he extended the health care team beyond service coordination to operationalizing teamwork as a process such that responsibility is divided, there are delineated individual roles, and team members "accept each other's professional competence" (1958, p.33). Silver's team model consisted of a physician, a psychiatric social worker, and a public health nurse. Team members would meet with families, either at home or at the office. Later the team would meet to discuss the findings and a treatment or action plan would be developed. The plan was discussed with the families and responsibilities were distributed amongst team members. New members would be added as the need arose, but the team would schedule regular conference to ensure the exchange of new information and developments. Team interaction was essential, according to Silver: "Every contact is an opportunity to tap the knowledge and skills of all team members because the intercommunication of the team members leads to the exchange of skills and interests" (1958, p.34). This is the foundation for how IDTs are actualized in collaborative practice today.

In 1982, Theodore Brown proposed that teams were more of an ad-hoc condition than a systematic and empirically demonstrated approach to care. Brown raised questions about motivations for teamwork, the quality of relationships among team members, and the levels at which teams function. In his concluding comments, Brown directs researchers to study health care teams to provide greater empirical evidence of team characteristics and effectiveness. Twelve years later Madeline Schmitt (1994) reviewed the major events in interdisciplinary health care delivery. In her review, she 
included education and research efforts that supported the further development of the interdisciplinary literature. Schmitt emphasized the relevance of IDT care for particular settings and patient populations. Acute care, community mental health centers, chronic illness, geriatric care, and primary care were all identified as being appropriate fits for an IDT approach. Schmitt also outlined how interprofessional education initiatives led to a greater acceptance of IDT in practice.

In 1996, David Patrick Ryan examined the history of teams in the delivery of mental health services and suggested that there were implications for more traditional team-based care in gerontology. Community mental health movements echoed community health care in their interest to adapt and explore new models of care delivery. These new models confronted the issues typically experienced among health care professionals, while creating an opportunity for non-traditional providers to take part in team care. Ryan concluded that the changes occurring in mental health would amount to a paradigm shift in health care and that education and training programs would need to develop the appropriate knowledge and skills to practice in a quickly changing environment (1996).

DeWitt Baldwin (2007) took a more historical view and examined IDT activities from World War II through 1996. He explained that IDTs are an accepted means to care for the poor and underserved populations by providing them comprehensive and continuous care. These cases traditionally utilize medical and social services concurrently. The connection between providing health care for the poor and 
underserved is important in considering the impacts of team care on the social safety net and establishing the role of the federal government in promoting team care models. Private foundations have also played a substantive role in encouraging and supporting IDT education and practice. Baldwin highlighted the role of the improvement movement in grounding team-based models of care around the concepts of quality, efficiency, and effectiveness. Baldwin concludes that increased attention needs to be paid to the collaborative work relationships, rather than merely advocating for a team structure.

\section{Institutional support for interdisciplinary teams. Current research on IDTs} has underutilized theory to describe and help understand teams and their work settings (Reeves 2010). Terms like power, leadership, and culture are often associated with team studies; however, few studies use the theoretical backgrounds of these topics to provide greater insights. Two principle elements of institutions at a micro level provide insight into interdisciplinary teamwork: relational factors and procedural factors. Each element represents a level of analysis and understanding of how researchers create meaning for teams in health care. Rational and procedural elements are internally constructed institutions that originate within the team; the external elements originate from environmentally constructed institutions are discussed in the previous section.

Relational elements. The relational elements of teams are represented in the literature through discussions focused on hierarchy, professional power, socialization, team composition, team roles, and team processes. The division of labor within many health institutions is hierarchical, creating a vertical authority, where senior members 
supervise the junior members. In most interdisciplinary teams, physicians are given a position atop the hierarchy; this professional dominance is historically documented (Friedson, 1988; Reeves, 2010; Starr, 1982). It is well documented that individuals with more experience are positioned higher in the hierarchy, leading to hierarchies within professions as well as between professions (Abbott, 1988; Friedson, 1986). These structures of hierarchy can be a barrier to interdisciplinary collaboration in teams. Intimidation by superiors of junior members of teams may stifle valuable contributions, or necessary corrections of mistakes made by senior members (DiPalma, 2004; Mahmood-Yousuf, Munday, King \& Dale, 2008; Sexton, Thomas \& Helmreich, 2000).

Professional power is a complex issue within interdisciplinary teams. Medicine was the first health care occupation to professionalize itself and leverage its knowledge and skills to create great influence in all health matters. The centrality of the medical profession to all health care matters was rarely questioned and lead to a great level of social status and financial reward for members of the medical profession (Friedson, 1988; Starr, 1982). The nature of medicine's power is not just limited to decisions about a patient's diagnosis and treatment, but also includes influence in policymaking around regulation and rule making for health organizations (Fiorelli, 1998; Freidman, 1962; Gardezi et al., 2009; Hugman, 1991). The adaptation and usage of interdisciplinary teams can hinge on medicine's willingness to share power (Mackay, 2004); even if there is a willingness to share power at the surface, the extent to which that power sharing is evident in practice can be questionable (Gibbon, 1999). Besides identifying who has power, there is also the other side of the power relationship to consider as well as who 
has power, how do those without power respond? Resistance to power has been observed as an equally detrimental force (Foucault 1978). Power resistance may be passive, such as apathy toward teamwork and not "pulling one's weight" or active, as seen in sabotage efforts of other team members or team projects (Delva, Jamieson \& Lemieux, 2008; Reeves, 2010). IDT relationships require daily management to assure integrity and legitimacy of the team and that they are working at the most productive levels.

Team composition reflects the number and membership of the team and is a key determinant of team functions. The mix of professions within a team can contradict each other, as they will be the product of different professional socialization processes. Socialization is the process by which team members will acquire norms, values, and attitudes associated within a particular discipline. Socialization creates a loyalty of the individual to their discipline over the team (Becker, 1961; Blane, 2003). The greater the number of disciplines involved in a team, the greater potential there is for distrust and professional fragmentation (Heinemann \& Zeis, 2002; Reeves, 2010; Wilson-Barnett, 1989). The next issue with team composition is the number of team members. There are diminishing returns, as the team size gets too large. Greater diversity and number provides more expertise and perspective, but too many members makes scheduling meetings, coordinating tasks, and conducting collaborative problem-solving highly problematic (Douglas, 2000; Handy, 1999; Reeves et al., 2006; Williams \& Laungani, 1999). Another problem with large teams is the potential for sub-teams to form and dissociate further from the initial team. 
The final relational characteristic of IDTs is team roles; the formation and preservation of clear roles is essential for optimal team functioning (West \& Markiowicz, 2004). Clear roles define the nature of each team member's tasks, responsibilities, and scope of practice. Since many disciplines are monitored by a third party through licensing and scope of practice laws at the state level, role delineation is important to avoid professional boundary infringement (Booth \& Hewison, 2002; Reeves, 2010; Stark, Stronach \& Warne, 2002). One role is that of team leader (Martin \& Rogers, 2004). A team leader is the individual who influences others through their ability to motivate, make decisions, and encourage innovation (Cook, 2003). The range of leadership approaches goes from transactional to transformational (Bass, 1999). Transactional leaders are authoritative, work in isolation from the team, and make decisions without including team members, which is common in multidisciplinary teams. Transformational leaders work more democratically and work with members to promote collaborative problem solving.

Procedural elements. The procedural factors of teams are represented in the literature through discussions around routines, task delegation, and time and space. Routines can be seen as a course of standardized actions or procedures that are followed regularly across health care settings; they provide a cognitive map for individuals and institutions to undertake all the tasks that must be accomplished in a stable and predictable way. However, routines can also over systematize the functions of the teams, and little innovation or unique attention will be paid to families and patients (Healey, Undre \& Vincent, 2006). Weekly meetings intended to facilitate collaborative problem 
solving actually included very little collaboration and, in fact, Lewin and Reeves (2011) found that many professionals did not attend these meetings and few decisions or changes were made. They further concluded that the meeting served the sole purpose of providing a visible demonstration of teamwork that can be monitored and tracked, but the quality of it remained unevaluated.

One of the central effects discussed in the previous section is the professionalization within health care professions; this specialized level of expertise is protected from infringement from other professional groups (Friedson, 1986). Task delegation is increasingly gaining ground as one way to share knowledge and responsibility, and provide individuals with work opportunities they would not have had otherwise. Task delegation can be a legislated permission for licensed professionals to hand over specific pieces of their workload, and train another qualified member of the team to do those tasks (1986). Delegation allows for greater flexibility in how care is delivered in the face of increasing demand; however, professional boundaries are still a sensitive area and doctors and nurses are not always willing to share pieces of their scopes of practice (Friedson, 1986; Reeves, 2010). Delegated tasks are typically on the 'low-status' or 'dirty-work' end of the spectrum and may threaten the professional status of one profession in relation to another, refining a hierarchy, instead of leveling it (David, 2010; Ward, 1991).

The final procedural characteristic important when examining teams is time and space. According to Durkheim (1976), time and space are intertwined with the social 
organization of work. Spatial boundaries fragment many health services needed by a patient; these boundaries also separate providers who should be co-located (Armstrong, 1985; Grinspun, 2007; Pryor, 2008). Time is perceived as being necessary for teams to develop trust, respect, and mutual understandings about roles and competencies (Jaques, 2002). Simply co-locating teams is not enough to develop a team. To reap the benefits of a team-based model of delivery management a team must engage in collaborative training and team-building exercises, with downtime for team members to understand each other (Mitchell, Parker, Giles \& White, 2010; Proudfoot et al., 2009). However, time, even if socially defined, is always in short supply and matching up what is known about team development and the realities of practice can be very challenging (Allen, 2002; Engestrom, 1999).

\section{Interdisciplinary teams and the Program for All-Inclusive Care of the}

Elderly. In the 1970s, team initiatives began to change their primary and family care emphasis to address the issues of particular populations. Rehabilitation, dialysis, and hospice began to appear in the health team literature (Tsukuda, 2005). Older adults in particular received more attention as a priority area for team-based care initiatives. Williams stated that teamwork needs to be a core value in geriatric services: "It is clearly impossible for anyone professional to address adequately the full range of problems older patients often present, usually at times of crisis -- medical or social....to be a 'complete geriatrician' means to be a team geriatrician" (1986, p. 349). The perpetual struggle of health care to provide effective and affordable care is of acute interest when anticipating the effect of aging baby-boomers on current health care resources. Currently, the 
fragmented nature of health service delivery and the financing mechanisms to pay for care are not meeting the needs of patients (IOM, 2008; Stone, 2000). The shift in emphasis, in long-term care to home and community-based care is anticipated to have many benefits including being a more affordable option that provides elders with better coordination of services, using a more collaborative decision-making process that will improve patient outcomes, and support connectivity between housing, social services and medical care.

Program history and performance. The first PACE program was established in 1973, in San Francisco (Bloom, 2011). On Lok was founded under the leadership of Marie-Louise Ansak and a $\$ 2000$ federal grant. The values embedded at the core of the PACE program include accountable care, health homes, patient-centeredness, and an IDT delivery model (Bloom, 2011). By 1979, the On Lok program was receiving Medicaid reimbursement for adult day services and receiving funds from the Department of Health and Human Services to develop a model that could be replicated in other states (Hirth, Baksins, \& Dever-Bumba, 2009). PACE was one of two demonstration programs to combine acute and long-term care for the frail elderly at the national level. The first, started in 1985, was the Social Health Maintenance Organization (SHMO) (Newcomer, Harrington \& Friedlob, 1990). Funded by Medicare, there are currently four programs still operating in the U.S. as demonstrations 28 years later. In contrast, PACE programs have proved to be a viable model for the frail elderly and have progressed beyond the demonstration phase (Gross, Temkin-Greener, Kunitz, \& Mukamel, 2004). 
In 1981, On Lok received waivers from Medicare and Medicaid to test a new financing system that would allow a single capitation rate to be paid per participant per month (Eng, Pedulla-Eleazer, McCann, \& Fox, 1997). On Lok assumed full risk for cost coverage and still managed to grow and sustain its services. PACE programs are a threeway partnership among the provider, the state Medicaid agency, and CMS (NPA, 2004).

Figure 2.1 illustrates the growth and development of PACE programs.

Figure 2.1: PACE Program Growth 1997-2013

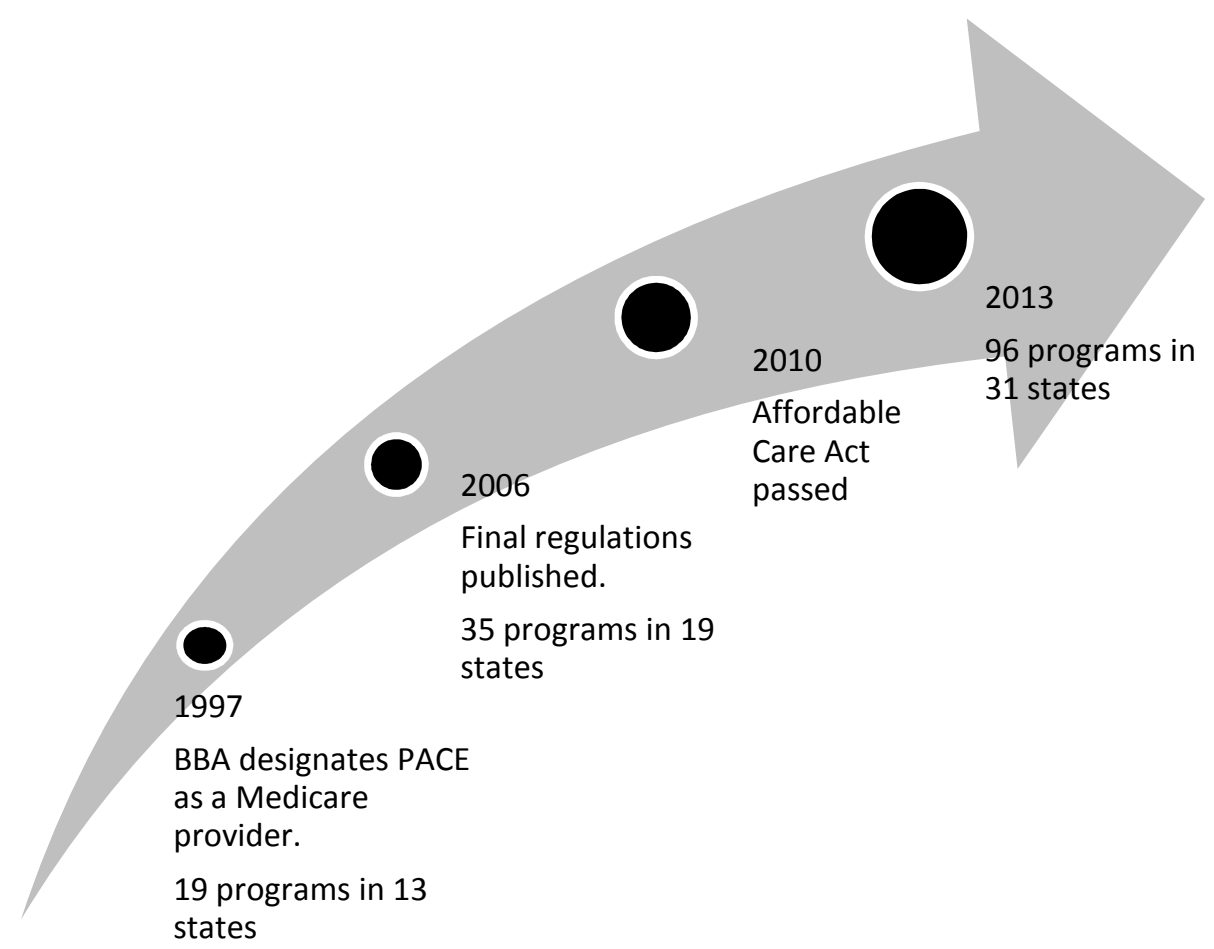

The 2006 final regulations made a terminology change from referring to the team as multidisciplinary to interdisciplinary. This was significant in conveying the commitment to a collaborative care model. Along with the requirements of a minimum of eleven different disciplines participating in the IDT, the PACE regulations also state 
that those members should have at least one year experience in working with the elderly (Programs for All Inclusive Care of the Elderly, 2006). The IDT is responsible for the initial and periodic assessments, the plan of care and coordinating 24-hour care delivery. The primary care provider (PCP) serves as the gatekeeper to the participants' use of medical specialists and in-patient care, but they must collaborate with the rest of the IDT and keep them informed of the medical conditions of the participants. Team leadership is at the discretion of the parent organization or the IDT, and this role varies greatly amongst programs and teams.

The average participant in PACE is 80 years old, has an average of eight medical conditions, and three limitations of activities of daily living (ADLs) (Temkin-Greener, 2006). Approximately half of PACE enrollees suffer some form of dementia or Alzheimer's disease (Gross, Temkin-Greener, Kunitz \& Mukamel, 2004; NPA, 2010). Participants and their families work closely with their IDT to develop individualized care plans that are based on their personal values, goals, and objectives. Before participants can be enrolled they are assessed medically and socially to determine needs and qualification (Gross, Temkin-Greener, Kunitz \& Mukamel, 2004). Also prior to enrollment, the participants must give up their personal health care provider and agree to receive all their care through the PACE IDT. PACE care is provided by a federally mandated IDT and many parent organizations contract with other providers to fill any needs of the patient (Bloom, 2011). The team includes eleven different disciplines: physician, registered nurse, social worker, recreational therapist, physical therapist, occupational therapist, registered dietician, home care coordinator, personal care 
assistant, drivers, and a center manager or administrator (Mukamel et al., 2006). The team is federally required to meet regularly (most meet every day) to discuss participants and give updates on health and well-being status.

PACE enrollees have lower rates of nursing home admissions, shorter hospital stays, lower mortality rates and better self-reported health and quality of life as compared to non-PACE populations (Chatterji et. al., 1998; Petigara \& Anderson, 2009). Costs for PACE enrollees are 16-38 percent lower than Medicare fee-for-service costs for a frail elderly population, and 5-15 percent lower than costs for comparable Medicaid beneficiaries (Bodenheimer, 1999; Petigara \& Anderson, 2009; White, 1998). PACE is associated with many statistically significant outcomes including lower nursing home visits, fewer hospitalizations, reduced nursing home admissions, greater survival rates, better health and quality of life and satisfaction with care (Grabowski, 2006). Many of the projected benefits of the PACE model have come to be a reality; however, there are still barriers to growth and expansion of PACE and similar team-based programs. Researchers theorize that these outcomes may have more to do with institutional norms and resistance to change than with the PACE program itself (Heinemann and Zeiss, 2002; Lynch, Hernandez, and Estes, 2008; Tsukuda, 2005).

The concept of a medical home is gaining legitimacy as an important part of health care. PACE organizations have functioned as a medical home since their inception, they provide care 24 hours a day, seven days a week over time and across all care settings including preventive, primary, acute, and LTC (Bloom, 2011). One of the 
core values of PACE is maintaining the independence of the individual for as long as possible. PACE programs emphasize the impact on health and well-being of keeping an individual in their home or community over institutional care.

Current PACE regulation and policy. PACE regulation and policy oversees who qualifies as an enrollee, the capitated payment system, and requirements for health workers. Under current CMS regulations PACE participants are at least 55 years old and require nursing home level care. Participants receive all Medicare and Medicaid covered services as well as social support from their PACE organizations (Bloom, 2011). Access to a PACE program is also limited by geographic "catchment areas", which may be defined by zip code, county boundaries, or other criteria developed by the state (Gross, Temkin-Greener, Kunitz \& Mukamel, 2004). Each state is subject to its own range of issues, which affects the way they support, develop, and maintain their long-term care systems (NPA, 2004). This state level variation and support can create obstacles to maintaining the PACE model of care and the high levels of quality expected to maintain PACE provider designation (NPA, 2004).

As discussed above, the PACE payment model is one of the distinctive characteristics of the PACE program. The one policy mechanism that has been repeatedly proposed in order to control the cost of publicly funded long-term care is the use of capitation payments (Grabowski, 2006; Rudolph \& Lubitz, 1999). The joint Medicare-Medicaid capitated payment calculates a flat rate per participant per month. The funds allow for the provision of services outside the Medicare and Medicaid 
reimbursement schedules. Capitated payment also allows for salary payment of health professionals instead of the traditional fee-for-service payment model. Regardless of services provided and providers utilized, the POs are fully accountable for the quality and cost of all services, even if the cost exceeds the capitated rate (Bloom, 2011; Eng, Pedulla, Eleazer, McCann \& Fox, 1997).

Capitated programs such as the PACE program offer the opportunity for payment to be more equitably distributed amongst team members. Non-traditional payment systems will be necessary in order to support widespread use of work teams in long-term care. Although Medicare does not itself pay a care coordination fee, many of the LTC programs will pay their providers a salary and this may be the place to integrate payment for time spent in team meetings. The more formalized the processes of work teams become, the more likely reimbursement can be sought. PACE substantiates the assumption of the positive nature and contribution of work teams and the cost and care benefits of them as well (Cooper \& Fishman, 2003). System level funding allows for discretion in leadership and role definition within those teams. Paraprofessionals, who are often held at the bottom of the hierarchy, with experience may become the most valuable and knowledgeable member of a team. It has yet to be demonstrated if a new or expanded role can make its way into standard care. However, there is certainly potential to provide more power and influence over care decisions to the staff members who know the patient best. This shift in leadership and care delivery is one of many focus areas of the Affordable Care Act. 
POs embody many of the systematic changes called for by health reform efforts. The Affordable Care Act (ACA) presents several opportunities that may encourage greater PACE growth. Within CMS, the ACA established an office that focuses solely on the dually eligible (Bloom, 2011). The office hopes to improve integration of care, improve quality measurement, and increase access to home and community based longterm care (Kaiser Family Foundation, 2011). The Centers for Medicare and Medicaid Services (CMS) also developed the Center for Medicare and Medicaid Innovation, to test unique models of care provision in order to improve care quality while reducing the overall cost. Both of these developments create an opportunity to raise the awareness of the PACE model, and may begin to address barriers to growth. The ACA has also created financial incentives for states to increase their use of community-based long-term care instead of relying on the institutional model of care including money follows the person, Medicaid health homes, and LTC system rebalancing (Bloom, 2011).

Barriers to growth of PACE. Despite the success of many POs across the country, growth in numbers of PACE programs has not matched expectations. The BBA authorized 40 new PACE programs for 1997 and 20 new programs every year after, allowing for a potential 340 PACE programs to be operating in the United States by 2013. However, currently there are 91 programs, operating in 31states (NPA, 2013). Some barriers are the time and monetary investment, support of state Medicaid agencies, and recruitment of adequate numbers and types of provider to fulfill the IDT requirement (Bloom, 2011). Lynch, Hernandez, and Estes (2008) identified eight barriers to PACE program growth and dissemination. The first barrier is that PACE is unappealing to older 
adults. Potential participants are also put off by the requirement that they use a PACE physician. At this time in life a physician may have a long-standing relationship with the participant and to give up that relationship is asking too much (Lynch, 2008; Petigara \& Anderson, 2009). Required participation at an adult day center as required in PACE provides an opportunity for informal interactions with their IDT. Individuals have stated that they would rather go to the day center when they wanted, and receive most of the services in their home (Gross, Temkin-Greener, Kuntiz \& Mukamel, 2004; Lynch, Hernandez \&Estes, 2008).

Second is that non-profit providers have inadequate resources to start up a PACE program. Total startup expenses typically cost between 1-2 million dollars, depending on a provider's existing building and equipment assets (NPA, 2004). Many states were able to take advantage of the demonstration grants available from large national foundations; however, since PACE is no longer a demonstration, those grants are no longer available (Petigara \& Anderson, 2009). The third barrier is labor shortages, as existing PACE sites often cannot grow due to the lack of available and qualified providers. Each PACE team can only care for up to $120-150$ participants, after which a new team must be assembled. Providers are required to have one-year experience in treating the elderly. PACE organizations can have trouble offering competitive benefits packages and wages (Lynch, Hernandez \&Estes, 2008; Gross, Temkin-Greener, Kuntiz \& Mukamel, 2004).The fourth barrier is the lack of for-profit providers entering the PACE market. Even though the 1997 BBA authorized development of 10 for-profit PACE sites, not one was developed. Reasons included the unpredictable nature of capitation rates and uncertainties of 
assumption of financial risk in caring for the frail elderly; these have discouraged any forprofit providers from coming forward (Gross, Temkin-Greener, Kuntiz \& Mukamel, 2004). Fifth is the lack of marketing and outreach for PACE programs nationwide. Older adults and their families are unaware of the PACE model. The non-profit status of the POs leaves little resources to develop marketing and outreach (Lynch, Hernandez \&Estes, 2008). PACE programs also have a visibility issues amongst primary care providers in general. The sixth barrier is a lack of state support, through limiting enrollments from the Medicaid programs. The "wood-work effect" was anticipated by state officials, citing that individual would "come out of the wood-work" in order to take advantage of PACE services who would not done so otherwise. The fear was that this would inflate state costs, overall. There is also competition between state provided home and community-based services and PACE programs instead of cooperation (Gross, 2004). Seventh is that PACE is unaffordable to middle-income individuals. There are barriers to non-Medicaid enrollees in gaining access to PACE programs. PACE regulations were developed before many programs were available and may have codified practices that limit necessary flexibility and growth. Finally, there are ongoing struggles to realizing the PACE model in rural areas, where all of the issues described above are still in effect, with even fewer resources locally available to maintain a comprehensive program like PACE (Lynch, 2008).

\section{Conclusion}

There are a number of foundational theoretical works upon which this study was developed; there is a strong basis for examining the institutional dynamics of team-based 
practice in the care of older adults. Examinations of institutional restructuring are well established with organizational institutionalism, but few look at engendering and legitimization of the care delivery model supporting an IDT. Studies tend to examine one profession at a time; not how professions are brought together for a common purpose. Scott's framework for analyzing institutions will provide a broad-view of the policyregulative, normative, and cultural-cognitive elements that create the context in which institutions work. Reeves' framework provides procedural and relational elements, which are internally constructed institutions within and in close proximity to the team.

Another unique feature in using Scott and Reeves as a framework is that it provides a guide to examining external, formalized elements of the institution, and investigating the internal and less formalized elements created by integrating distinct professions into a singular care-delivery unit. The history of teams in health care has evolved over an extensive period of time. Institutions are notoriously slow to implement changes. Revisiting the circumstances that created the current context provides a basis to understand health care organizations. The literature cited previously gave the historical background of teams in health care and how teams came to be the goal for geriatric care. The development of IDTs continues to provide an opportune moment to examine the current institutional context, in supporting teams as a care delivery model for older adults. The PACE model is an example of a sustained model of participant-centered, team-based, collaborative care, and it provides an example of how to support teams for the long-term and highlights what improvements are still needed to be made in the changing health care environment. 


\section{Chapter 3 Methodology}

\section{Introduction}

This chapter will give an overview of the qualitative multi-case study research design. The goal of this study is to explore how institutional structures affect the support and legitimization of interdisciplinary team (IDT) care for older adults. This case study used a qualitative methodology to illuminate the institutional context for IDT support. The Programs for All-Inclusive Care for the Elderly (PACE) were selected as the cohort for this study because every PACE program is required to support an interdisciplinary team made up of 11 different disciplines. Based on the literature reviewed in Chapter Two, five contextual elements were identified that affect levels of support given to IDTs: (1) cultural-cognitive elements, (2) normative elements, (3) policy-regulative elements, (4) relational elements, and (5) procedural elements. Due to the complex nature of examining institutional context, a case study method was selected as the best way to explore the context variation among these elements across selected sites.

\section{Research Theory}

There is a deficiency in the published literature in defining the health care institutional concepts that contribute to the use of IDTs in the care of older adults. It is appropriate, then, to begin with an examination of the organizations that have committed to using IDTs in the care of older adults, and study how these institutions legitimate and support team-based practice. Therefore, the focus of this research is to examine the institutional context of organizations' adaptation and continued support for the PACE 
IDT model of care across five different elements of institutional context using a case study design.

Case study, as a research method, is an effective research strategy for answering questions using data from multiple sources and informants in reference to a particular situation (Yin, 2009). The case study method is most useful when the research focuses on a unique and bounded system. The characteristics of the system are what make the situation unique and are the focus of the study (Stake, 2006). The case study follows a structured framework and is useful in investigating a complex contemporary phenomenon using multiple data sources (Yin, 2009).

The qualitative approach for this case study identified seven different PACE programs in six states. Some aspects of the PACE program are legislated in order to institutionalize practice; however, apart from those core elements there could be significant variation in implementation of the program and the IDT environment across organizations. Thirty-two qualitative interviews provided the data to construct the aggregated case. The focus of the case study was the institutions that govern the PACE organization and its IDT.

\section{Research Question}

The research question is:

What elements of institutional context support the use of interdisciplinary teams in the care of older adults? 
This research traced each of the PACE programs within each of the selected organizations from the macro institutional elements to the micro institutional elements illuminating how support is actualized for teams or, if support is not actualized, how barriers are created. Table 3.1 defines the core concepts of the research question.

Table 3.1: Definitions for Core Concepts of Research Question

\begin{tabular}{lll}
\hline Concept & Definition & Source \\
\hline Institution & $\begin{array}{l}\text { The cognitive, normative, and regulative structures } \\
\text { and activities that provide stability and meaning to } \\
\text { social behavior }\end{array}$ & Scott, 2008 \\
\hline $\begin{array}{l}\text { Institutional } \\
\text { Context }\end{array}$ & $\begin{array}{l}\text { The combination of internal and external } \\
\text { environments that define roles, responsibilities, } \\
\text { resources toward goal achievement }\end{array}$ & $\begin{array}{l}\text { Ducanis and } \\
\text { Golin, 1976 }\end{array}$ \\
\hline $\begin{array}{l}\text { Interdisciplinary } \\
\text { Team }\end{array}$ & $\begin{array}{l}\text { A group of individuals with diverse training and } \\
\text { interests, working together as a unit to identify goals } \\
\text { and work towards them collaboratively }\end{array}$ & $\begin{array}{l}\text { Drinka and Clark, } \\
\text { 2000 }\end{array}$ \\
\hline Legitimacy & $\begin{array}{l}\text { A generalized perception or assumption that the } \\
\text { actions of an entity are appropriate within some } \\
\text { socially constructed system of norms, values, and } \\
\text { beliefs }\end{array}$ & Suchman, 1995 \\
& & \\
\hline
\end{tabular}

As discussed in Chapter Two, a high-functioning team finds support and legitimization through its institutional context. A high functioning team has the following characteristics (Yeatts \& Hyten, 1998):

- Clear goals and a shared sense of purpose and commitment to achieving them

- Clearly understood roles and responsibilities

- Clear and regular communication

- Mutual trust, respect, understanding and support

- Recognition and appreciation of contributions of all team members

- Effective leadership

- Mechanisms and strategies for team tasks

- Appropriate organizational structures, including regular meetings 
Based on the literature reviewed, it is proposed that the contextual elements of cultural-cognitive, normative, policy-regulative, relational and procedural would be the foundation of support of the IDT model of care. Table 3.2 delineates the operational definitions for the five contextual elements of interest.

Table 3.2: Operational definitions of context elements

\begin{tabular}{ll}
\hline $\begin{array}{l}\text { Contextual } \\
\text { Element }\end{array}$ & Operational Definition \\
\hline $\begin{array}{l}\text { Cultural- } \\
\text { Cognitive }\end{array}$ & $\begin{array}{l}\text { An institution's shared conceptions that constitute the nature of social reality and } \\
\text { the frames that create meaning (Scott, 2008). Cultural-Cognitive elements take } \\
\text { into account objective conditions and the key informant's subjective } \\
\text { interpretation of them. }\end{array}$ \\
\hline Normative & $\begin{array}{l}\text { Rules introduce a prescriptive, evaluative, and obligatory dimension to social } \\
\text { life. The normative element constructs the values and norms that define goals } \\
\text { and objectives and the appropriate way to pursue them (Scott, 2008). Values and } \\
\text { norms apply to all members of the IDT, while other norms will apply to only } \\
\text { select team members. This will create roles within the IDT, including particular } \\
\text { roles that define and carry specific responsibilities and scopes of practice. }\end{array}$ \\
\hline $\begin{array}{l}\text { Policy- } \\
\text { Regulative }\end{array}$ & $\begin{array}{l}\text { Rule setting, monitoring, and sanctioning activities that operates through a } \\
\text { diffusion of formal and informal mechanisms in an attempt to influence behavior } \\
\text { (Scott, 2008). }\end{array}$ \\
\hline Relational & $\begin{array}{l}\text { Relationships amongst professions and disciplines within the IDT and how the } \\
\text { IDT interacts with its organizational environment (Reeves, 2010). An essential } \\
\text { aspect of the IDT is the ability of two or more disciplines to work together, with } \\
\text { overlapping roles, status differences, and viewpoints that can lead to conflict and } \\
\text { inefficiencies. Relational elements are a determinant of team effectiveness } \\
\text { (Ducanis \& Golin, 1979; Reeves, 2010). }\end{array}$ \\
\hline $\begin{array}{l}\text { Factors that affect the work of the IDT, by influencing how they are carried out } \\
\text { across different workplaces within the team construction (Reeves, 2010). Each } \\
\text { organization has found the mental processes by which it works to meet its goals } \\
\text { and examination of the way resources are allocated and knowledge shared is } \\
\text { crucial. }\end{array}$ \\
\hline Procedural
\end{tabular}

Conceptual framework. Figure 3.1 illustrates the conceptual framework for this research project. The institutional context is defined by internal and external elements to the PACE IDT. This research will examine the extent to which the institutional context 
influences the ability of the PACE program to facilitate support or create barriers for the IDT. Attention will also be paid to any influence that the PACE program use of an IDT exerts over other existing structures or programs.

Selection criteria. Stake (2006) suggests that cases be selected to maximize what can be learned. It is important to take into consideration the purpose of the study and examine cases that will provide greatest understanding of the context. Stake suggests three main criteria for selecting cases: (1) is the case relevant to the research question; (2) do the cases provide diversity across contexts; (3) do the cases provide good opportunities to learn about complexity and context (2006, p.23). Cases for this study were selected based on selection criteria to reduce the number of probable sites, and then sites were selected to maximize context variation. However, Stake does note that sometimes it is necessary to select cases, which are accessible and open to being part of the study (2006). As defined through the literature and available data on PACE organizations (PO), the case selection in this study occurred in two phases. Phase one was elimination, to reduce the pool of POs from the existing 89 programs. Two criteria were used in phase one: PACE certification and having had at least 5 years of operation as a PACE certified provider. Phase two was variation, where characteristics that would create the greatest understanding of context were delineated for each PO, as identified from the literature review in Chapter Two. Purposive sampling was used to select the cases in this phase for the most context variation. These criteria include: organizational form, overall long-term services and supports performance, percentage of the population 
over the age of 65 , percentage of those over 65 who belong to a minority group, those over 65 years that are living in poverty, and the state position on the

Figure 3.1: Conceptual framework for research

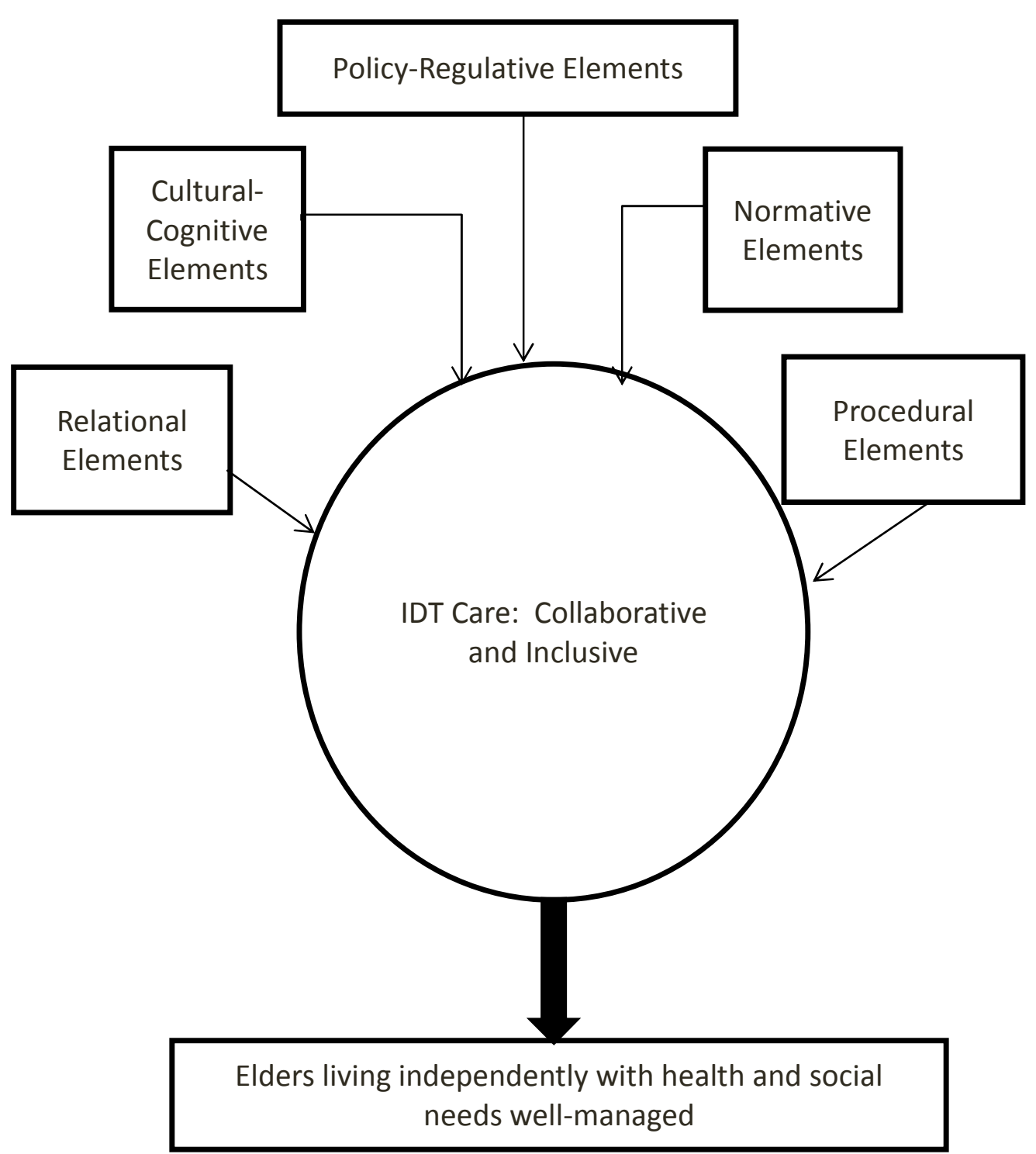


Affordable Care Act (ACA). Each of the selection criteria are described fully in the following section.

Criteria for elimination. Two criteria were identified, that would reduce the number of programs for recruitment efforts: PACE certification and years of operation. These criteria eliminate programs that would not have the relevant experience to participate in the study.

PACE certification. Under sections 1894 \& 1934 of the Social Security Act, a PO must be both a participant in Medicare and Medicaid, have the full support of the State Administrative Agency, and have an approved application from CMS (CMS, 2006). Meeting these requirements indicated that the PO will adhere to two conditions that cannot be waived: a capitated integrated financing model, and assumption of all financial risk. This criterion reflects the normative and policy-regulative elements. The normative element was reflected as recognition as a PO introduces specific rules that were prescribed and used to evaluate the goals and values of the PO. The policy-regulative element was reflected in the regulatory framework provided by the joint Medicare and Medicaid funding of POs. There is further influence of the policy-regulative element in the requirement of obtaining support from the State Administrative Agency in order to gain approval as a PACE provider.

This criterion eliminated three of the 89 PACE programs from consideration, due to their pre-PACE status. 
Five years of operations as a PACE organization. There are structural inertia forces that can inhibit the adaptation of new models of care delivery (Hannan \& Freeman, 1984). Balas, Boren, and Brown (2000) stated that it could take five to seven years to adopt new best practices. Thus, the second elimination requirement was that the organization had at least five years' experience as a PO. This provided adequate time to experience some turnover, communication difficulties, and test problem-solving mechanisms. The time designation also provided that all POs in this study had experienced legislative sessions within their state and two Medicare audits. The elements reflected in these criteria are the cultural-cognitive and normative elements. The PACE organization cultural-cognitive element will change over time and team functioning will evolve the longer team members are working under the PACE IDT model. This criterion eliminated 46 PACE programs from consideration.

Criteria for variation. In order to prioritize recruitment of organizations and assure an adequate level of variation amongst cases, four criteria were examined: organizational form, overall long-term services and supports rating, demographic characteristics, and state position on the Affordable Care Act.

Organizational forms. The National PACE Association defines three categories of PO: health system, long-term care provider, and a freestanding PO (2012). A health system consists of all organizations, people and actions whose primary intent is to promote, restore or maintain health. The health system makes efforts to influence determinants of health as well as direct health promoting activities. A long-term care 
provider is an organization that provides an array of long-term care supports and services, including assisted living, home health care, independent housing, care coordination, hospice, or palliative care; in these sites a PACE program will be one of many services that are offered. A freestanding PO is a nonprofit that is only a PACE provider. All staff and resources are for PACE program purposes only.

The organization that houses the PO will directly affect several aspects of the PO context, specifically in terms of the policy-regulative and procedural elements. The organizational form can dictate all layers of administration, resources available, and governance structure. As discussed in Chapter Two, there are five primary ways that organizational forms can affect team function: (1) provide an external definition of structures and routines, (2) external logics superseding internal logics, (3) level of generalization of beliefs and (4) values to meet a diverse array of health needs, and (5) the level of standardization within systems of control over activities and actors (Ducanis \&Golin, 1979; Lammers \&Barbour, 2006; Scott, 2008a; Scott \& Meyer, 1994). These qualities were observed in organizational rule setting and utilization of waivers, as well as team processes and governing hierarchies.

Overall LTSS system performance. The State Long-Term Services and Supports (LTSS) Scorecard was an effort by the SCAN Foundation, AARP, and The Commonwealth Fund to measure state level performance of LTSS systems within each state that provide services to older adults (Reinhard, Kassner, Houser \& Mollica, 2011). The LTSS performance criterion is a combined measure of four key dimensions of LTSS 
system performance: (1) affordability and access; (2) choice of setting and provider; (3) the quality of life and care; and (4) support of family caregivers (Reinhard, Kassner, Houser \& Mollica, 2011). Affordability and access represent the ease with which consumers can find and afford the LTSS services they need. It also takes into account the capacity of the safety net to provide for those who cannot afford services. Choice of setting and provider signifies the level of commitment to a person-centered approach. As discussed in Chapter Two, a person-centered approach is perceived to be a critical piece of culture change within the long-term care spectrum of services. There is a belief that allowing consumers to exercise choice and control over where they receive services and who provides those services will improve the quality of life and care (Cooper \& Fishman, 2003; Reeves, 2010; Reinhard, Kassner, Houser \& Mollica, 2011). To characterize the quality of life and care, researchers examined (Reinhard, Kassner, Houser \& Mollica, 2011) how services maximize positive outcomes, and the level of respect given to consumers. Support for family caregivers represents how well the caregiver needs are met, so that caregiver stress is kept to a minimum. The final dimension, effective transitions and organization of care, measures how well services are coordinated and integrated with health and social services. These five dimensions were used to establish a ranking of all states, and then divided into quartiles. For these selection criteria, sites were arrayed across a range of quartiles as an indication of a state's pre-established commitment to provide high-quality services for older adults and the poor. These measures touched on each of the context elements. 
Demographic characteristics. Three demographic indicators were used to create greater variation amongst sites. The three demographic points examined for this purpose were the percent of the city population over the age of 65 , percent of minority persons over the age of 65 , and the poverty rate amongst the elderly. PACE is a program most commonly available to the elderly poor. The demographic indicators can affect demand for services, size of the program, and the cultural sensitivities required in the provisions of services. Thus, these indicators provide variation within cultural-cognitive, normative, and policy-regulative elements, and sites that were selected represented those states with high, medium and low concentration of these population characteristics.

State position on the Affordable Care Act (ACA). As discussed in Chapter Two, the current health reform efforts are going to affect many aspects of health care payment and delivery of service. The Kaiser Family Foundation (KFF, 2012) summarized the states' official positions on the ACA legislation. For this study, support of the ACA reflects the states' progressive nature toward health policy. The states were organized into four categories: challenging, supporting, challenging and supporting, and no position (KFF, 2012). States that challenged the legislation contested the constitutionality of the individual mandate. All 25 states that challenged (except Virginia) also contested the constitutionality of the Medicaid expansion (KFF, 2012). All of the 11 states that supported the ACA backed the constitutionality of both the individual mandate and the Medicaid expansion. Two states that challenged and supported the ACA show a discontinuity in the state government's opinion, as the state's governor and attorney general took opposite positions in the litigation. The Kaiser Family Foundation 
categorized states as having "no position" (12) showing that the state in no way participated in the ACA litigation. This positioning was reflected in the culturalcognitive, normative, and policy-regulative elements of context.

Application of criteria to context elements. Table 3.3 summarizes the selection criteria and links the criteria to the elements. The cultural-cognitive element is reflected in the years of operation, demographic criteria and the state position on the ACA. The normative element is represented by PACE certification, overall LTSS system performance, and state position on the ACA. The policy-regulative element is observed through PACE certification, organizational form, overall LTSS system performance, and percentage of elderly living in poverty. The procedural element will be reflected through the organizational form and the overall LTSS system performance measure. The relational element will be represented by the percentage of minority elders and the overall LTSS system performance.

All PACE programs certified by CMS are required to use a capitated payment jointly funded by Medicare and Medicaid; assume all financial risk; and provide care using the prescribed interdisciplinary team care model. There are 89 PACE programs in the United States currently; however each has a different state-level and possibly local contextual elements. Two elimination criteria were applied to reduce the number of sites from 89 to 40 . Variation and selected sites were analyzed to represent each organization form, each quartile of LTSS performance, with a selection of state with high, medium and low concentrations of elderly demographic criteria, and representative of states 
supporting, challenging or having no position for the ACA. Table 3.4 provides a summary of the priority order in the sites that were recruited for this study. 
Table 3.3: Selection criteria links to context elements

\begin{tabular}{|c|c|c|c|}
\hline $\begin{array}{l}\text { Criteria } \\
\text { Purpose }\end{array}$ & $\begin{array}{l}\text { Selection } \\
\text { Criteria }\end{array}$ & Source & Context Element \\
\hline \multirow[t]{2}{*}{ Elimination } & $\begin{array}{l}\text { PACE } \\
\text { certified } \\
\text { program }\end{array}$ & $\begin{array}{l}\text { The National PACE association, } \\
\text { http://www.npaonline.org }\end{array}$ & $\begin{array}{l}\text { - } \text { Normative } \\
\text { - Policy-Regulative }\end{array}$ \\
\hline & $\begin{array}{l}\text { At least } 5 \\
\text { years of } \\
\text { operations }\end{array}$ & $\begin{array}{l}\text { The National PACE association, } \\
\text { http://www.npaonline.org }\end{array}$ & $\begin{array}{ll}\text { - } & \text { Cultural- } \\
& \text { Cognitive } \\
\text { - } & \text { Normative } \\
\end{array}$ \\
\hline \multirow[t]{6}{*}{ Variation } & $\begin{array}{l}\text { Organizational } \\
\text { form }\end{array}$ & $\begin{array}{l}\text { Program website and the National PACE } \\
\text { Association, http://www.npaonline.org }\end{array}$ & $\begin{array}{l}\text { - Policy-Regulative } \\
\text { - Procedural }\end{array}$ \\
\hline & $\begin{array}{l}\text { Overall LTSS } \\
\text { system } \\
\text { performance }\end{array}$ & $\begin{array}{l}\text { Reinhard, S. C., Kassner, E., Houser, A., } \\
\text { and Mollica, R. (2011). Raising } \\
\text { Expectations: A State Scorecard on } \\
\text { Long-Term Services and Supports for } \\
\text { Older Adults, People with Physical } \\
\text { Disabilities, and Family Caregivers. } \\
\text { Retrieved from: } \\
\text { http://www.longtermscorecard.org }\end{array}$ & $\begin{array}{ll}\text { - } & \text { Cultural- } \\
& \text { Cognitive } \\
\text { - } & \text { Normative } \\
\text { - Policy-Regulative } \\
\text { - Procedural }\end{array}$ \\
\hline & $\begin{array}{l}\text { Percent of } \\
\text { population } \\
\text { over the age of } \\
65\end{array}$ & $\begin{array}{l}\text { United States Census Bureau, 2010, } \\
\text { http://quickfacts.census.gov/qfd/index.ht } \\
\underline{\mathrm{ml}}\end{array}$ & $\begin{array}{ll}- & \text { Cultural- } \\
& \text { Cognitive }\end{array}$ \\
\hline & $\begin{array}{l}\text { Percent of } \\
\text { minority } \\
\text { persons over } \\
\text { the age of } 65\end{array}$ & $\begin{array}{l}\text { Administration on Aging, } 2010 \\
\text { http://www.aoa.gov/AoARoot/Aging St } \\
\text { atistics/Census_Population/census2010/I } \\
\text { ndex.aspx }\end{array}$ & $\begin{array}{ll}\text { - } & \text { Cultural- } \\
\text { Cognitive } \\
\text { - } \\
\text { Relational }\end{array}$ \\
\hline & $\begin{array}{l}\text { Percent } \\
\text { elderly } \\
\text { population } \\
\text { living in } \\
\text { poverty }\end{array}$ & $\begin{array}{l}\text { Kaiser State Health Facts, } \\
\text { Demographics and the Economy, } 2012 \\
\text { http://www.statehealthfacts.org/compare } \\
\text { cat.jsp?cat=1\&rgn=6\&rgn=1 }\end{array}$ & - Policy-Regulative \\
\hline & $\begin{array}{l}\text { State position } \\
\text { on ACA }\end{array}$ & $\begin{array}{l}\text { Kaiser State Health Facts, Health } \\
\text { Reform, } 2012 \\
\text { http://www.statehealthfacts.org/healthref } \\
\text { ormsource.jsp }\end{array}$ & $\begin{array}{l}\text { - } \text { Cultural- } \\
\text { Cognitive } \\
\text { - Normative } \\
\text { - } \text { Policy-Regulative }\end{array}$ \\
\hline
\end{tabular}


Table 3.4: Site selection criteria

\begin{tabular}{|c|c|c|c|c|c|c|c|}
\hline $\begin{array}{c}\text { PACE } \\
\text { Case } \\
\text { ID }\end{array}$ & $\begin{array}{l}\text { Years of } \\
\text { Operation, } \\
2012\end{array}$ & $\begin{array}{l}\text { Org. } \\
\text { Form, } \\
2012\end{array}$ & $\begin{array}{l}\text { Overall } \\
\text { LTSS } \\
\text { system } \\
\text { performance } \\
\text { by quartile, } \\
2011\end{array}$ & $\begin{array}{l}\% \text { Pop. } 65 \\
\text { and over } \\
\text { (by city), } \\
2010\end{array}$ & $\begin{array}{l}\% \text { Minority } \\
\text { Persons } \\
\text { Over } 65 \\
\text { (by state) }\end{array}$ & $\begin{array}{l}\% \text { Elderly } \\
\text { pop. living } \\
\text { in poverty } \\
\text { (by state), } \\
2010\end{array}$ & $\begin{array}{l}\text { State } \\
\text { position } \\
\text { on ACA, } \\
2012\end{array}$ \\
\hline 1 & 18 & $\begin{array}{l}\text { Health } \\
\text { System }\end{array}$ & 2 & 13 & 18 & 38 & Support \\
\hline 2 & 5 & $\begin{array}{c}\text { LTC } \\
\text { Provider }\end{array}$ & 1 & 14 & 9 & 9 & Challenge \\
\hline 3 & 14 & $\begin{array}{c}\text { LTC } \\
\text { Provider }\end{array}$ & 2 & 12 & 16 & 39 & Support \\
\hline 4 & 22 & $\begin{array}{l}\text { Health } \\
\text { System }\end{array}$ & 1 & 11 & 11 & 7 & Support \\
\hline 5 & 20 & $\begin{array}{c}\text { Free- } \\
\text { standing }\end{array}$ & 3 & 11 & 17 & 32 & Challenge \\
\hline 6 & 13 & $\begin{array}{c}\text { Free- } \\
\text { standing }\end{array}$ & 3 & 14 & 13 & 10 & Challenge \\
\hline 7 & 20 & $\begin{array}{c}\text { Free- } \\
\text { standing }\end{array}$ & 2 & 11 & 18 & 38 & Support \\
\hline 8 & 22 & $\begin{array}{c}\text { Free- } \\
\text { standing }\end{array}$ & 3 & 10 & 10 & 10 & Support \\
\hline 9 & 14 & $\begin{array}{l}\text { Health } \\
\text { System }\end{array}$ & 3 & 12 & 13 & 10 & Challenge \\
\hline 10 & 14 & $\begin{array}{l}\text { Health } \\
\text { System }\end{array}$ & 4 & 15 & 14 & 12 & $\begin{array}{c}\text { No } \\
\text { Position }\end{array}$ \\
\hline 11 & 22 & $\begin{array}{c}\text { LTC } \\
\text { Provider }\end{array}$ & 1 & 9 & 9 & 6 & Challenge \\
\hline 12 & 16 & $\begin{array}{l}\text { Health } \\
\text { System }\end{array}$ & 2 & 11 & 18 & 38 & Support \\
\hline 13 & 21 & $\begin{array}{c}\text { LTC } \\
\text { Provider }\end{array}$ & 1 & 10 & 12 & 16 & Challenge \\
\hline 14 & 5 & $\begin{array}{c}\text { Free- } \\
\text { standing }\end{array}$ & 4 & 11 & 19 & 27 & Challenge \\
\hline
\end{tabular}




\begin{tabular}{|c|c|c|c|c|c|c|c|}
\hline 15 & 16 & $\begin{array}{c}\text { Free- } \\
\text { standing }\end{array}$ & 3 & 10 & 10 & 10 & Support \\
\hline 16 & 6 & $\begin{array}{c}\text { Free- } \\
\text { standing }\end{array}$ & 3 & 11 & 10 & 13 & Challenge \\
\hline 17 & 12 & $\begin{array}{l}\text { Health } \\
\text { System }\end{array}$ & 1 & 11 & 9 & 10 & $\begin{array}{c}\text { No } \\
\text { Position }\end{array}$ \\
\hline 18 & 20 & $\begin{array}{c}\text { LTC } \\
\text { Provider }\end{array}$ & 4 & 14 & 18 & 27 & Support \\
\hline 19 & 15 & $\begin{array}{l}\text { Health } \\
\text { System }\end{array}$ & 3 & 11 & 10 & 11 & Challenge \\
\hline 20 & 8 & $\begin{array}{c}\text { Free- } \\
\text { standing }\end{array}$ & 3 & 12 & 17 & 32 & Challenge \\
\hline 21 & 17 & $\begin{array}{l}\text { Health } \\
\text { System }\end{array}$ & 1 & 11 & 12 & 12 & $\begin{array}{c}\text { Challenge } \\
\text { and } \\
\text { Support }\end{array}$ \\
\hline 22 & 29 & $\begin{array}{c}\text { Free- } \\
\text { standing }\end{array}$ & 2 & 11 & 18 & 38 & Support \\
\hline 23 & 10 & $\begin{array}{l}\text { Health } \\
\text { System }\end{array}$ & 1 & 12 & 9 & 9 & Challenge \\
\hline 24 & 17 & $\begin{array}{l}\text { Health } \\
\text { System }\end{array}$ & 3 & 10 & 10 & 10 & Support \\
\hline 25 & 17 & $\begin{array}{l}\text { Freestand } \\
\text { ing }\end{array}$ & 3 & 11 & 10 & 10 & Support \\
\hline 26 & 17 & $\begin{array}{c}\text { Free- } \\
\text { standing }\end{array}$ & 3 & 12 & 10 & 10 & Support \\
\hline 27 & 16 & $\begin{array}{l}\text { Health } \\
\text { System }\end{array}$ & 2 & 12 & 14 & 29 & Support \\
\hline 28 & 17 & $\begin{array}{l}\text { Health } \\
\text { System }\end{array}$ & 3 & 12 & 10 & 13 & Challenge \\
\hline 29 & 20 & $\begin{array}{l}\text { Health } \\
\text { System }\end{array}$ & 4 & 9 & 18 & 27 & Support \\
\hline 30 & 16 & $\begin{array}{l}\text { Health } \\
\text { System }\end{array}$ & 4 & 11 & 18 & 27 & Support \\
\hline 31 & 15 & $\begin{array}{c}\text { Free- } \\
\text { standing }\end{array}$ & 4 & 11 & 18 & 27 & Support \\
\hline
\end{tabular}




\begin{tabular}{|c|c|c|c|c|c|c|c|}
\hline 32 & 15 & $\begin{array}{c}\text { Free- } \\
\text { standing }\end{array}$ & 3 & 14 & 10 & 11 & Challenge \\
\hline 33 & 14 & $\begin{array}{c}\text { Free- } \\
\text { standing }\end{array}$ & 3 & 12 & 13 & 10 & Challenge \\
\hline 34 & 5 & $\begin{array}{c}\text { Free- } \\
\text { standing }\end{array}$ & 3 & 12 & 13 & 10 & Challenge \\
\hline 35 & 12 & $\begin{array}{c}\text { Free- } \\
\text { standing }\end{array}$ & 3 & 14 & 13 & 10 & Challenge \\
\hline 36 & 22 & $\begin{array}{l}\text { Health } \\
\text { System }\end{array}$ & 3 & 9 & 13 & 22 & Challenge \\
\hline
\end{tabular}

Sources: Kaiser State Health Facts, 2012; National PACE Association, 2012; Reinhard, Houser, \& Mollica, 2011; U.S. Census Bureau, 2012 


\section{Research Rationale and Design}

A qualitative case study is an approach to research that facilitates exploration of a program, intervention, or phenomenon in its particular context. The research question will be explored through multiple lenses, allowing the perspectives of administrators and a broad spectrum of IDT members to be heard. Approaches developed by both Stake (2006) and Yin (2009) will be used here. Their case study approaches are built on a constructivist paradigm, meaning that truths are relative and created through the interaction of people in a given setting, and influenced by a combination of history, culture, and biography. Qualitative research allows participants to describe the nature of reality from their own perspective, in their own words rather than using theories or variables determined by the researcher. Multiple case studies enable the researcher to explore differences within and between cases. Cases are selected carefully, so as to assure the researcher will observe variations in the institutional context, and the affect they may have on the functioning of the IDT.

Yin (2009) states that a case study should be considered when: (1) manipulation of the behavior of those involved is not possible; (2) one is trying to understand the contextual conditions because they are relevant in answering the research question; and (3) there are unclear boundaries between the phenomenon and the context. Yin (2009) and Stake (2006) emphasize the importance of defining the scope for each case. Defining the criteria will keep the question in focus and prevent the researcher from trying to do too much. 
The qualitative research design begins with questions, which help guide the research but are not intended to confine the respondent and other data collection. Core theoretical concepts have been will be identified and linkages will be developed among the core concepts and the data (Heath 2004). This process will lead to identification of core concepts that are central to the research question.

To ensure the integrity of the data and data collection process, the role of the researcher was clearly outlined to participants, and management of the contact with institutional representatives was carefully considered; steps were taken to protect the interests of the participating sites and their representatives. To ensure the integrity of the research process, multiple interviewees were used from each site; measures were introduced to ensure rigor of data recording and subsequent analysis; and additional measures were implemented to enhance the trustworthiness of the research. Figure 3.2 provides an overview of the research design logic, from the problem being addressed to how this research aimed to address it.

\section{Human Subjects Protection}

A human subjects research application was submitted to the Portland State University Human Subjects Research Review Committee (HSRRC), and received approval on October 19, 2012 (HSRRC Proposal \#122337). The application and approval was shared with participating sites. Confidentiality was a special consideration; there are only seven cases in this study. Organizations and the participants are not identified. Consent forms were provided to all key informants, which informed them of their rights as a research participant and provided contact information for questions or 
concerns. Consent was obtained verbally prior to each interview and a physical copy of the consent form was mailed to the participant after the completion of the interview. All interviews were digitally recorded and were transcribed, verbatim, for analysis. 
Figure 3.2: Overview of Research Design Logic

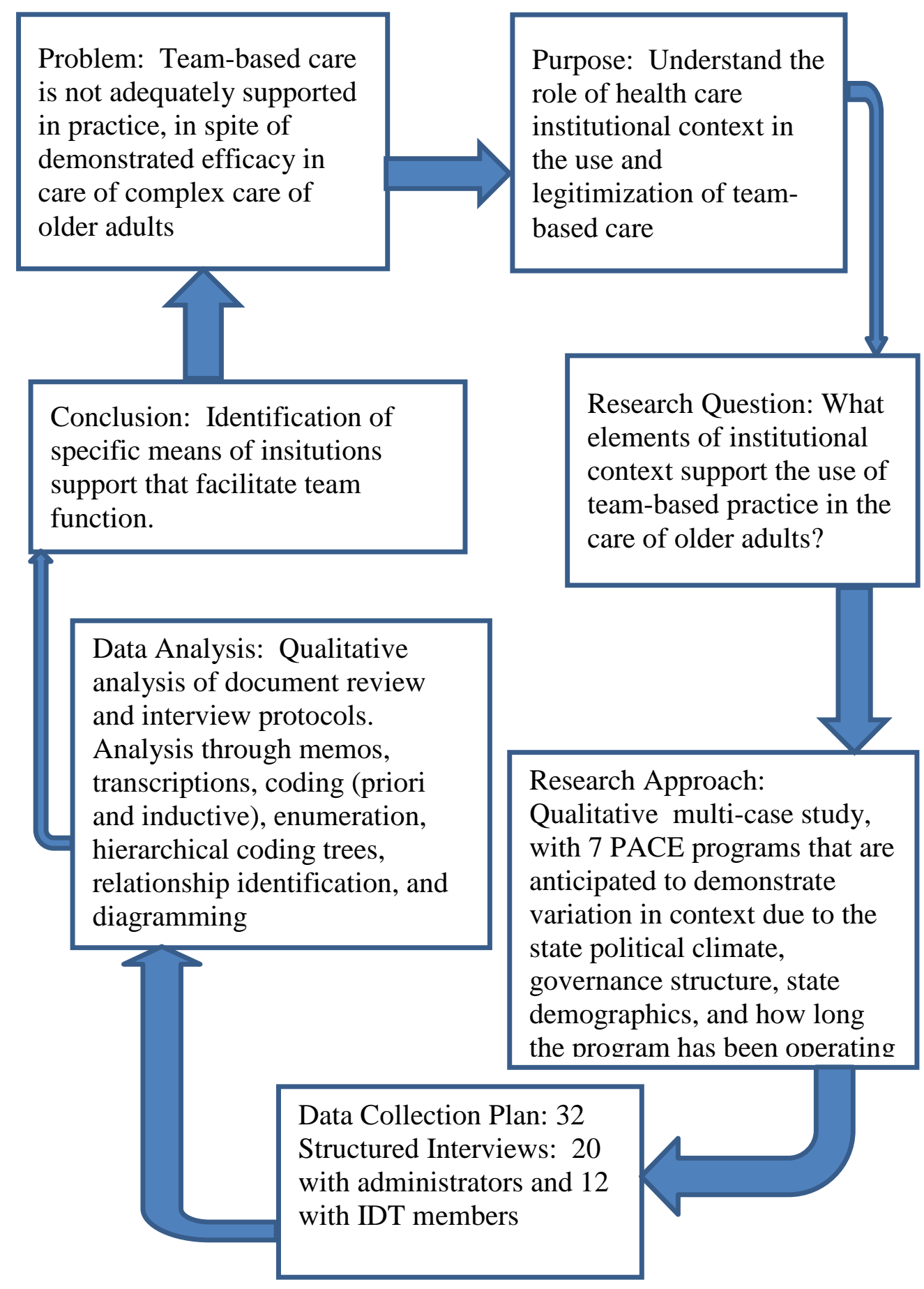


There were no physical or psychological risks to the interviewees. There was a cost in terms of time to those participating. Interviews lasted between 45 and 96 minutes. The researcher coded all interviews; the codes were used in notes as well as in transcription. The use of the data is for this dissertation and subsequent journal articles or conference papers. Only the dissertation committee members and the researcher knew the identities of the organizations and research participants. Dissertation committee members did not hear nor have any access to the recordings or the raw transcripts, which helped preserve the confidentiality of responses. Participation in this study could contribute to the growing body of literature relevant to interdisciplinary teamwork. This study provides a deeper understanding of the role of institutional context in providing adequate support to facilitate interdisciplinary team care delivery.

\section{Role of the Researcher}

The individual researcher conducting this dissertation was the only interviewer, coder, and analyst of the data. This enabled a level of consistency in interview style, coding technique, and analysis. The researcher highlighted how the PACE program is formally structured to support the interdisciplinary teams from different points of view. A single coder limits the perspective and interpretation of the data; however, time and resource constraints limited the addition of other coders. In addition, as a dissertation, the expectation is that the research was done independently and not by a team.

\section{Data Collection}

Data collection occurred from November 2012 through February 2013. Seven PACE programs participated, and they represented six states. Thirty-two interviews were 
conducted -- 20 with administrators and 12 with IDT members. The following section briefly describes the sites and participants, while maintaining confidentiality parameters. Many descriptors needed to be broadened or removed in order to assure that the organizations and individuals could not be identified. Three of the seven sites expressed concerns about confidentiality; the approach for reporting was modified in order to assure that site and participant identities would remain confidential. This new approach moved away from a multi-case study as originally proposed to an aggregated case study focused around the institutional elements. The purposive sample of this study was selected based on the four selection criteria for variation. The analysis focused on what supports were present and considered significant for all sites regardless of variation in context.

The sites were recruited from October 2012 to January 2013. There was an initial effort to recruit the top five organizations identified by the selection criteria. Four of those five organizations agreed to participate; five more PACE sites were contacted in order to expedite recruitment of the final site. From the second recruitment effort, three more sites were recruited. As a result, a decision was made to include all seven sites (as compared to the five originally intended). Thirty-two interviews were conducted between November 2012 and April 2013. Most interviews were conducted via phone/Skype, and all interviewees agreed to be audio-recorded. Interviews ranged in length from 48-123 minutes. The researcher transcribed each interview verbatim. Each transcript was reviewed and coded at least twice in order to identify codes, sub-codes, and unexpected categories that may have been missed during the initial coding. 
Sites and participants were randomly assigned alphanumeric codes to maintain the confidentiality of sites and individuals interviewed. Each quote in the following chapter is labeled by a citation that identifies if the quote came from an administrator or IDT member, as well as an alphanumeric code to assure quote representation from different interviews. Table 3.5 provides the counts of administrators and IDT members interviewed at each site.

Table 3.5: Categories of interviewees

\begin{tabular}{c|c|c|c}
\hline Case ID & Administrators & IDT Team Members & $\begin{array}{c}\text { Total number of } \\
\text { interviews }\end{array}$ \\
\hline 1 & 2 & 1 & 3 \\
\hline 2 & 5 & 2 & 7 \\
\hline 3 & 2 & 1 & 3 \\
\hline 4 & 3 & 3 & 6 \\
\hline 5 & 3 & 3 & 6 \\
\hline 6 & 2 & 0 & 2 \\
\hline 7 & 3 & 2 & 5 \\
\hline Total & 20 & 12 & 32 \\
\hline
\end{tabular}

The sites selected had all been in operation for over 10 years, except for one that had been operating between five and 10 years. All three organizational structures described in the previous section were represented by more than one site. Two participating sites are part of an integrated health system. Two were freestanding PACE programs with no larger parent organization. Three programs were part of a larger longterm care organization. The state long-term services and supports (LTSS) rating (Reinhard, Kassner, Houser \& Mollica, 2011) showed that six of the seven sites were in the top two quartiles of the rating, with only one participating site in the third quartile. The proportion of the state population over 65 in the states represented by these 
organizations ranged from 9 to 15 percent. Finally, there was a variation in level of support for the Accountable Care Act (ACA) with four of the sites in states that supported the ACA and three in states that opposed it (Kaiser Family Foundation, 2012).

Each program was invited to participate in the study through its organizational gatekeeper, which varied per site. Key informants were selected based on management level; one individual was selected per level to provide a different perspective on the context. Interviews utilized a structured interview protocol (see Appendix A and B) that inquired about the institutional governing structures relevant to interdisciplinary team support.

Table 3.6 links the questions in the interview protocol to the five context elements in this study's conceptual framework (Figure 3.1). 
Table 3.6: Interview protocol question links to context elements

\begin{tabular}{|c|c|}
\hline $\begin{array}{l}\text { Context } \\
\text { Element }\end{array}$ & Protocol Question \\
\hline $\begin{array}{l}\text { General } \\
\text { Opening }\end{array}$ & $\begin{array}{l}\text { 1. Please describe the goals and mission of your organization. } \\
\text { 2. What is your role/relationship to the PACE interdisciplinary } \\
\text { (IDT) team? }\end{array}$ \\
\hline $\begin{array}{l}\text { Cultural- } \\
\text { Cognitive }\end{array}$ & $\begin{array}{l}\text { 3. From your point of view, in what ways is the IDT culture within } \\
\text { the PACE program similar to the organizational culture? In what } \\
\text { ways is it different? Please explain. } \\
\text { 4. What are the common beliefs, shared logics or working patterns } \\
\text { amongst the IDT? } \\
\text { 5. Do you believe that the IDT culture is similar amongst PACE } \\
\text { programs nationwide? Tell me about that }\end{array}$ \\
\hline Normative & $\begin{array}{l}\text { 6. How does the IDT define their goals and objectives? How does } \\
\text { it select the appropriate way to pursue them? } \\
\text { 7. How is the approach to leadership decided within the team? } \\
\text { 8. Are there other professional boundaries that effect goal } \\
\text { accomplishment? } \\
\text { 9. What is the value added for your organization, in your opinion, } \\
\text { for the increasing the acceptance of team-based patient care, } \\
\text { since the opening of the PACE program? } \\
\text { 10. In what ways does the PACE model influence any other areas of } \\
\text { care delivery? }\end{array}$ \\
\hline $\begin{array}{l}\text { Policy- } \\
\text { Regulative }\end{array}$ & $\begin{array}{l}\text { 11. How has your organization been able to work within its policy } \\
\text { and regulatory framework to affect IDT functioning? } \\
\text { 12. Does your state or organization utilize waivers to change the } \\
\text { scope of PACE services? } \\
\text { 13. How are responses developed to issues that arise in relation to } \\
\text { care quality and team function? }\end{array}$ \\
\hline Procedural & $\begin{array}{l}\text { 14. What organizational characteristics have influenced the time and } \\
\text { space that IDTs have to meet and collaborate in decision-making } \\
\text { around patient care? } \\
\text { 15. Are daily routines formalized? How much so? } \\
\text { 16. Do issues of urgency and complexity of tasks interfere with team } \\
\text { functioning? }\end{array}$ \\
\hline Relational & $\begin{array}{l}\text { 17. How does your organization minimize the potential for conflict } \\
\text { between professions within the IDT? } \\
\text { 18. How do you measure successful team function within your } \\
\text { organization? } \\
\text { 19. What are the continuing education opportunities for the } \\
\text { organization about team working? Education opportunities for } \\
\text { the entire team? Opportunities for individuals? } \\
\text { 20. Some programs use ongoing team or organizational } \\
\text { development activities designed to improve team functioning. } \\
\text { Does your program do this? }\end{array}$ \\
\hline
\end{tabular}




\section{Data Analysis}

After the raw data were collected, there were seven steps in the analysis of the data, as shown in Table 3.7.

Table 3.7: Qualitative analysis steps and definitions

\begin{tabular}{l|l}
\hline Concept & Definition \\
\hline Memo & $\begin{array}{l}\text { Reflective notes were taken throughout the data collection } \\
\text { process }\end{array}$ \\
\hline Transcription & Verbatim records taken from the recorded audio files \\
\hline A priori codes & $\begin{array}{l}\text { Identification and definition of codes was done prior to data } \\
\text { analysis. Anticipated priori codes include positive and } \\
\text { negative categorizations, Table 3.3 indicators, and Table 3.7 } \\
\text { codes. }\end{array}$ \\
\hline Inductive Coding & Codes were created during the analysis \\
\hline Enumeration & $\begin{array}{l}\text { The quantifying of qualitative data, used strictly to identify } \\
\text { trends and collective perceptions }\end{array}$ \\
\hline Hierarchical coding trees & $\begin{array}{l}\text { Nesting of related concepts together from general concepts to } \\
\text { more specific ideas }\end{array}$ \\
\hline Relationships and diagrams & $\begin{array}{l}\text { Create a graphic that communicates how concepts are } \\
\text { associated or how they work. }\end{array}$ \\
\hline
\end{tabular}

The overall strategy for data analysis was based on the framework derived from institutional and team theory as noted in the previous chapters and summarized in Table 3.2. Coding schemes were developed cumulatively, and resulted in creation of a matrix for organizing the data. The data were organized by institutional element and aggregated to maintain organizational confidentiality. Generalizations about the institutional context and its role in supporting interdisciplinary teams in the care of older adults were derived by identifying the categories used most commonly in at least 15 unique interviews. Table 3.8 illustrates the priori coding schemes for each contextual element. 
Table 3.8: Relationship among contextual elements, institutional structure and coding

\begin{tabular}{|c|c|c|}
\hline $\begin{array}{l}\text { Contextual Elements } \\
\text { (Scott, 2008a; Reeves, } \\
2010)\end{array}$ & Institutional Structure & $\begin{array}{l}\text { Examples of Priori Codes } \\
\text { (Ducanis \& Golin, 1979; Yeatts \& Hyten } \\
\text { 1998) }\end{array}$ \\
\hline $\begin{array}{ll}\text { - } & \text { Normative } \\
\text { - } & \text { Regulative }\end{array}$ & Federal & $\begin{array}{ll}\text { - } & \text { Medicare/Medicaid Policy } \\
\text { - } & \text { Accreditation/certification } \\
\text { - } & \text { Resource allocation } \\
\text { - } & \text { Education/training } \\
\end{array}$ \\
\hline $\begin{array}{ll}\text { - } & \text { Normative } \\
\text { - } & \text { Regulative }\end{array}$ & State & $\begin{array}{ll}\text { - } & \text { Policy } \\
\text { - } & \text { Licensing } \\
\text { - } & \text { Policy agenda } \\
\text { - } & \text { Resource allocation } \\
\text { - Unions/professional organizations } \\
\text { - }\end{array}$ \\
\hline $\begin{array}{ll}- & \text { Normative } \\
- & \text { Regulative } \\
- & \text { Cognitive- } \\
& \text { Cultural }\end{array}$ & $\begin{array}{l}\text { Health Services } \\
\text { Organization }\end{array}$ & $\begin{array}{ll}\text { - } & \text { Mission/philosophy } \\
\text { - } & \text { Values } \\
\text { - } & \text { Policy } \\
\text { - } & \text { Resource allocation } \\
\text { - } & \text { Rewards/benefits } \\
\text { - } & \text { Personnel evaluation } \\
\text { - } & \text { Union agreements } \\
\end{array}$ \\
\hline $\begin{array}{ll}\text { - } & \text { Normative } \\
\text { - } & \text { Regulative } \\
\text { - } & \text { Cognitive- } \\
& \text { Cultural } \\
\text { - } & \text { Relational } \\
\text { - } & \text { Procedural }\end{array}$ & Program & $\begin{array}{l}\text { - } \text { Mission/philosophy/values } \\
\text { - Union agreements } \\
\text { - } \text { Culture } \\
\text { - } \text { Protection of team time } \\
\text { - } \text { Physical location } \\
\text { - } \text { Partnerships/contracts } \\
\text { - } \text { Client load } \\
\text { - } \text { Putside responsibilities } \\
\text { - } \text { Continuing education/training } \\
\text { - } \quad \text { Demographics/geography } \\
\end{array}$ \\
\hline $\begin{array}{ll}\text { - } & \text { Normative } \\
\text { - } & \text { Cognitive- } \\
& \text { Cultural } \\
\text { - } & \text { Relational } \\
\text { - } & \text { Procedural }\end{array}$ & $\begin{array}{l}\text { Interdisciplinary } \\
\text { Teams }\end{array}$ & $\begin{array}{ll}\text { - } & \text { Culture } \\
\text { - } & \text { Demographics/geography } \\
\text { - } & \text { Discipline diversity } \\
\text { - } & \text { Locations/meetings/spaces } \\
\text { - } & \text { Participation in team activities } \\
\text { - } & \text { Education/training cross-discipline } \\
\text { - Union } \\
\text { - }\end{array}$ \\
\hline
\end{tabular}


The analysis was dissected, managed, and displayed using NVivo10 and Microsoft Word. NVivo10 qualitative software by QSR International (2012) was used to manage all qualitative data. Previous experience by the researcher with NVivo10 software enabled it to be an asset to this research in the following ways: it allowed importation of data from multiple origins; interview transcripts were analyzed directly in NVivo10; and most importantly, it allowed the researcher to work efficiently with large volumes of data.

\section{Trustworthiness of Research}

Just as quantitative research methods must stand up to the statistical rigors of validity and reliability, qualitative research must also be subject to a set of rigorous criteria to defend the trustworthiness of the research. These criteria include credibility, transferability, dependability, and confirmability (Gobi \& Lincoln, 2005; Marshall \& Rossman, 1999). The study met the credibility criterion by adequately identifying and describing the subject of the study and the selection process for cases (Marshall \& Rossman, 1999). The selection process was derived from a theoretical framework, based on the literature review in Chapter Two. The second criterion, transferability, ensures that the findings will be useful to others in similar situations, with similar research questions or questions of practice (Marshall \& Rossman, 1999). Transferability is primarily ensured by the use of multiple data sources. Multiple sources created a foundation for the study through an examination of previous research and literature that presented a theoretical basis for the issues being studied. Using multiple sources within one site provided that more than one data source presented evidence to support a 
conclusion. The third criterion, dependability, is the methodological description that will allow this study to be repeated. This research study remained transparent, flexible, and as unbiased as possible as it progressed. The fourth and final criterion for trustworthiness is confirmability. Confirmability was accomplished through documentation, organization, creation of matrices, and validation of data by key informants.

\section{Limitations}

No single study can do everything, or even do all of the things it tries to do as completely as one would like. Anticipated limitations of this study included the fact that

analysis and coding were conducted by one coder (the researcher), which may reduce the objectivity in the coding. However, given that this was an individual dissertation, it would not be appropriate in this context to use another coder. Generalizability of results may be problematic, which is inherent to the case-study methodology. The final limitation was the limited time and resources to complete this work within a reasonable period; this was expected given that this was unfunded dissertation research.

\section{Conclusion}

Institutional context may facilitate or create obstacles in the maintenance of the PACE IDT within an organization. Through a qualitative multi-case study research design, seven PACE sites were recruited and 32 interviews were conducted to identify the specific characteristics of institutional context elements that support teams in caring for older adults. 


\section{Chapter 4 Presentation and Analysis of Data}

\section{Introduction}

This study was designed to investigate the influence of institutional context in the support and legitimization of interdisciplinary teams in the care of older adults. The PACE programs provide a standardized, team-based model of practice that allows for variation in context to be observed. The seven PACE organizations selected for this study represented a diversity of state policies, local cultures, normative organizational structures and procedures, and relationships. The results were derived from thirty-two interviews with PACE employees.

The study was designed to explore the institutional variation in the utilization and legitimacy of interdisciplinary teams in the care of frail older adults who have chronic physical, mental, and/or cognitive diagnoses. Given the complicated and multi-faceted nature of health care institutions in the U.S., and the lack of previous research addressing this question, this study offers a first step in understanding the context surrounding how teams are legitimized and how variation in context affects IDT support. This study used a theoretical framework based on the literature review that identified five elements of institutional contexts: policy-regulative, cultural-cognitive, normative, relational, and procedural.

As described in Chapter Three, the sampling selection criteria were developed based on relevance to the research question, providing diversity across contexts and opportunities to learn about complexity and context. Seven PACE organizations 
participated; three to seven interviews were conducted with each site. Twenty administrators and 12 IDT members were interviewed. The original intent was to include external governing board members in the interviews, but access to such individuals was not provided, and so no interviews with these individuals were possible. PACE organizational leaders did not feel that the board members could commit the $60-90$ minutes to do the interview.

The discussion of findings in this chapter is organized by institutional element, starting with those affecting the broadest macro institutions (Policy-Regulative), then moving through the more organizational focused elements (Normative, CulturalCognitive) to the micro institutions at the program and team level (Relational and Procedural). Categories emerged through the analysis based upon the frequency with which they appeared in interviews. At least 15 unique interviewees cited all categories within each element, described below. Representative quotes were selected that best captured the sentiment expressed by the interviewees. Interviewees were asked, through a series of questions, to reflect on the institutional context characteristics that influence the functioning and behavior of the IDTs in providing care for older adults.

\section{Policy-Regulative Elements}

To review, the policy-regulative element speaks to the respective institutions' rule-setting, monitoring, and sanctioning activities. As described by Scott (2008a), the processes involved include the capacity and authority to establish rules inspect and judge others' conformity to those rules, and when necessary manipulate sanctions in order to influence future behavior. Teams have competing interests, which requires mediation by 
rules and referees to ensure order. Even though the team is pursuing a similar set of goals, the individuals are on some level pursuing their collective self-interest (DiMaggio $\&$ Powell, 1983) or in this case the interest of the PACE enrollee. The third parties must be neutral or the outcomes will be influenced by their behavior and may not serve the intended end (North, 1990). Many rules and laws are ambiguous enough that, contrary to common belief, there may not be a clear code of conduct. The interviews highlighted three categories that affect IDT's support: actions of regulatory bodies, resource allocation decisions, and quality measurement.

Regulatory bodies. PACE has a unique history and its relationship with Medicare and Medicaid payment structures, including the capitated payment system, creates a complex regulatory structure with multiple stakeholders. The ACA has provided new attention to PACE, and how it can be expanded to serve more people. Federally, CMS has expressed a level of urgency to expand the PACE model to meet the growing needs of an aging population. An administrator paraphrased a recent meeting held with CMS officials as follows:

While [the PACE program nationwide] serves 25, 000 people across the country, [CMS] has 9 million people to worry about. If you can't scale up, I'm here to tell you, you will not be part of our solution in the future.' It is a little disingenuous for [CMS], because on the one hand, they want us to scale up, but on the other hand, they are very inflexible about the regulations (PACE Administrator, C4). 
The PACE regulations have been revisited in an effort to consolidate regulatory interests; however, there continue to be multiple regulatory bodies that require reports. Specifically, some Medicare and Medicaid offices are attempting to reduce redundancy between their respective regulatory activities. PACE staff, complain that they spend significant time fulfilling regulatory documentation, resulting in less time with participants.

[The state has] asked us how can we scale up. They don't have the money for this, but their question was how could we be available in every single county in the state? Our flat answer was "could you reduce the bureaucracy and the number of people who survey us." We are persistently surveyed, it's ridiculous. It's the Department of Health to the kitchen, Department of Health for home health, Department of Aging for the day care, the CMS people, the state protective services, the state Department of Aging. It never stops. It ties your hands and one regulation might in fact conflict with another, but the other one supersedes, so you think you've figured it out but you're wrong. It's one of the most important things for the PACE programs, nationally and in the state (PACE Administrator, G6).

Program administrators emphasized repeatedly the importance for the PACE program to grow and scale up in order for it to survive as a care and payer model. Overall regulation of PACE was expressed as disproportionate to the number of individuals 
served compared with other health care providers. One administrator compared the JCAHO accreditation to the average PACE CMS audit:

PACE is an amazingly regulatory driven program. I would say more so than a nursing home or hospital. The amount of regulation and oversight that we have relative to our number of patients is phenomenal. You go to a major metropolitan hospital, JCAHO goes in there for 2-3 days, and they've seen tens of thousands of patients. Our audit that we get, CMS comes in here with 4-5 auditors and sits here for an entire week analyzing our 350 patients. We've got federal regulations, and a set of state regulations, and then on top of that we've got a whole ream of CMS interpretation of those regulations. They've published things that while they might not be regulations, they might as well be, there is this huge document on their interpretation of care planning for example, which for all intents and purposes serves as a regulation for us, because we have to follow it. Then there are the different interpretations, broadly varying from what the regulations is, to whatever their interpretation of the regulation is. In that sense, it becomes a new regulation to us (PACE Administrator, D5).

The shortage of practitioners in all geriatric specialties and the difficulties in retaining paraprofessionals create opportunities for PACE teams to utilize alternative workforce models and configurations. However, PACE regulations mandate team composition, requiring eleven IDT members and that a physician 
must be the primary care provider. According to study participants, these requirements can inhibit PACE teams from taking full advantage of the team model. A significant burden is the commitment to a physician, and the disempowerment of nurse practitioners (NP) as primary care providers. PACE programs reported a minimizing of the NP roles in some states, where the NP actually experiences a reduced scope of practice within PACE compared with how the NP can practice autonomously outside of the PACE program given local definition of scope of practice.

One example I can give you is just with our nurse practitioners. It's a fairly narrow scope that the PACE regulations allow them to fulfill. Quite frankly here a nurse practitioner could go hang out a sign and have their own practice, they could have their own office. But within our model it's very prescriptive and the NP has to be supervised and signed-off by a physician and that tends to feel very constraining to them (PACE Administrator, E5).

PACE programs might not be able to use creative and possibly cost-saving solutions to common health issues experienced by the frail elderly because of Medicare/Medicaid funding requirements. The ongoing issue that PACE IDTs face is providing participant-centered care, with the focus on quality of life and independence, while simultaneously having to work within a highly medicalized payment and regulatory system. Interviewees remarked that the payment and regulatory system limits their 
ability to customize treatments specifically for a participant's home setting and conditions requiring intervention.

We wanted to try using a bidet with one of our residents in housing. They were having recurrent UTIs and we hit a roadblock that did not allow us to purchase the bidet because it wasn't a Medicare approved intervention. We were trying to be creative by using an approach that has worked in other settings in treating UTIs. It wasn't something that, as a Medicare healthcare provider, we could purchase because it wasn't clinically proven as an intervention. The bidet intervention is not unsafe; the studies just haven't been done yet to prove it is effective. There are definitely times where those things happen because we try to meet Medicare standard on things (PACE IDT Member, I2).

Administrators described efforts to protect IDT members from feeling the effects of the regulatory burden. However, a provider illustrated how the multiple regulators can affect the time for patient care:

I think [data entry] interferes and people are so busy thinking about which report they have to file, when it's due and when you have to close it. I think the amount of regulation detracts from care. I know it is supposed to improve quality, but I have a hard time seeing that. The one example that comes to mind for me is falls. It's laborious, PT keeps track of every fall, where it happened, what time it happened, was there an injury, was there 
an ER, so we're collecting a lot of data and it's all we'll have, so it's all on an excel spreadsheet and it's time consuming. We now have to fill out a safety record on every fall that occurs, we do them everywhere. If they're at home, wherever this happens so now we have to fill out another form. So our PT has five different places where they have to enter this stuff and none of them speak to the other. They're all separate (PACE Administrator, J1).

Administrators perceive that the PACE payment structure is a very bureaucratic burden on programs and some interviewees expressed a lack of coordination between federal CMS regulation and state Medicaid regulation. The lack of coordination also exposes a disconnect between regulatory expectations and regulatory reality, when regulators want 'scaling' of the PACE program, yet do not relax regulation correspondingly to allow that to happen. There is also a disconnect between the views of those who are regulated and those who regulate.

Resource allocation. The IDTs act like insurers in deciding on services to be provided to individuals, while staying aware of costs to the organization. Where policy and regulation can inhibit creative and unusual approaches, strict policies also allow the IDT to deflect responsibility of a service denial. When requests for a service are perceived as unreasonable or do not serve a direct purpose to care, the IDTs value being able to point to a policy to validate the choice to decline a service. As one administrator explained: 
Many people here really value having a policy that they can just go to and sort of lean on and make decisions easy and simple. I think the new policy changes are designed to be more overt in getting us to where we need to be as far as really identifying member outcomes and developing more discussion around natural supports and supports outside of [PACE] that can help meet a member's need. I think it is going to feel like the process is more involved, so you will get some pushback on that, but we'll see. It is always something (PACE Administrator, B4).

PACE organizations have their own organizational policies that guide the team members in differentiating between a participant's "needs" and "wants." It can be difficult for IDTs to discern the fine line, within a participant-centered program, between keeping a participant happy and indulging them. Many of these policies focus on the outcome of the service requested. Clear organizational policies remove any decision-making habits or shortcuts the IDT members may have used before or in other participant cases. Resource allocation policies require the IDT to think about how the service adds to the quality of life and the expected outcomes, and not default to participant wants and provider habits in health care decisions. As one IDT member explained:

Before we always had a culture of making sure that when a member makes a request it is something that they need, it had a particular outcome. [The policy] forced team members to really think and dig into member outcomes, is the service they're requesting something that they need or is 
it something that they want. It forces us to talk through more cost effective options or other options. As the rules and regulations change, as DHS and CMS begin to look at the sustainability of long-term care and bring down directives that we have to follow to look at cutting costs and still being able to provide still those positive member outcomes (PACE IDT Member, L4).

Many PACE participants request access to a motorized wheelchair. This issue came up in all but three interviews. The repetition of this issue reflects the larger issue of teams acting as insurers, to some extent, and meeting the needs of participants. Every team struggles with balancing the participant's needs and the participant's wants. Financial aspects are the jurisdiction of the administrators. The IDT may also consider cost or value, but overall the IDT focuses on providing all-inclusive care, and defining what that means.

We'd like to preserve their independence as much as we can. There's this fine line, do we pay $\$ 10,000$ [for a motorized wheelchair] so that they can have a little more independence, even though they're living in an assisted living facility where all of these things are supposed to be done for them. Once they get [the MW] maybe they'll stop trying to walk anymore and get more de-conditioned and weaker and even more dependent. So, there are all of these pros and cons and it's rarely an obvious answer (PACE IDT Member, M2). 
Resource allocation issues also arise with choices the team must make regarding dentures. More often than not, this request comes from IDT members or family on behalf of the participant. The IDT must weigh the necessity of dentures to the individual's quality of life. Half of the interviewees described participants losing their dentures, with those who have dementia and Alzheimer's disease, in particular, losing dentures on a relatively regular basis. Some team members believe that these participants are entitled to a set of dentures even though they may be lost frequently, while others see it as a waste of limited resources, especially if there are no health consequences in denying future sets of dentures. These different perspectives reflect norms, as described below:

For example, somebody who had never had dentures their entire life, actually doesn't have a ridge anymore in their mouth, requesting dentures. Somebody in the team saying, "Everybody deserves teeth." I'm saying, “Okay, there's no weight loss. There's no sort of any other, if you will, incredibly cosmetic reason why the person feels isolated and offended by their own look. They haven't had teeth for 25 years. They frankly forgot about they don't have teeth. Replacing the teeth, means we will be replacing them again soon (PACE Administrator, G6).

Organizational efforts to promote client quality of life and independence are persistent issues. Customizing care plans to each individual's needs may not always align with standardized quality markers. What is helpful to one participant may not be helpful to another. Due to the all-inclusive, participant- 
centered nature of PACE, many of the services and infrastructure are made possible by the capitated model.

Some of the services provided and the evolution of PACE is not based on experience of the PACE Program. You come up with $90 \%$ of the upper payment model and say they go ahead and give you $90 \%$ of X. There are certain things you can't quantify in numbers. I mean the experience of coming into an ADHC setting is just but one of the things. We approve some of those things as cleaning those people's homes when they have roaches. We do a lot of this. How do you put those into quantifiable numbers? Is it going to be one unit of doing this or is it going to be based on cost? This is a problem because we haven't come up with a way to articulate all of the elements that we have and quantify them because they're not easily quantifiable. The capitated model still doesn't seem to get the full services we provide because we build the infrastructure. We have the buildings that people come to. You're not going to be able to value those, but we're paying for those (PACE Administrator, N1).

Providing all-inclusive care for the elderly distorts how participants interact with their providers. Interviewees reported that PACE participants may be prone to moral hazard, as the all-inclusive health care they are receiving does not directly cost them anything. The focus of health care decisions can shift from what a participant needs to what they want. The onus falls on the IDT to 
agree to or decline such requests. Thus, there may be application of the "I want a pony rule" as described by this interviewee:

I think they thought patient centered meant it had to be $100 \%$ what the patient wants, which I started calling it the "I want a pony rule." I remember a social worker saying, "The patient says he wants to move to an independent setting or walk a mile." Well, this person is in a wheelchair, is never gonna walk again, it just ain't gonna happen. Wishing doesn't make it so, he's never gonna live independently (PACE IDT Member, M2).

Every state has a grievance process that allows participants to appeal when they disagree with a care decision or service denial. This process allows participants and their families to take issue with the care decisions made by the IDT. The number of grievances filed, and the state's level of satisfaction with the PACE program's handling of the grievances, may trigger an audit or review at the state level. Each state Medicaid office has a different approach to audits in term of intensity and frequency. Some coordinate with the CMS audit, and some conduct their own, which may be just as intense for the PACE program as a CMS audit.

They [the state] generally come out on the audits with CMS. Anyone that files a grievance with the state it generally goes to that program manager. The calls we tend to get from that program manager are, "I got a call from 
Mrs. Smith," here's her complaint, how are you dealing with it? That is the main oversight we see from the state, based on grievances or things that they hear are wrong. They don't come down and do individual audits of their own. We rarely get questions about that data we send in (PACE Administrator, D5).

Meaning of quality. "Some of the metrics that we are monitoring we're not reporting them in a way that's actually meaningful. We have data but no information" (PACE Administrator, C4). The health needs of the frail elderly should be patientcentered and comprehensive (IOM, 2008). The IOM reports (2008) that an individual's care needs should include prevention and coordination of chronic, acute, and social care health needs, and PACE programming responds to this. However, interviewees report that quality is still measured by acute care metrics. Interviewees' responses were varied in how well they believe these metrics adequately capture long-term quality of life and care as supported by an IDT.

Metrics that [the organization] use to measure the effectiveness of the teams, monitor ER visits, hospital rates and wounds. From what I understand, all this data measures how effective the team is. I know they take into consideration team meetings. They want the meetings to be succinct and to the point, interfering with the team member's time to do their work outside of team. [The team] can keep them out of the ER, out of the hospital, if we can handle the situation within the nursing home that's really best for everyone. We have really been a very successful team 
working together in these discharges. Most of our discharges out of the nursing home were very complicated, going home, needing lots of equipment and needing lots of home care and getting all that set up it was quite challenging, but we did it and we got a lot of people home (PACE IDT Member, K4).

The team is treated as a single entity when quality issues are examined, and is engaged through the IDT meeting to problem-solve and identify lessons learned. The instances where individual providers are singled out from the team are at the point of delivery. This puts paraprofessional team members at a disadvantage. Personal care assistants and drivers most frequently see the patients in their home setting and are most often at the point of care delivery in the home. However, these home-based workers do not have primary representation at the IDT meeting and are represented by proxy. Thus, they may be held accountable more frequently for errors when compared to those who actively participate in the IDT meeting.

A team is considered as an entity unless it's a delivery of a care issue. So it's very different if the team makes a wrong decision about authorizing a particular service versus a mistake in medication administration (PACE Administrator, P4).

Many interviewees felt that subjective issues of satisfaction and creative problem-solving are not fairly represented in the quality measurements. A central 
concept to a participant-focused program, such as PACE, is having the IDT based in a location that can respond and arrange care quickly. The efficiency of team function is an essential piece of maintaining independence for the individual.

I think a successful team is efficient. If a family needs something, can we get it to them in a timely manner? If we have someone on end-of-life and their family needs home care, being able to problem solve and get that started that day or the next day. Doing things efficiently and timely and being able to communicate with each other. I think communication is probably the biggest part (PACE IDT Member, Q5).

Even standardized quality measures can lose meaning when participant context is not considered. Falls were frequently mentioned as an example of how ineffective the standardized measures are for a PACE program. The definitions need to be imbued with the PACE context to be meaningful and accurate as a measure.

Some [regulators] interpret a fall as if they fall in the center, some interpreted that as if they fell only everywhere in the world, some interpreted that as anywhere but a hospital or a nursing home. Some said, "Well I helped her to the floor, that's not a fall." Some said, "Well nobody saw her go to the floor, so it's not a fall." So there were so many different venues. The definitions themselves have to be strong. So if you don't have that consistency that's where the standardization in my mind is 
really important. So define exactly what we're doing is the same (PACE Administrator, F6).

Falls further exemplify that standardized measures not only lose meaning in terms of the definition, but that quantification also loses meaning when considered separately from participant context. One participant may fall a lot while another falls only once a year; however, the consequences of the frequent faller may be less than the participant who falls once a year.

The team really gets frustrated if we talk about the idea of falls. There are people who fall daily. You get to a point where that falling is not a problem. What you're looking at then is how is their quality of life where they're living and how can we protect them to the best of our ability? It moves the focus to how many falls did they have and where are they are, what are the impacts of that fall and that person's well-being (PACE IDT Member, CC5)?

An administrator highlighted the uniqueness of the PACE program, emphasizing that the nature of the people enrolled and the goals of the program do not lend themselves to the standard Medicare/Medicaid/nursing home metrics:

[Quality is] more about the outcomes as opposed to just the numbers. It's just a challenge for [PACE], because you can't compare it because the [PACE participant] is a Medicare person, because they're not. You can't compare us to standard nursing home participants, because they're not. It 
is quite a challenge, we really have to depend on PACE program coming up with some of these areas to focus on and findings that we believe we can really impact. It doesn't matter whether you did admit somebody, it matters what became of that? Were you able to get them happier? Were they able to change their outlook or even though they were sad, did they continue to live and thrive in the community? I think it has to be a little bit deeper than just a count (PACE Administrator, H7).

Some satisfaction was expressed with the current quality measures. Even if the metrics are not specific to PACE and participant context, they serve as a larger indicator of a team that is not functioning well enough to serve the participant adequately. The current quality data collected was identified as useful at the administrative level, but less so at the team level.

I think everything's captured in the quality measurements because if they team isn't functioning well, if they're not communicating, if they're not discussing the issues that need to be discussed, you're gonna miss things and somebody's gonna have a hospitalization, or they're gonna have a reevent, or they're gonna have a fall or they're gonna miss the pneumovac. If the team isn't all there together, you're gonna miss something (PACE Administrator, S2).

Overall, the ways in which PACE IDTs serve the participant and family are largely unrecognized by regulators. Interviewees would like to see several 
dedicated measures that reflect more appropriate representation of patient and family satisfaction and attempts to address a specific participant need through physical improvements to day centers and homes. The concepts of harm reduction, prevention, and comfort were repeatedly reported as under-valued. Interviewees emphasized the realities of working with the PACE population, in particular that the participant may not "get better" and the need to emphasize comfort and independence and the efforts required to make that happen; these can get overlooked by use of the current quality measures.

We have to measure our success by family, caregiver, and participant feedback. Seeing them maintain a placement for an extended period of time. Dying peacefully, for some of them. Getting the acceptance of the family that they are in the dying process, sometimes. We have all sorts of successes and we talk about it and it's brought up every day in our meetings, what was a success yesterday or today (PACE IDT Member, $\mathrm{U} 2)$ ?

Policy-regulative elements provide one way of understanding how IDTs function, with some regulatory actions providing support, and others creating burdens that consume limited time and resources. The three categories that emerged from the interviews were actions of regulatory bodies, issues of resource allocation, and quality measurement. Table 4.1 summarizes the findings for the categories of supports and examples that work within the PACE context. 
Table 4.1: Policy-regulative element categories of support

\begin{tabular}{|c|c|}
\hline $\begin{array}{l}\text { Emerging } \\
\text { categories of } \\
\text { support }\end{array}$ & \\
\hline $\begin{array}{l}\text { Regulatory } \\
\text { bodies }\end{array}$ & $\begin{array}{l}\text { - CMS supports scaling up of PACE, but disallows regulatory flexibility } \\
\text { needed to do it. } \\
\text { - State offices also support scaling up, but offer little regulatory } \\
\text { streamlining to encourage it. } \\
\text { - Disproportionate resources allocated to regulating PACE program for } \\
\text { its size and budget. } \\
\text { - Regulatory approach has changed from improvement and } \\
\text { - } \text { sustainability focused to purposes that appear more punitive in nature. } \\
\text { - } \text { workforce issues. } \\
\text { - Regulation seemingly based on medicalized, acute care models of } \\
\text { measurement, which do not fit with PACE services. } \\
\text { - Redundancy in reporting reflects little coordination on the part of } \\
\text { regulators. }\end{array}$ \\
\hline $\begin{array}{l}\text { Resource } \\
\text { allocation }\end{array}$ & $\begin{array}{l}\text { - Rigid organizational policies provide a framework for decision-making } \\
\text { that alleviates pressure on the IDT in a denial of service to a } \\
\text { participant. } \\
\text { - IDTs require guidance on determining participant needs and wants. } \\
\text { - Grievance process allows for a participant or family to contest a } \\
\text { decision made by the IDT. } \\
\text { - Financial decision-making is ideally contained to the administrative } \\
\text { level and the IDT focuses on participant care. } \\
\text { - Frequent topics of disagreement for IDTs in how to approach the } \\
\text { issues of motorized wheelchairs, dentures, and alternative medicine. } \\
\text { - The valued-added of the PACE program is not adequately captured by } \\
\text { current data sets. }\end{array}$ \\
\hline $\begin{array}{l}\text { The meaning } \\
\text { of quality in a } \\
\text { team }\end{array}$ & $\begin{array}{l}\text { - Metrics provide data, but little actionable information. } \\
\text { - Quality of life not adequately captured or a part of acute care focused } \\
\text { - } \text { metrics. } \\
\text { - } \text { services. } \\
\text { - Efficiency of service provision not adequately recognized. } \\
\text { - Standardized measures lack context to adequately reflect quality. } \\
\text { - Administrators occasionally felt that quality indicators can provide a } \\
\text { - } \text { signal if there are issues with team functioning. } \\
\text { - Metrics lose meaning due to a lack of contextualization. }\end{array}$ \\
\hline
\end{tabular}

\section{Cultural-Cognitive Elements}

Cultural-cognitive elements of institutions are shared conceptions that constitute the nature and social reality of individuals and the frames through which meaning is 
made. The cognitive elements of human existence are of paramount importance, where it is necessary to mediate between the external environment and the response of an individual to that environment which then creates symbolic representations (D'Andrade, 1984). The meanings of these symbols arise through their interaction and transformation as they are used to make sense of what is going on. To create understanding of a situation, one must take into account the empirical conditions as well as the individual's subjective interpretation of those conditions. People in the same situation can perceive that situation very differently. Institutions may impose a particular cultural frame that administrators and providers adopt in decision-making (DiMaggio \& Powell, 1983). Within PACE programs, interviewees discussed cultural categories that support the IDT model of care; some interviewees provided examples of how these categories can also provide challenges to the IDT model. The cultural categories emphasized by interviewees include interdependence, demographics of the participant population, and organizational structure.

Interdependence. "The biggest examples of success that I can think of with regard to the team is when people not only do their jobs but have enough of an awareness and understanding of other people's jobs and roles" (PACE Administrator, B4). The most frequent response when considering issues of culture and team supports was an emphasis on interdependence. Within an IDT model of care, interdependence leads providers to address patient concerns from a point of view of "who on the team is most capable and available?" instead of "whose job is it?" Interdependence at the IDT level requires timely communication among IDT members, knowing their different roles, and access to topic 
experts that can facilitate "proactive preventive care." Interview responses made it clear that interdependence should be improved at all levels: among the IDT, organization, and participant and family.

The social worker will come up to me and say, "Hey did you know" so and so "has an appointment? I didn't know we were contracted with them.” Then I will kind of follow-up and then discuss with the primary care, "Do you want them to see this consult or do we want to go to someone that is in network". It's like proactive preventative care in a cost effective manner is what we try to do. We try to keep them out of the hospitals and try to keep everything cost effective (PACE IDT Member, K4).

Many PACE programs are experiencing growing pains as their enrollments increase and become more demographically diverse. These realities challenge IDT members to understand the multiple cultures and the unique needs of participants. PACE programs reflect the populations they serve, and a change in the culture of the community necessitates a change in the culture of the program.

We've always had a lot of diversity when it comes to culture. We've always tried to accommodate special interests or honor religious beliefs. The company has certainly grown participant wise and we have 
experienced some growing pains. We're still trying to figure things out as they arise (PACE IDT Member, W5).

Interviewees reported a change in the attitude of the participants of PACE programs over the last ten years. IDTs have the complex task of providing the needed care, but also keeping the participant and family happy.

In the beginning participants were dedicated to us, we were dedicated to them. Now we have people who have this mentality of 'well if you're not to give me what I want I am going to leave.' Now the team is not only doing what they think is the right thing, but also they have this compounding factor of how do I please them and not cause our enrollment to go down, to then not cause the administration team to be frustrated. It's quite complex but it's just different. When I think about the type of grievances we got back in the old days were so different from what we get now. They are just from a different culture (PACE Administrator, F6).

Several interviewees said that PACE could learn a lot from hospice. Hospice has a long history of team-based care for the terminally ill. Even though PACE participants are not necessarily in a terminal state, they are towards the end of the life spectrum in years. Their care priorities are about independence and comfort over curative measures.

So the philosophy when I'm doing in-services on PACE and I'm talking to a hospice group I say it's just like hospice on steroids. That it takes the 
same concepts of hospice philosophy of care and expands it out to a longer average length is about 3 years. And instead of end of life focus, our focus is really chronic disease and a wellness focus. It really actually mirrors pretty nicely with the hospice structure and that was one of the challenges we had and it was also one of the benefits we had culturally (PACE Administrator, V7).

Some interviewees linked PACE IDT to the concept of a village. It is important for the various perspectives and disciplines to not only work together, but also to understand what each discipline contributes to the well-being of the frail elderly.

I think it takes a village to take care of an old person, just like kids. It takes a village to raise children because children need all of those voices and perspectives. I think it takes a village to manage a frail, old person because it takes so much work and you need so many different people so you don't burn out. I think you need so many perspectives on taking care of a really frail person and so even though we talk about it being geriatric, I don't know that it's specifically age-based. I think it's definitely frailty based, and I think there's increasing interest in the use frailty as a demarcation, as a syndrome marker, so I don't know that every 75 year old needs a team of people, probably more 75 year olds than currently have teams, need a team of people. I think the frail definitely need a team (PACE IDT Member, M2). 
The interdependence includes the family, who are at the center of the PACE IDT, along with the participant. Many families are accustomed to the standard medical model of care and have had a hard time understanding the value of the IDT.

It's families realizing "I need help" or "my family member needs something to do the day." Getting them to a day center, then realizing "wow, it is nice to have an extra set of eyes on mom during the day" or dad or whoever and it kind of facilitates them to say "what are the next levels of options?" Cause most people going through the aging process don't know what all the layers [of the continuum of care] that there are (PACE IDT Member, U2).

Even though many IDTs acknowledged the value of an interdisciplinary approach, the reality of the working environment can still be challenging outside of the IDT meeting. In practice, interdependence of disciplines may be significantly limited if not all disciplines feel supported in the same way.

The team front that is presented, I sort of feel like you've got to kind of cover your own ass. My experience has been everyone is sort of out for themselves. Now having said that, there are people in other departments that I am very close too and I would do anything to help those other people. I can't necessarily say I would do that for all the departments, but 
I do know that for those departments I feel that have supported me I am very willing and able to support them in return (PACE IDT Member, W5).

Interdependence is also relevant to preventing burnout and facilitating team members to help each other. Finding small accomplishments to celebrate helps keep the contributions of the disciplines at the forefront, and reminds all IDT members of the value of working together.

If you want to talk about burnout, for day center managers in particular, because if you believe in this mission, which they do, then you are in constant battle to try to fight for what you believe is the better things for the person. At the same time you are also fighting against what their right is to make a decision and against that you are also fighting for protection from those people that want them placed. At the same time you are fighting, there is such a conflict there that I think it's such a challenge for those teams to find that happy balance. The teams now you kind of just hold onto the knowledge that are real positive and let them grow, instead of waiting for an entire fantastic story with a great ending. You just kind of find your way by saying today we did three things and they were fantastic (PACE Administrator, F6).

An interdependent team will be more likely to thrive in a PACE program than one that is trying to function independently amongst disciplines. The intensity of teamwork, complexity of patients, and inclusivity of services often 
discourage those who cannot or will not adapt to the model. Several interviewees noted that staff who do not value the team-based approach would only work with the program for three weeks to six months.

When new people come in, they don't realize how lost they are, it's a pretty complex. So, you really are relying on everybody else to kind of help you find your way through and that's where I think the trust building comes in and especially with the team now, we've had some consistency for about 6 months. . If we do have a person who is disrespectful or does not understand the group process or how things work, they haven't lasted very long, it's been pretty consistent that those individuals, cause we have seen them cycle through, last about 3 weeks. It's almost like the environment doesn't support that and so they just don't stay around (PACE IDT Member, R7).

Demographic characteristics. The demographic requirements to receive care by a PACE IDT ensure that the individual is over age 55, requiring nursing home level care and most likely qualifying for Medicaid. As mentioned earlier, each PACE program has a different cultural context responding to the community they serve. Even with these three characteristics in common, variation within these and other demographics is reflected in the organizational culture.

Our client population has become more diverse and the spike in acuity our members is great and varies from so many of those members who are in 
comparison so stable, but we have very consistent contact standards for all of them and we're tasked though with having to spend more time and energy into those higher acuity cases. I know one proposal would be to figure out a way to change that contact standard so it's not as burdensome. Right now for social work and nursing case management it's once a month contact with someone whether they're very high acuity or really stable and lots of support. It is difficult to balance that out when the population years ago used to be much more stable. A higher acuity patient year ago wasn't what it is today (PACE Administrator, B4).

IDTs are challenged in balancing the medical model with communitybased care. Participant behavior and compliance with medication and therapy schedules cannot be guaranteed when the patient is at home. The independence of the individual creates confounding factors to evidence-based interventions, and to some extent PACE IDTs can often assist in home compliance, but not always.

One of those things that surprised us and I think it's kind of a surprise across the nation is the behavioral health needs of the PACE population. In a model of PACE, even though it's interdisciplinary team, it really is a medical model and in the community health world, the psychiatric components of care and the family dynamics, that is one of the bigger challenges of implementing a PACE program in the community, not medical management. You can find doctors to manage the medical component easily, but when you add in independence and they're gonna 
make their own decisions and they don't want to follow the plan of care. Well in a nursing home, you can kind of control that; in the community you cannot (PACE Administrator, V7).

Serving a defined age group of adults 55 years and older does not simplify the model of care. Age differences have many implications for the IDT. The younger PACE participants (ages 55-64) could be with the program for a very long time, and many require mental health rather than medical interventions. Several interviewees anecdotally noticed that the population is getting younger and expressed concern about how this will affect the IDT model.

As we have a different type of person being enrolled in PACE. We're still serving an older population, but we're also being called upon to serve a younger disabled population of 55 to 64 with huge increase in mental health referrals. The mix of people that we need to serve that group is different than what you would've had with the frail older adult that you're getting involved. I see that there needs to be more flexibility versus less, so has it been limiting. I think it's limited, especially in rural settings when you can't find those individuals. When you look at erosion of your reimbursement rates and your costs are going up, all of those things have cost associated with it, so it becomes a very expensive model to maintain. If we can give up and be a little more flexible in our use of that a little more thoughtful, would that then lead to a more sustainable program in the future (PACE Administrator, T2). 
The role of Medicaid in funding PACE along with Medicare provides PACE organizations with a population that is of varying proximity to the poverty line, as defined by each state. Socioeconomic status plays a big role in determining the use of preventive services (Conrad, 2005). By the time many individuals enroll in PACE, neglected medical conditions may have compounded into more complicated health issues. The complexity of these cases requires an IDT to address the range of medical and social interventions required.

Participants generally are low socioeconomic status, but people with all different histories and stories and culture that they bring to our company, our agency, and we feel that's certainly a strength of what we are able to understand about the human condition and how best to serve people. We of course value things like respect and integrity. We place a lot of importance obviously on the quality of our care and again cost effectiveness. Effectiveness trying to be innovative where we need to be and getting people to be expanding on their creativity to do as much together I think and across disciplines to impact members (PACE administrator, B4).

As PACE organizations grow they reflect the communities where they are located more so than the other PACE day centers within the same parent organization. Physical distance between PACE day centers creates a disconnect between the administration and the various locations where IDTs are housed. 
I do see that we have shifted and it's harder now to have that connectivity between the sites because of the size. In some ways many of the sites have to function a little more independently because you don't have that sort of overlap or connectivity the same that I think we felt when we were much smaller. I have always said that the sites reflect the neighborhood that they are in (PACE Administrator, I2).

Working with the frail, sick, and poor elderly requires teams to be comfortable with a certain amount of risk and a general lack of control over a participant's behavior and outcomes. The goal of PACE is not to "fix" the issues but to develop solutions to keep the participants in their homes and not indefinitely confined to a hospital or nursing home bed waiting for a "recovery" that is unlikely to come.

I think in any managed care organization or any time you're working with people out in the community in diverse settings, diverse populations, you have to be risk averse. You have to be comfortable with a certain level of harm reduction versus trying to force this member to be adherent to what I want them to be adherent too? How do I listen to this member's outcomes for their life and figure out how what they're doing planning to what they want for the outcomes for their life (PACE IDT Member, L4)?

Participants of various ages, races, ethnicities, and family support all have different requirements that the IDT responds to in determining the best outcomes 
for the individual. The hope for many administrators is that the culture of respect of a participant's needs will spill over to the IDT and create a culture of respect for each member and what they can contribute to the participant's care.

Finding a way to meet [the participant] where they are in their moments. It's not about us bringing them in and changing them, but rather us adapting to whatever they bring to us. So I think that's really very important. Then of course, we value revering them, integrity, being responsible with resources, and being connected with the community as a whole. All of those types of things are something that our organization strives for not only for our participants, but also then to also staff members amongst each other and then as supervisors to staff, all of those things are just as equally important (PACE Administrator, F6).

Organizational structure. The ways in which PACE programs and their parent organizations are structured have major influences on the operation of the IDT. The organization creates a cultural context that interacts with the PACE IDT in a proactive or reactive manner. The prescribed structure of PACE and its team stems directly from the original On Lok program (Lynch, Hernandez, \& Estes, 2008). As observed with regulatory bodies, there is flexibility and rigidity of the model in adaptation; some concepts translate well to multiple settings, while some do not accommodate as well.

We don't really know what the value added is of all of these services, all of the care management. The case model of care management is a thick 
model of care management. You have nurses, social workers, doctors, NP, PT/OTs, rec therapists, dieticians. You have a very large team and there are certain requirements that they do and we know [PACE participants] have to be reassessed every 6 months, the reality is that we really don't know what the cost, the actually economic value added both in terms of cost and quality to having a dietician assessment every 6 months for example. We don't know. I think in particular because of the kind of cultural inheritance that we have the inherent On Lok model, On Lok operations, or the ideas that formed the On Lok operation. We are always at a cross-roads, but I think we're coming up at a pretty big intersection here in the next 3-4 years with PACE ...Especially the kind of the operating culture where PACE programs have sort of been built from this blueprint (PACE Administrator, C4).

Ultimately, social norms and values that are embodied in organizational practices are constantly challenged by organizational variation. Each program incorporates elements that were legitimated externally and employs the same external criteria to define value. Meyer and Rowan (1991) suggest that this type of isomorphism will create greater stability by providing social definitions that can buffer the organization from complete failure. Even with the external influence of isomorphism, work patterns and processes vary greatly.

You're working out of the same program, you're in the same organization, but there are unique differences because while it's a federal program, it's 
defined at the state level. If there are 39 states involved in PACE, there are 39 different types of PACE programs, so you can't underestimate the state variation and so I've seen that really clearly (PACE Administrator, T2).

Each PACE IDT responds to its own environment. "All of our teams work differently together; we evaluate each of those situations differently, and ultimately they have different outcomes" (PACE IDT Member, K4). Ideally, IDTs are autonomous in their decision-making, but the administration plays a critical part in forming the rational foundations on which those decisions are made.

We are in a constant state of changing, evolving, and adapting. I think that ability for somebody not to be dictated to and/or driven in a certain direction, but rather we look at the need and then make a decision. When I say 'we' I mean obviously administration is what's driving most of these things, but it's not without the input of the teams. I think that gives us a little bit more freedom and opportunity to be who we want to be and not be underneath a grant or mission statement or having to all of us just having an understanding of PACE, but doesn't know how to live it. Having them dictate how to do something, I would think is more a greater challenge than to have the people who are actually doing it to determine how they're going to move forward. I think it's a positive thing myself (PACE Administrator, F6). 
Table 4.2 summarizes the findings for the categories of supports and examples that work within the PACE context. 
Table 4.2: Cultural-cognitive categories of supports

\begin{tabular}{|c|c|}
\hline $\begin{array}{l}\text { Emerging categories } \\
\text { of support }\end{array}$ & Mechanisms \\
\hline Interdependence & $\begin{array}{l}\text { - Legitimization of the team model is made possible when all IDT } \\
\text { members are valued and their roles understood by all IDT members. } \\
\text { - IDTs must respond to the needs of the community and the } \\
\text { community must understand the capacity of the PACE program. } \\
\text { - Improving knowledge of the success of hospice and growing } \\
\text { palliative care models of teamwork may help bridge gaps in } \\
\text { knowledge for acute care settings looking to adapt a more } \\
\text { collaborative approach. } \\
\text { - Interdependence of the IDT is likely to prevent burnout, conflict, and } \\
\text { dissatisfaction within the IDT and with the participant and family. }\end{array}$ \\
\hline $\begin{array}{l}\text { Demographic } \\
\text { characteristics }\end{array}$ & $\begin{array}{l}\text { - Contact standards do not change, even if the acuity of the participant } \\
\text { condition is very low. } \\
\text { - Younger participants (55-64) are experiencing mental health issues, } \\
\text { which is out of the expertise of some IDTs. } \\
\text { - PACE populations are predominantly Medicaid eligible, creating } \\
\text { greater complexity of case management. } \\
\text { - IDTs can gain greater connectivity to the community they are in } \\
\text { compared to the parent organization. } \\
\text { - The IDT members must accept a certain level of risk and that } \\
\text { fostering independence empowers an individual to make poor } \\
\text { choices and well as good ones. }\end{array}$ \\
\hline $\begin{array}{l}\text { Organizational } \\
\text { structure }\end{array}$ & $\begin{array}{l}\text { - By replicating the On Lok model in a variety of contexts, PACE } \\
\text { organizations and, by extension, their teams create a level of } \\
\text { stability. } \\
\text { - The "cultural inheritance" of On Lok may not be sustainable in all } \\
\text { markets. } \\
\text { - State influence through variation in Medicaid policies and } \\
\text { mechanisms cannot be underplayed in determining the sustainability } \\
\text { of PACE. } \\
\text { - Rigid policy structure and rules provide needed structure and } \\
\text { support for administrators, but administrators need more flexibility } \\
\text { in tailoring PACE services to the communities they serve. }\end{array}$ \\
\hline
\end{tabular}

\section{Normative Elements}

At the core of the normative element of institutions is the emphasis on normative rules. Normative rules introduce prescriptive, evaluative, and obligatory dimensions into social life. There are two components to a normative institution: values and norms. 
Values are conceptions of a preference together with the construction of standards, which provide the basis for evaluation (Scott, 2008a). Norms specify how things should be done. Some values and norms are applicable to all members of a group, whereas others apply only to selected types of actors or positions. Normative elements are typically seen as acting as constraints on behaviors, but they also allow for social action. They confer rights and responsibilities, privileges and duties, licenses and mandates (Scott, 2008a). The underlying concept remains the focus on social obligation for performance. Interviewees highlighted organizational mission and values, leadership, and professional boundaries as the primary categories of support within the normative element.

Organizational values and mission. The PACE model is based on the belief that it is better for the well-being of older adults with chronic care needs and their families to be served in the community whenever possible. PACE participants meet the following criteria: individuals age 55 or older, certified to need nursing home care, are able to live safely in the community at the time of enrollment, and live in a PACE catchment area (National PACE Association, 2012). "We promote being member-centered, looking at cost effectiveness, promoting team work, those kind of pieces add to the value on a daily basis and we are aware of that" (PACE IDT Member, L4).

Our organization is there to help the seniors to be as independent as they are able with our assistance and to live in their home in their communities; to stay there rather than - I believe when this all started it was trying to get people out of the nursing homes and staying in their homes longer, which is great (PACE IDT Member, AA4). 
Interviewees consistently stated that the PACE model is not a medical model; it is a dynamic way of decision-making and there are few "fixes" available to IDTs to treat participant issues. This may run counter to the norms of many parent organizations that are primarily acute care providers. Many providers who are accustomed to working in acute care settings find it difficult to change practice style and work in an interdisciplinary, participant-centered way.

There is a big difference, I think; one of the keys is who the providers are. Providers are looking for state of the art interventions. That is not necessarily the PACE model, because we are not - particularly if they come from a fee-for-service environment. We are not a fix-it group that is not our role. So often times the information we get from providers is not vetted for that's expensive and equally effective alternatives. They are not vetted for its relationship to the mission (PACE Administrator, A3).

A central tenet of PACE is to keep frail elders out of hospitals and nursing homes, living independently in their communities for as long as possible. The IDT must be vigilant in reminding its members of the goals of the program.

Whenever we have our meetings or whenever we discuss participants, we see that focus of how we can get them independent. What can we do as a team to support that? I have seen that very much. I'm really happy for that because I joined for that purpose (PACE IDT Member, O3). 
In order to orient new staff members to the unique mission of PACE, the mission and values statements are given a prominent place in trainings, meetings, and collaborations.

It's part of new hires orientation every single employee gets an orientation to mission when they first start. It's reiterated in their orientations at the site level, we have it posted in different places in terms of what the mission and core values are, and then people really bring it into the discussions. Our core values include respect, for example, so it's not uncommon when somebody's having a conversation or if there's something that's an issue that's coming up or even in the context of leadership team decision. You might call upon how does that relate to our values, and so there's a true attempt at values integration at every level within the organization, so I think it's really very real and felt that way (PACE Administrator, T2).

Interviewees rarely spoke of improving the medical condition of PACE participants. The focus was more social than medical, and medical needs are often weighed against social needs. "The IDT must move towards giving the best possible care with the mission in mind; also to stay in business and for the IDT to provide a quality of life that allows them to participate in society" (PACE Administrator, A3). By virtue of having such an extensive IDT, the all-inclusive model requires more non-medical decision-making in many cases. IDTs value the 
all-inclusive services to their participants, because each service integrates many social components that contribute to the well-being of participants.

Essentially, we try to work together and strive to keep at-risk individuals living at home and keep them out of ALS and nursing homes. That's not always the case, but that's what we try to do through partial day services, transportation services, and home care services (PACE IDT Member, W5).

The PACE program not only provides comprehensive care, but it is patient-centered. Long-term care has been at the center of the culture change movement, which seeks to put the patient at the center and give them a voice in their care. Every interviewee made a reference to "The common belief and value, held by all IDT members, is to do all you can for the participant" (PACE IDT Member, O3).

We are in a constant environment of change and one of the first things I learned about $[\mathrm{PACE}]$ and it has stayed true to this day. We are constantly having to change ourselves and be flexible and try to be innovative with how we do things, how we try to support the members in our program...I think for the most part they really embody the larger agency values; certainly member centered. That's in contrast to some of medical models that get inserted a lot of times; keeping the team aware of member autonomy and self-determination to live their lives. I think we want to see 
positive health and safety outcomes for our members. We want all of our members to have a positive quality of life as much as they can. I think the people on the teams and the people at the site here and really throughout all really take pride in their job and are very interested in seeing members that our happy and healthy (PACE Administrator, B4).

Administrators and some providers also emphasized the financial risk involved in a program like PACE. Even if they want to do "everything they can for the participant" there is a very real limit financially. "Our goal is to optimize their quality of life and optimize their ability to maintain in the community, but yet keep our resources utilization effective and to meet their outcomes, to try to and stay - not go up and beyond, offer services that they don't need" (PACE IDT Member, K4). With interest increasing in expanding and scaling the PACE model, the IDT decisions for care must always include a consideration of cost and affordability, not only for the participants but also for the organization.

Our organization's mission and values is really to provide - if I summed it up in a nutshell, it's to provide effective and cost effective care to the elderly. That mission drives everything that we do from a team level on up. We expect all of our team members to know what our core values are to a certain extent, not to be able to recite it verbatim. They should have a gist of the core values of the agency and practice keeping those core values and the mission in mind (PACE IDT Member, L4). 
PACE is an intensely mission-driven program and this places a complex burden on IDTs to not only make the best care choices possible for the participant, but to also make the best choices for the organization.

Leadership. "It's interesting how leadership makes itself known" (PACE Administrator, H7). Formal leadership structures that supervise the IDT members do not mirror the collaborative nature of the IDT. Each health profession discipline has its own hierarchy outside of the team. The integration of leadership within and across these disciplines is highly variable and, in some organizations, the collaboration within an IDT is contrary to the approach of much of the rest of the organization.

We have a supervisor, like a social work supervisor and transportation supervisor and they have leadership within their own little department, and I think some of those people are considered informal leaders for sure. But they're on paper, they're responsible for their department. So I think I'd say the formal recognized leaders would be the director, the center managers, the nurse practitioner, I guess that might be it (PACE Administrator, J1).

Even with the complex leadership structures of the PACE organization, informal leadership has a significant place within each discipline, and varies according to the participant. Many of the formal leaders bridge the disciplines and informal leaders act as bridges from the participant to the IDT. For example, one administrator said: 
I wouldn't say it's a discipline [that formulates the leadership role], but I'd say it might be one person within that discipline. You see that in any team dynamic. There's going to be those folks that just kind of when something needs to get done, they just kind of charge forward and do it and people will tend to follow because they've got that conviction around what they're doing. I do think we have many informal leaders in our organization (PACE Administrator, E5).

As discussed in Chapter Two, a team physician may be granted greater clinical authority than non-physician team members. Within a structure such as the PACE model many decisions are not clinical in nature, creating opportunities for other professions to act as leaders on team-related decisions. Given this, the physicians are still provided with the recognized authority and the onus is on them to defer and delegate that authority, especially on non-medical issues. For example and administrator said,

A physician can be very powerful. If a physician pooh-poohs something, it's over. So early on, you really have to engage the physician to make sure that they understand how powerful they can be because I think [our physician] has absolutely embraced the interdisciplinary care model and really looks to her team before she needs to get involved (PACE Administrator, BB2). 
Frequently interviewees projected that to engage a physician in the team model takes about one year from the day they are hired by PACE. One goal of the organizational leadership structure of PACE programs is to develop the physician as a culture change agent to help in legitimizing other members of the IDT.

There is not a formal hierarchy of who trumps what, but ultimately, the medical director is the one who are managing the medical needs of this patient. I think ultimately, the physician will say this is what we need to do and writes the order because in health care, a nurse can't write an order and make something happen. Recommendations can be made, I think that's why you have to have the success of PACE is relies on having a very involved physician (PACE Administrator, V7).

Health care workforce licensing, scope of practice and long established hierarchies can cause team members to struggle with finding their voice within the IDT. This is observed in the integration of paraprofessionals and entry-level staff into the IDT.

But as far as the PCA (Personal Care Assistants) or the care aides, they attend the meetings and I, again, having come from an organization where it's not this way, I'm just astounded at how they have an equal voice and they're not afraid to speak up. So we may have a doctor saying, "I want this person to be brought in every 3 days a week," and the aide may say, 
"But doctor, at one o' clock every day they're screaming they want to go home." And the doctor will say, "Oh, okay. Well then let's bring them in twice a week." So they really do have the same voice in those IDT meetings (PACE Administrator, S2).

In many cases, the actual leader of the IDT is the facilitator. For each PACE IDT meeting there is a designated facilitator who conducts and manages the flow of the meeting. The facilitator can be anyone, but is most often a supervisor or manager who is not a formal member of the IDT. Some programs rotate the facilitating position, while some do not. When it comes to finding voices for all disciplines and assuring equal participation in an IDT meeting, the facilitator's job can be daunting.

When I am facilitating IDT I try to make an effort to include the lead aide. If they were sitting in the meeting and didn't say anything I will ask her "Hey do you have any input? What have you seen in the day center this week?" Do we know how this person is doing on the bus? Has anybody heard from the family if they've noticed any changes? Even outside of the team what are our community partners' saying about what is going on with the person? As a facilitator, just trying to make sure everybody's voice is heard during a discussion. I think if you work with the team and build a situation where our common practice is that everybody provides input; it builds a culture where everybody's input is valued (PACE Administrator, I2). 
Even though the facilitator is often not a formal member of the IDT, they are critical in developing team communication and culture. The facilitator also acts as the bridge among higher management, external governance bodies, and the IDT.

The facilitator becomes sort of a de facto leader, both in directing the conversation, but also in providing feedback...you know I mentioned before we do have some bottom line policies and guidelines on some things, so providing some of that information so there's transparent leadership that provides the leadership in team (PACE IDT Member, M2).

Professional boundaries. Professional boundaries provide parameters for how and where IDT members can work; this is first defined by state scope of practice regulation and second within the IDT. Each IDT member has a variety of competencies to take on certain roles. Roles within the IDT are based on an individual's competency and may create different roles for IDT members than what can be seen in other care settings. In some cases, even if certain tasks are covered by defined scope of practice, the PACE organization may modify the usual scope of particular professional groups in order to best serve the care needs of the participant.

The thing about a PACE interdisciplinary team, which makes it hard for many people that come from the outside, is everyone's got a voice. I mean it really doesn't matter if you're talking about polio, if the activity assistant wants to say something about it that they think is relevant then 
their voice holds equal weight on the IDT as with the doctor. That's really difficult for certain disciplines to work with and makes it a challenge as a facilitator is making sure that everyone is paying attention to what the activities assistant has to say (PACE Administrator, D5).

Boundary issues also arise when different interpretations of participant safety become known. These disagreements are boundary issues when the IDT struggles to define who has the authority to define what is safe for a particular participant.

Early on in PACE to try to make a decision when everybody has input into it, the challenge is who has the final say on what direction you're going. So, the easy decisions, no, it's very easy, people can all agree, and it can be kumbaya. The challenge part is who thinks somebody is safe in the home, PT might not think they're safe, but the medical think they're okay and the social worker thinks it's okay and the nurse might have a different perspective. That's when the disciplines' boundary issues become more of a challenge (PACE Administrator, V7).

Many non-physician members of the IDT must learn the scope of practice boundaries that apply to their teammates. In clinical settings such as a PACE day center, informal advice cannot be given to a participant by an inappropriately credentialed staff member. Even seemingly simple requests may need to be passed to another IDT member who can appropriately respond. 
We have a day center here on campus that a lot of folks come in to and the participants will trap social work as we're walking through and say "I have a head ache, what should I take for it?" We have to say, "You need to talk to the nurse" or "You need to talk to the doctor." We can't tell them to take Tylenol because that's outside of our scope of practice. If you're not used to working in the medical field and you're just kind of used to saying "oh, just take a Tylenol". So we're very cautious of that, that doesn't happen very often cause our health aides can't pass meds the way the license works here. They can only say, "Have you taken your meds today?" But they can't actually pour the meds out and put them on the counter because that's considered administration (PACE IDT Member, R7).

However, some physicians express uncertainty about their role. The uncertainty stems from the fact that the nature of care required by many PACE participants does not require medical oversight. "I find it challenging when much of the work is getting done by other people that I start wondering what am I doing here? What is my role? And actually, that's been challenging" (PACE IDT Member, M2).

Normative elements support IDTs through organizational mission and values, leadership, and professional boundaries. The organizational mission and values must support the collaborative IDT model. Leadership allows the IDT to act as the primary decision-maker and intervenes only if necessary. Professional 
boundaries are specific to the IDT composition along with scope of practice

regulation. Table 4.3 summarizes the categories highlighted by interviewees that

support IDTs and their mechanisms.

Table 4.3: Normative categories of supports

\begin{tabular}{|c|c|}
\hline $\begin{array}{l}\text { Emerging } \\
\text { categories of } \\
\text { support }\end{array}$ & \\
\hline $\begin{array}{l}\text { Organizational } \\
\text { mission and } \\
\text { values }\end{array}$ & $\begin{array}{l}\text { - PACE IDTs are driven in their decision-making because they offer } \\
\text { participant-centered care. } \\
\text { - IDTs provide all-inclusive care that rarely is promising any "fixes". } \\
\text { - Maintaining elder independence is central to IDT decision-making. } \\
\text { - IDTs constantly try to minimize or eliminate time spent in hospital or } \\
\text { nursing home. } \\
\text { - The IDT meeting is the forum for revisiting the mission and focus of the } \\
\text { PACE program. } \\
\text { - Social needs are just as important in care decisions as health needs. } \\
\text { - Participant self-determination has drawbacks for many practitioners, as } \\
\text { greater participant independence does not necessary lead to higher rates } \\
\text { of compliance with instructions. }\end{array}$ \\
\hline Leadership & $\begin{array}{l}\text { - Leadership is formally constructed by disciplines, which act as the first } \\
\text { level of supervision outside of the IDT. } \\
\text { - Interdisciplinary supervisors oversee multiple disciplines. } \\
\text { - Often informal leaders often act as proxy advocates for participants and } \\
\text { their families. } \\
\text { - Physicians are still imbued with the most authority and decision-making } \\
\text { power. The onus is on the physician to share that power. } \\
\text { - There is little opportunity for team members such as the PCAs and } \\
\text { drivers to take on any leadership roles as they are rarely included in IDT } \\
\text { meetings and are represented by proxy. } \\
\text { - The facilitator is a strong formalized leadership position, responsible for } \\
\text { the IDT meeting flow where most of the IDT communication and } \\
\text { decision-making happens. }\end{array}$ \\
\hline $\begin{array}{l}\text { Professional } \\
\text { boundaries }\end{array}$ & $\begin{array}{l}\text { - Scope of practice boundaries are defined by the state, but PACE } \\
\text { organizations may restrict some privileges in order to meet PACE } \\
\text { practice regulations. } \\
\text { - It can be difficult for team members to work where boundaries are } \\
\text { changing depending on the participant and situation. } \\
\text { - Disciplines often disagree over interpretations of patient safety. } \\
\text { - In order to improve interdependence, IDT members and entry-level staff, } \\
\text { alike must learn the range of competencies and scopes of practice. } \\
\text { - Non-medical professionals must operate within their scopes of practice. }\end{array}$ \\
\hline
\end{tabular}




\section{Relational Elements}

The relational factors of teams are represented in the literature through

discussions focused on socialization, team composition, and team roles, and processes as illustrated in Chapter Two. Professional power is a complex issue within interdisciplinary teams. The adoption and use of interdisciplinary teams can hinge on physicians' willingness to share power (Mackay, 2004). Even if there is a willingness to share power at the surface, the degree to which that power-sharing is evident in practice can be questionable (Gibbon, 1999). Besides identifying who has power, one must also consider the other side of the power relationship and consider how those without power respond. Resistance to power has also been observed to be a detrimental force (Foucault, 1978). Power resistance may be passive, observed as apathy toward teamwork and not “pulling one's weight", or active, as seen in sabotage efforts of other team members or team projects (Delva et al., 2008; Reeves, 2010). In order to avoid the adverse effect of power within a team, relationships require daily management to assure integrity and legitimacy of the team and their work. Team roles and communication within the IDT were the primary categories that internally support IDTs.

Roles. Since many disciplines are monitored by a third party through licensing and scope of practice laws at the state level, role delineation is important to avoid professional boundary infringement (Booth \& Hewison, 2002; Reeves, 2010; Stark et al., 2002). Although roles in health care are normatively defined through third parties, the extent to which PACE IDT members work at the "top of their license" is defined amongst 
the team. Not all IDT members are licensed, and even if they are, as described in the previous section, the actual scope of practice may be adjusted according to organizationally defined team needs.

When I try to visualize what the social workers here do I kind of think of it as like the wheel of a bike where there is the center and then there are different spokes coming out. So let's say the different spokes are like PT, OT, the day center, the nurses, the home care staff, that sort of thing and I kind of visualize the social workers as being like the middle of that wheel, because we're kind of like the glue that holds everything together. We tend to do a lot of the follow-up to make sure that things are done (PACE IDT Member, Q5).

Roles within the IDT are persistently in flux. Interviewees expressed that the role of the physician is most significant in medical decision-making. Each program expressed a desire to have greater regulatory flexibility in integrating a stronger presence for a nurse practitioner (NP) as a primary care provider (PCP) for PACE participants. From a regulatory standpoint, CMS restricts PACE programs from utilizing NPs as PCPs even if they have autonomous practice rights within the state. Only one site in the study reported that they had successfully acquired rights via waiver for the NP to act as an autonomous primary care provider. 
I'm thinking about cases that it's very clear that [physicians] have a role to play and they're probably going to be the head with a highly medicalized sort of intervention. When I think about most of the other discussions that are medical but are interdisciplinary in nature, involving housing issues or some other psychosocial issue that someone is dealing with and they seem to be open. I think a large part of it too has to do with the nurse practitioner piece. I think the nurse practitioner as a partner in primary care a lot of times serves as a bridge is how I've always sort of approached them, between the doctor and the more medicalized side of care and bridging that with the nursing and psychosocial and community based sort of issues that can come up. So I think they do a lot to really help balance things out as well (PACE Administrator, B4).

Frequently PACE IDT members identified advocates who serve as proxies for family at the IDT meetings. These proxies become necessary because the IDT members are either stationed at the day center or are out on home visits, and those that are at the day center may not have any first-hand knowledge of the participants' living conditions.

I feel like my role is to support the families, but also one of the big things that I see, as my role is to do training with the people who I think are the heart and soul of the system. The home care workers go in 2-5 days a week and do showers, get people dressed, and do all kinds of things to support [the participants]. With some training, we will go out and meet 
them in a house that they're having a problem transferring somebody or something like that and provide new techniques and skills to help them do their job (PACE IDT Member, CC5).

It is often up to management to help define the roles of various managerial levels and make resources available to support the IDT. The involvement of administrators with the IDT is highly variable. Many are former practitioners who can advise on some difficult clinical decisions. Providing information to the staff at all levels, and defining the expectations and priorities for practice, is a part of the role many administrators carve out for themselves. Some frequently attend IDT meetings, and some rarely attend. Administrators can find adjusting to the PACE model as difficult as the IDT members.

I see myself very much an educator. My job is to serve those people that work here as much as the participants do. I spend a lot of time in the team reminding people of the regulations and the requirements. IDTs understand what we do and how we do, it isn't just an arbitrary - you know we woke up today and decided to do it this way. I think that sometimes it is hard when you're trying to learn something new and when things change that it's helpful to have somebody reference okay this is why it's changed and this is how it's been. So as far as my role with the team, I' $m$ in the team, I do still go to the team meetings, I see myself more as a consultant (PACE Administrator, G6). 
My role in the IDT, when I first got here, I found to be very challenging because having come from long term care, I was the queen and I made the decisions and I was the person in charge and I always sat at the end of the table and people looked to me for direction. I would start shifting myself down so I wasn't at the head of the table. I found I actually had to sit outside the ring in order for people to not look at me, because as the manager, when they're stuck, they just naturally look at you to help make the decision and send them one-way or the other. I purposely try to remove myself from the inner workings of the IDT so I'm not relied upon (PACE Administrator, S2).

Communication. Personal Care Assistants (PCAs) and drivers are members of the IDT. These team members were not required to attend IDT meetings at any of the sites studied. The supervisor, who would relay messages back and forth, represented most PCAs and drivers at IDT meetings. Reasons were varied as to why PCAs and drivers were not required to attend team meetings with the rest of the IDT. "They are so busy" and "their English is not very good" were two of the reasons provided. The IDT participation by proxy structure minimizes contributions of these members of the IDT. Communications from PCA's and drivers are often of an urgent nature, but input is rare, as reported by interviewees.

We don't do enough communicating with either of those departments [PCAs and drivers] and likewise they don't do enough communicating with us. I know my supervisor who is the site administrator here has 
tasked the transportation supervisor to attend ' $\mathrm{x}$ ' number of meetings every quarter, just to have a presence at the team meeting, share issues that come up or we can share with him and that has always fizzled out. That certainly hasn't been the right way to try to improve communication with those departments. We don't have anything formally in place to facilitate consistent communication with those departments (PACE Administrator, B4).

Well we know the main IDT members have a strong voice. We struggle to make sure we hear the voices of the driver and maybe the supportive home care aid, but the RNs, the social workers, the NPs, the MDs, the dietician we don't struggle with that. They are around the table all the time. Where we struggle at times is to get the voice of those more distant team members (PACE Administrator, P4).

Interviewees perceive conflict as a necessary part of team communication. The benefits of an IDT should be in the synthesis of different perspectives to develop the best solution for the participant. The critical function of the resolution process is to navigate through it and not let it devolve into bickering and alienation of team members.

In one situation, we had one $\mathrm{RN}$ who felt more strongly the meds to be administered to [participant], and then we had the [Social Worker] saying well, I think that's not the reality of who this person is. I love those 
discussions because I feel like everybody's perspective is on the table. The worst agreement is the agreement where not every stakeholder is represented. Short of having the patient present, people can empathize and see it from their perspective, that's very helpful (PACE IDT Member, Z7).

I have always called it therapeutic conflict. I think it's so important for us to have that. That it's really not PACE unless you're coming up to those times where you're touching onto the boundaries of what that person might be able to take, because you're not going to agree with them. It's how you facilitate that and how you support that and how you show them that that is what is expected of a good team. It's not called bickering, that's called finding the best answer for the participant (PACE Administrator, G6).

The IDTs avoid negative conflicts by remaining cognizant of their mission. IDTs keep the focus on the participant and their family for decisionmaking. The heavy workload of IDTs also removes an excess of downtime that has been perceived by PACE staff to breed negative conflicts.

With the people that we have in place on our team right now, we are sure there are times that we might disagree. But our team members don't have that political cattiness, there's no backstabbing, we're a very patientdriven team, there's a lot of passion to care for the individuals and quite 
frankly I don't think there's time for us to get into all that other stuff, we're pretty busy. Our team members just have a lot of leadership and professionalism. I think that translates into the care that we do cause the participants see how we interact with one another and I think on a personal level (PACE IDT Member, R7).

To me, it's gauged around patient care, but then there's still that, for lack of better words, collaboration just by talk of how this participant is and what's gonna help them today? So there is still that level of connection that they have, but they've focused the talking around a participant (PACE Administrator, X2).

The value of an interdependent culture is not lost at the relational element. IDTs have high rates of retention (Mahmood-Yousuf, Munday, King, \& Dale, 2008) and this familiarity allows for a comfort level that encourages all team members to participate actively. Interviewees also reported that active participation creates more respect for all disciplines and what they can contribute to each meeting.

You're just as important as I am, nobody's opinion is valued more than the other is. Nurse's opinion is not valued more than the dietician's opinion or my opinion or the Adult Day Coordinator's opinion. Everybody gets their voice because it takes all of us because everybody lays eyes on them, they look at them in a different view, and I think that's great because you 
put it all together and then you get a more complete picture (PACE IDT Member, Z7).

There are consistent challenges in communication amongst individuals, and even those who work for PACE and participate in IDT meetings can act in ways that are perceived to be detrimental to the discussion.

"You need to talk to the doctor"... [The IDT] wanted me to talk to them about being more respectful in the meeting; they were reading the paper and made some references that were not particularly respectful. They wanted me to talk to them and figure out what's wrong with them (PACE Administrator, BB2).

Creating a voice for one's discipline within the IDT can require constant maintenance. Team compositions are fluid, according to interviewees, and there is a responsibility for each discipline to establish what their contribution within the team will be if they believe it is important. It may take time for the role to gain traction and be an accepted part of the team working process.

I can't tell you how many times over the years I've heard "nobody's gonna do anything about it anyway, so why should I bring it up again? I brought it up; I told the whole clinic, yada, yada, yada." I try to tell them that just because they may feel that way, that sometimes it's about behaviors and not finding a medicine. The doctor doesn't always have a magic bullet to fix it. It doesn't mean nobody's listened to you. When you 
stop bringing things up, you never know when there might be solution that could be thought of, so even though you feel like you've said it over and over, people change, the situations change and it's important that you still bring these things up (PACE Administrator, J1).

Attendance and active participation in the IDT meeting is the key to internal team legitimization of the disciplines present. Discipline-based rifts are still experienced within IDTs. The IDTs can only be as participant-centered and interdisciplinary focused as the individuals on the team.

It seems that if you're not there to speak up and represent your department, it seems like the responsibility is placed on you out of convenience for other departments. I do know that there are some fellow coworkers who are as just as frustrated as I am. I guess I can only really speak for myself, but that's what causes me to be to be defensive in those meetings and I also don't want to say anything unless it's a safety issue or my staff are depending on me to bring it up. Sometimes when I have responded in those meetings, I am made to feel like, I don't want to say an idiot, but essentially that my opinion doesn't matter (PACE IDT Member, W5).

Relational elements support IDTs through roles and communication. Interviewees discussed the complexity of role definition. The roles defined within the PACE organizations are highly flexible and responsive to the particular needs 
of each team and participant. Communication is challenging and ensuring equal time for each member becomes an issue especially with IDT members who do not attend the meetings in person. Disciplines may disagree, but overall interviewees found disagreement and conflict to be a positive part of the process to finding innovative solutions to participant issues. Table 4.4 summarizes the findings for the categories of supports and the mechanisms that are at work within the PACE context. 
Table 4.4: Relational categories of supports

\begin{tabular}{|l|l|}
\hline $\begin{array}{l}\text { Emerging } \\
\text { categories of } \\
\text { support }\end{array}$ & $\begin{array}{l}\text { Role delineation happens through scope of practice law and social } \\
\text { definition by the IDT and organization. }\end{array}$ \\
\hline Roles & $\begin{array}{l}\text { In many teams, there is one person whose role it is to bridge } \\
\text { communication gaps outside of the IDT meeting. }\end{array}$ \\
- Physicians maintain much of the control over decision-making. \\
PACE regulations prevent utilization of NPs as PCPs on an equal \\
footing with physicians. \\
- Even with the emphasis on maintaining independence and keeping \\
participants at home, only a few of the IDT members ever visit the \\
participant's home. \\
- The roles of management and supervisors are highly variable; many \\
interviewees described part of the management role in terms of \\
education and training for IDT members.
\end{tabular}

\section{Procedural Elements}

The procedural factors of teams are represented in the literature, as discussed in Chapter Two, through discussions around recruitment and retention, time and space, and training and education. Meeting the health care needs of the growing aging population through alternative workforce models has been the focus of many studies as discussed in Chapter Two; of particular interest to researchers is the question of how the growing 
aging population will affect the workforce needs for providing care for the elderly (Baxter\& Markle-Reid, 2009; Bragg \& Hansen 2011, Miller, Booth, \& Mor, 2008; IOM, 2008; Stone, 2004). There are persistent recruitment and retention issues in geriatric specialties of most disciplines (Coogle, 2005; Miller, 2008; Stone, 2004; Warshaw, 2003), including medicine, nursing, allied health, and entry level staff (Bragg 2010; Gawande, 2007; IOM 2008; Stone, 2004).

Another important characteristic in thinking about teams is time and space. According to Durkheim (1976), time and space are intertwined within the social organization of work. As health professions are training their students to practice in interdisciplinary teams, and health reforms are restructuring the way care is provided in the U.S., the understanding of the influence of the institutional context will contribute to the sustainability of interdisciplinary team models of care. During the interviews, the three categories of support identified were recruitment/retention, time/space, and education/training.

Recruitment and retention. Organizational hiring practices and provider training affect the ability of teams to collaborate, coordinate and network, all of which play a part in legitimizing and supporting teams at every level of a health care institution (Reeves, 2010). A high quality workforce requires a significant social investment in education and ongoing training of workers. There are three areas of emphasis to consider when addressing the issues of the LTC industry: initial education programs, continuing education, and faculty recruitment and retention (Reeves, 2010). Formal educational institutions and programs should be monitored for quality and competence of graduates. 
Programs need to address the issues unique to long-term care populations: disability, issues with cognitive functioning, and quality of life. Programs must also train the workforce to work with a multitude of other occupations and professions, and to recognize the importance of the unpaid caregivers and the patient as a part of the care team. Students of all health professions should be required to experience LTC placements in the community that complement classroom instruction.

I think probably more importantly there are very few geriatric-trained staff that we can find. So we take them from the community of health care, which can be - listen you would be hard pressed unless you were in a strict pediatric program, you are going to see elderly, because it just is a very large portion of the population. But they are not trained as such. They don't even get that not end of life per se, but that more palliative and comfort care mode, that's not as comfortable for them. It takes a lot of education to get them to come around (PACE Administrator, G6).

PACE regulations stipulate the credentials of team members, and staff must have at least one year of experience of working with the elderly. Given the current shortages in geriatric specialties, finding qualified providers may become a larger issue as the population gets older and PACE programs attempt to grow and expand. "Because we can't seem to find a physician, but I hear that's something that's happening all over" (PACE Administrator, S2). 
Trying to find someone who actually has geriatric experience and a desire and a passion to work with a geriatric population can be challenging. You put on top of that that the social worker specifically within PACE is a pretty eclectic role where we are doing some clinical work, some case management, a lot of work with families and caregivers and moving across levels of care and it's not a role that is really well replicated anywhere else. We also have a requirement that we can only hire Master's level practitioners you are looking for someone with a Master's Degree, preferable an LCSW with at least 1 year of geriatric experience, who wants to do a combination of home health, hospice, case management, and clinical work. It can be a challenge to find someone who meets both the paper criteria, and they are going to be a good fit with the team. We have had people over the years who are great clinicians, they are great workers, they great with the patients, great with the families, but they are not a good team member and that can be challenging (PACE Administrator, I2).

The issues in recruitment are not limited to physicians. Therapists are also becoming more difficult to find. This is an issue for PACE as each team requires three therapists (occupational, physical, and recreational). There is also a difference between the retention rates of professional and entry-level staff according to interviewees. Administrators interviewed reported that retention is very high among professionals in the PACE program. However, PACE is not exempt from issues in retaining entry-level staff. 
In the professional staff [retention] is good, it's pretty low. But in the entry-level staff, unfortunately we still experience significant, $35 \%$ turnover. It's just despicable. I'm having a consultant coming in, we' re going to do some personal care assistant group work, and we're going to see if we can put some suggestion groups together and get their ideas on a better way to do this. Because I think, they just don't feel empowered to make a difference in their job. So we're going to start that (PACE Administrator, G6).

When considering retention issues, administrators are mindful that frontline staff are not integrated into the IDT in the same way as the professional staff. However, there appears to be a stable work environment and schedules for entry-level staff.

I know for the [entry level] staff it's probably primarily pay. They've got a pretty flexible schedule and they've got weekends and holidays off. The staff is - I'm trying to think of a PC way to say it, they don't necessarily have a lot of education. They've probably always had low paying jobs. I make good money and the flexibility that I'm able to come and go if my daughter is sick or if I need to leave in the middle of the day for a doctor's appointment. Again, I've got a young daughter, I value the weekends, and holidays off, that's what I need right now, so in that aspect that works for me (PACE IDT Member, W5). 
In the professional staff, those folks that are probably holding some kind of advanced degree, or whatever, it's good. PCA turnover is higher and it's for reasons that don't make a lot of sense. Why would you leave for 10 cents an hour, that doesn't make sense? If you liked it here, you wouldn't leave or 10 cents an hour. The work is hard and I don't think that the personal care assistants are engaged in the same fashion that the professional staff are. So it's a problem for us, I openly admit that and I hate it (PACE Administrator, F6).

IDT members, and physicians in particular, have difficulty being an employee on more equal footing that they may be accustomed to. Retention hinges on the willingness of individual team members to participate in the IDT and support the IDT model.

Time and space. Dedicated time is perceived as being necessary as a way for teams to develop trust, respect, and interdependence. This is developed not only through formal communications and meetings, but also through informal communication during the day. Talking outside of the formal IDT meeting setting can be perceived as unproductive time, but it is critical to team building.

One of the oddities about this clinic is that it was intended for fewer people and as a clinic, it's not really well laid out and we've always felt like we're on top of each other. I could tell you every personal detail about just about everybody here because you hear everything that goes on. I was 
actually kind of looking forward to a little peace and quiet. The downside of that is I think there used to be a lot of informal communication, unintentional and informal communication that now isn't happening. You always knew what was going on; there were no surprises because you'd hear it. Now, maybe not so much (PACE IDT Member, M2).

Spatial boundaries create barriers to many health services needed by a patient; these boundaries also separate providers who should be co-located according to interviewees. However, a small day center and office space can inhibit co-location of IDT members as other members have to find office space elsewhere. It can also reduce the number of basic facilities a day program would require.

The thing that really jumps out at me is at the smaller site here not all the disciplines are located in this building. Some are at different buildings, like the dietician and behavior health, and the therapy department, all of them come to the day center once a week, but they are not within our building. So we do a lot of e-mailing, which is very different from the past. We come together once a week and then if there were things that happen in between we would e-mail each other. That's a big difference and that's the way of the world right now (PACE IDT Member, AA4).

No interviewees complained of having too much space within the PACE day centers. Even as spaces are dedicated to the program services, PACE 
programs also grow by increasing number of participants and staff. Rarely is there an empty space for which there is not a purpose or use.

It's just a small space. We just have to be a little more open to sharing and really, that's understanding that somebody's not trying to be rude or crowd in on you, but it's that it's a small space and we have to share. You don't need a formal meeting with a table to meet, you can just stand and talk about something with three or four people in a corner of a room and be appropriate. Because the more space you have, the more you fill in with activity and with people and so, it's always a limitation. Flexibility is the big word I can think with the space. It's also very nice, though, to be a small site because it does put some limitations on how many people can come in in a day so that you're not overwhelming the participant in the long run (PACE IDT Member, U2).

The overall response of interviewees was that the relatively small spaces facilitated better IDT functioning and communication, through the informal interaction of all PACE day center staff throughout the day.

Training and education. IDT members require a comprehensive understanding not only of their own role, but also the roles of other colleagues. PACE programs vary widely in how they train and prepare their staff to work in the collaborative teams required by PACE. Smaller organizations did more cross-training, so that if circumstances prevented team members from being present, other staff members could 
cover for them as much as possible. "We cross train everyone because we just believe it's important. You never know what's going to happen, what kind of disaster or emergency might happen and you're going to need different staff' (PACE Administrator, H7).

An overall finding was that the level of participant-centeredness of the PACE program makes it difficult to train IDT members about how to deal with particular cases and team situations. PACE programs are responding to several contexts in defining the right plan for participants, and there are also the contextual realities of the day center, the program itself, and the unique context for the participant in their care setting.

The problem is in the context, so in theory we have over 400 different contexts. Everybody's life is different and every circumstance is different. There could be a 55-year-old that is so ill and otherwise nursing home eligible and there could be an 80-year-old at the other end of that who is a good 80-year-old who really has her mind and requires less care than the 55 year old. So we're going to go forward, after we've taught and educated. So if you could just say that this is always the case or never the case, but it's so contextual, it makes teaching almost impossible (PACE Administrator, G6).

In order to achieve any level of interdependence within the organization, the teams train across professions, teams, and services. The trainings are intended to give new staff members a sense of place in the organization and within their 
team. Interviewees believe that the greater the awareness an individual has about the other services and professions, the more likely they are to bond with their teams and share responsibilities appropriately.

I think it's important for a new person to be exposed to the full range of [services] and not just get sort of acculturated to the site that they are going to be working at. We also have some staff that are consistently shared, that always flip their time; like my role, some therapy staff, other supervisors, there are nurses that float between sites. So we do have staff that work at multiple sites. I think that's helpful. We do also sort of I don't know I just pitch in with each other when needed. An example, right now I have a staff member out on leave, but one of the other sites is a little over staffed right now, so a couple of their staff are floating over to help out. We do as discipline groups, especially support each other and float around as needed (PACE Administrator, I2).

However, just providing the opportunity to learn and train in different areas is not enough. The training needs to be revisited and contextualized as the individual, team, and participant load changes. "I said when we first started doing this, I had clearly said 'please don't just think that when you in-service someone on how to do it, that it's going to follow through. You need to have to check-ins. You need to constantly check in with them,' I say have them be watched, be rated and follow up in their context" (PACE Administrator, X2). Administrators and IDT members both emphasized the importance of keeping IDT members current 
on regulations and regulatory changes. Details of regulatory changes and updates would not just provide new forms and procedure information, but provide explanations, interpretations, and relevance to the everyday job of providing care.

I made sure that everybody had a PACE regulations manual. Everybody had it and everybody read it and we went through it together so there was no question of what the regulations stood for. That is part of our continued education. I have incorporated the people that we have to be part of the process. It makes a big difference. We aren't there yet, but I think we have to get our IDT people to be very independent, even in looking at regulations. We will bring in the interpretations of the guidelines, but allowing them to push the envelope with questions. Does this mean this is what we are doing? How does this data meet this regulation (PACE Administrator, N1)?

However, IDT members have difficulty justifying the time required to read or attend training on regulatory issues. There is not a demonstrated interest in regulatory reasoning by IDT members overall.

Sometimes I actually try to bring to the team a set of regulations where it's very vague, because [the regulation] will say you do A, B, and C and then at the very end it says, "As appropriate for that person." I think when they're looking for direction that that is probably when they are most interested in hearing about the regulations, policies, and the procedures. 
When I speak with them, I remind them how it [the data] all comes together. The teams are not interested in other things except how can they provide for the participant (PACE Administrator, F6).

Cross-disciplinary trainings happen, but nearly all sites expressed a sense that more should be done. Many activities require IDT members' time. IDT members have multiple PACE meetings, continuing education credits, and participant care that together fill up the IDT workdays. Finding the time to conduct further trainings is a challenge.

Most of our trainings I think are across disciplines and I think wherever possible we try not to do trainings just for one discipline, although that has to happen too because we certainly have our own jobs to do. Most of our training in the past couple of years has been a lot of internal processes that need updating and we all have to get trained on that kind of stuff. It's solidarity in knowing that we've all got to learn something new that's not necessarily professional development but something essential for operational success. I think there should be more (PACE Administrator, B4).

Procedural elements support IDTs through recruitment and retention, time and space, and training and education. Recruitment and retention are central to a PACE program's successes but continue to create challenges for programs, especially with decreasing numbers of providers with geriatric experience and 
retention issues with entry-level staff. Time and space supports suggest that less is more, and smaller spaces lend themselves to more collaboration; however, they can also lead to frustrations and a shortage of treatment and meeting spaces.

Training and education support IDTs by facilitating interdependence of team members and IDTs with the organizations. Interviewees' sites struggle with making time and resources available to conduct such trainings. Table 4.5 summarizes the findings for the categories of supports for relational elements and the mechanisms that are at work within the PACE context.

Table 4.5: Procedural categories of supports

\begin{tabular}{|l|l|}
\hline $\begin{array}{l}\text { Emerging } \\
\text { categories } \\
\text { of support }\end{array}$ & \\
\hline $\begin{array}{l}\text { Recruitment } \\
\text { and } \\
\text { retention }\end{array}$ & $\begin{array}{l}\text { - New hires are carefully selected to meet PACE criteria and for the team. } \\
\text { - Geriatric shortages in nearly all disciplines make filling vacancies } \\
\text { difficult. }\end{array}$ \\
- PACE regulations are strict on the qualifications of individual who are \\
members of IDTs.
\end{tabular}




\begin{tabular}{|l|l|}
\hline $\begin{array}{l}\text { Training } \\
\text { and } \\
\text { education }\end{array}$ & $\begin{array}{l}\text { - Facilitating interdependence amongst the IDT and the organization starts } \\
\text { in orientation and cross-training to understand the multiple roles with the } \\
\text { IDT. } \\
\text { - Logistics in scheduling trainings are a barrier. }\end{array}$ \\
- It may be impossible to train or adequately prepare IDTs for the context of \\
each participant. \\
$\begin{array}{l}\text { - Many IDT members float to other teams to help cover when staffing } \\
\text { shortages arise. Cross-training may occur over different PACE sites. } \\
\text { - Administrators try to bring more understanding of regulatory reasoning to } \\
\text { the IDT, but IDTs overall think regulatory issues are the administrator's } \\
\text { domain. }\end{array}$
\end{tabular}

\section{Summary of Findings}

The examination of interdisciplinary team environments provided a greater understanding of the complexity of legitimizing team-based practice within current institutional structures. This study addressed the gap in understanding what institutional elements play a part in legitimizing the collaboration of health professionals and health workers as a benefit to patient care. These elements can also create barriers that can affect a team's ability to provide care. Since PACE legislation mandates and prescribes the IDT, the policy-regulative element should be relatively constant among PACE programs.

The question answered by this study was: What elements of institutional context support the use of IDTs and legitimize team practice for the care of frail older adults? The answers were analyzed using the five primary elements for team care outlined in Chapters Two and Three. The framework for this study was developed from W. Richard Scott (2008) and Scott Reeves (2010) to address possible contexts that are external and internal to the team. The supports provided through institutional context presented in this 
chapter were consistent across sites regardless of variation. This suggests that there is a role for standardizing practices in legitimizing and sustain team-based practices.

Several external influences exert power over IDT function. Under the policy and regulative element, regulatory bodies, resource allocation, and quality were the primary issues that created support. The cultural-cognitive element highlighted the supports of interdependence, demography and organizational structure. Within the normative element, participants most frequently suggested organizational values and mission, leadership, and professional boundaries as facilitators of support. Under the relational element, roles and communication were the primary issues that created support. Finally, the procedural element highlighted recruitment/retention, time/space, and training/education as the primary support mechanisms. Table 4.6 summarizes the primary categories of support discussed in the interviews with PACE administrators and IDT members. These findings suggest a series of conclusions and possible questions for future research, which are addressed in Chapter Five. 
Table 4.6: Institutional element support for IDTs

\begin{tabular}{l|l}
\hline Element from literature & Categories of support from the research \\
\hline Policy-Regulative & - Actions of regulatory bodies \\
& - Resource allocation \\
\hline Cultural-Cognitive & - Interdependence \\
& - Demography \\
& - Organizational structure \\
\hline Normative & - Leadership \\
& - Professional boundaries \\
\hline Relational & - Roles \\
& - Communication \\
\hline Procedural & - Recruitment and retention \\
& - Time and space \\
\hline
\end{tabular}




\section{Chapter 5 Discussion, Conclusions and Recommendations for Future Research}

\section{Introduction}

This study examined the institutional context that supports interdisciplinary teambased care for older adults. It is among the first studies to examine the team environment at a macro institutional level and to consider the unique characteristics of the challenges of caring for an aging population from a team perspective. Meeting the health care needs of the growing aging population through alternative workforce models has been the focus of many studies; of particular interest to researchers is the question of how the growing aging population will affect the workforce providing care for the elderly (Baxter\& Markle-Reid, 2009; Bragg \& Hansen, 2011; Miller, Booth, \& Mor, 2008; IOM, 2008; Stone, 2004). There are persistent recruitment and retention issues in geriatric specialties of most disciplines (Coogle, 2005; Miller, 2008; Stone, 2004; Warshaw, 2003), including medicine, nursing, allied health, and paraprofessionals (Bragg 2010; Gawande, 2007; IOM 2008; Stone, 2004). The present study focused on how to support IDT practice in order to advance understanding of the issue of the effects of institutional context.

The propositions stated in Chapter Three were that policy-regulative, culturalcognitive, normative, relational, and procedural elements would support and legitimize IDT models of care. Some findings fell in line with what was expected, but there were some unanticipated responses as well. This chapter will discuss the findings by element and supporting categories, and then highlight policy implications of the findings. Limitations are discussed, followed by several recommendations for future research in 
this area. Table 5.1 summarizes the elements and supporting categories previously discussed in detail in Chapter Four.

\section{Table 5.1: Institutional Elements and Supporting Categories Discussed in Chapter} Four

\begin{tabular}{|c|c|}
\hline Element from literature & Supporting categories based on the research \\
\hline Policy-regulatory & $\begin{array}{l}\text { - Actions of regulatory bodies } \\
\text { - Resource allocation } \\
\text { - } \text { Quality }\end{array}$ \\
\hline Cultural-cognitive & $\begin{array}{ll}\text { - } & \text { Interdependence } \\
\text { - } & \text { Demography } \\
\text { - } & \text { Organizational structure } \\
\end{array}$ \\
\hline Normative & $\begin{array}{ll} & \text { Organizational mission and values } \\
\text { - } & \text { Leadership } \\
\text { - } & \text { Professional boundaries } \\
\end{array}$ \\
\hline Relational & $\begin{array}{ll}\text { - } & \text { Roles } \\
\text { - } & \text { Communication }\end{array}$ \\
\hline Procedural & $\begin{array}{ll}\text { - } & \text { Recruitment and retention } \\
\text { - } & \text { Time and space } \\
\text { - } & \text { Training and education } \\
\end{array}$ \\
\hline
\end{tabular}

The literature review in Chapter Two showed that macro and microstructures are significant in defining team effectiveness, efficiency, and ability to engage in a collaborative care process. Institutional structures are key players in sustaining and supporting team-based practice. The history of the U.S. health system is a highly political and professionally dominated narrative, rife with power, legitimacy, and quality struggles for care providers and patients alike. Health delivery systems in this country have grown up around policies and payment structures that are fragmented, isolated, and not collaborative in nature. Moreover, these structures and processes do not provide the adequate quality of care that is necessary for long-term or end of life care. Complex cases of the frail elderly require a new approach and different means of delivering care 
and defining quality. This qualitative study has identified institutional context elements that support IDT in caring for older adults. Through the interviews of 32 PACE staff members from seven programs across the U.S., the complex reality of IDT context has been highlighted. PACE programs provide an interesting case study of IDTs because they are required by legislative mandate to use a team-based approach to care for frail older adults. Health care organizations, providers, and participants are all interconnected and interdependent in making health care decisions. The context of health care institutions influences choices made around health care delivery models and how that affects organizations, administration, policy, and practice. The use of IDTs to deliver quality, cost effective, person-centered care to frail older adults requires on-going legitimization efforts at the individual, organizational, and institutional levels.

Addressing workforce issues in long-term care and geriatrics in general will require a reorganization and reconsideration not only of the health care delivery system, but also of provider training, regulation of care delivery and quality. The literature indicates that to meet the needs of frail older adults there will have to be an approach to care delivery with an interdisciplinary and interdependent approach that involves a broad spectrum of stakeholders. Health care stakeholders are moving towards meeting the demands of a health care workforce that is prepared for the challenges and new ways of providing patient care including home and community-based, patient-centered and teambased approaches. These statements support the need for research that examines institutional issues that affect the delivery of care using an interdisciplinary team approach (Ducanis \& Golin,1979; IOM, 2008; Reeves, 2010; Zeiss \& Steffen, 1996). 
This section will review each element described in Table 5.1 and provide recommendations pertaining to each supporting category.

\section{Policy-Regulative}

The policy-regulative element speaks to the rule-setting, monitoring, and sanctioning activities engaged in by the respective institution. Teams have competing interests, which require mediation by rules and referees to ensure order. The third parties must be neutral or the outcomes will be influenced by their behavior and may not serve the intended end (North, 1990). The policy-regulative element of institutions renews interest in the role of the government to be rule maker, referee, and enforcer (Skocpol, 1985). Many rules and laws are ambiguous enough that, contrary to common belief, there may not be a clear code for conduct. Policy-regulative elements were examined in an attempt to identify policy mechanisms and regulatory practices that affect the ability of the team to collaborate and work together. Three primary policy or regulative factors were identified (see Table 4.2): the actions of regulatory bodies, resource allocation decisions, and quality metric definition. In the following section the significance of each element is discussed.

Regulatory bodies. PACE is a heavily regulated program at the federal and state levels. The long history of program development and funding streams provide PACE programs with legitimacy as a provider of LTC for older adults. The initial intent of CMS in 1994 when demonstrations of the PACE model were approved was to proliferate PACE across the country (CMS, 2006). That growth has not been realized and the number of PACE programs consistently falls below legislative caps. The Balanced 
Budget Act of 1997 authorized 180 nonprofit PACE programs plus ten for-profit demonstration programs. By early 2004, only 39 programs serving about 10,000 individuals (and no for-profit demonstrations) were operating (Gross, Temkin-Greener, Kunitz \& Mukamel, 2004). In PACE Medicaid legislation, caps of how many PACE programs could be started up were limited as appropriate state-by-state. As of January 2013, there were 94 PACE programs nationwide, and many legislatures are lifting the caps, as having too many programs does not seem to be an issue (NAP, 2013). The lack of programs raises questions about the ability of the PACE model to scale up to a widely available model of care that can meet the needs of the expanding elderly population. CMS and states still support PACE program growth, but would now like current programs to diversify the model and serve a broader scope of the population. However, the regulatory burden makes this difficult even for large PACE organizations. The capacity for PACE organizations to grow and diversify is capped by rules that do not match the workforce and participant realities.

The literature review suggested that waivers would play a large part in customizing PACE services and workforce configurations to the varying organizational contexts, as the Medicaid waiver has been an instrumental tool to customizing the Medicaid program to state contexts (Thompson \& Burke, 2010). However, in the past few years, most waivers requesting changes in team composition and primary care privileges for nurse practitioners were denied as discussed in Chapter Four. Most of the waivers granted were related to day facility licensing, since those requirements are 
duplicative with PACE regulations. Thus waivers were not found to have the impact that had been anticipated.

PACE programs are audited every two years for the first five years of operation and every two years after that. The audits do not interfere with the day-to-day provision by the IDT. However, the approach and results of the audit do affect IDT functioning. Administrators and IDT members alike agreed that the methods of examination did not adequately consider the core principles of PACE IDT services. Auditing methods vary widely; some audits by federal and state regulators did include observation of meetings, home visits, and interviews with staff. Other audits focus almost solely on care records.

In order to support IDTs as a care delivery model, regulators need to approach audits from a collaborative standpoint. It is essential that federal and state bodies define jurisdictions and share results in order to reduce confusion of being graded on the same item in different ways. Regulations should also begin to allow IDT structures to be more flexible, allowing more variation in team composition and an expanded role for the nurse practitioner. Greater flexibility does not suggest that services will no longer be available but that the services can be tailored better to meet a participant's needs, since not all participants need eleven team members.

Resource allocation. The all-inclusive provision of PACE and its capitated payment structure scheme puts the IDT in a position of decision-maker for services similar to the role of an insurer. The IDTs decide on care plans to approve or deny particular services to a participant and are supposed to be sensitive to cost accrued by 
particular participants. This is a unique aspect of the PACE program. First, the team selects services urgently needed by the participant. Once the participant's urgent needs are met, care focus shifts to prevention and independence. Most IDT members are not trained to make service decisions in concert with other providers, and may not understand cost implications, which would be a primary concern for an actual insurer.

Over time, participants realize that the rules of service provision are not fixed for PACE. Many participants and families may request services or equipment that are not necessarily needed, but would improve the quality of life of the participant. The responsibility falls to the team to decide whether to provide the service or equipment. They do so with the input of the participant and their family (ensuring participantcentered care) but must also weigh the resources of the program. If participants are not happy with a decision, every PACE program has a grievance process by which the participant can appeal to a higher authority, usually the administrative office at the state level. Administrators engage with the process at the state level as well, facilitating resolution with dissatisfied participants and families

Administrators said that the IDT ideally would be isolated from financial matters and focus solely on participant care; however, the decisions related to participant care are inextricably tied to financial decision-making. IDTs are often mindful of the financial realities of the all-inclusive care provide by PACE. The financial realities exclude the valuing of preventive and collaborative care, as these are not attributed quantitative value in the PACE capitation rate calculation. IDTs and administrators conveyed an understanding that preventive and collaborative care is the foundation on which PACE 
quality of care is built, but within current financial calculations, these hallmarks are not attributed adequate value.

In order to support IDT practice, organizations must bring a level of transparency to the cost and value of services provided by IDTs. Although the PACE program is "allinclusive", there are limits on what services should be provided, and these limits often go unrecognized until they are crossed. The organization can assist with IDT decision making through policy and procedures that help define the limits of services. IDTs can also learn from each other on where their experiences have found those limits to be, and together these can begin to draw up a framework for helping IDTs determine where a "need" crosses over and becomes a "want."

Meaning of quality. Among health care providers there is little consensus on how to weigh the various attributes of health care quality (Ransom, 2008). However, the need to focus quality around responsiveness to participant preferences is increasingly being acknowledged since the IOM included 'Respect for patients' preferences and expressed needs" as one of the six aims of its Quality Chasm report (2001).

The core principles of providing all-inclusive care by a collaborative IDT to maintain independence, according to interviewees, are not actualized or validated through the current acute care focused measurements for quality. PACE IDT services are so customized to an individual that it becomes difficult to use these metrics in a way that is meaningful to improve care. The interpretation of quality data and its utilization is the providence of the administration; how and when the information is shared is highly 
variable. The quality of services can be addressed at the team level; however, there are many providers that may not be included in the IDT meeting or other communications regarding quality. In many cases, the aides and assistants would primarily be informed if there was a complaint or quality issue and the issue would be handled outside the team. This adds to the disconnect between IDT meeting attendees and those who are not required to attend.

Timeliness of service was another dimension not adequately represented in current measures. Many IDT members felt that the rate at which they were able to fulfill a need was an important concept. Timeliness is included in the six aims for improvement in the IOM Quality Chasm report (2001); the IOM defines timeliness as reducing the waits and delays for both those who receive and those who give care. Interviewees felt that this measure is not highlighted enough through the organization or other regulatory bodies.

There is an increasing emphasis on patient-centered care, but it is unclear that current broadly standardized quality measures will be adequate to capture the role of the patient in the decision-making process. Patient-centeredness adds a layer of context that interviewees felt is not accounted for adequately in current measures. Interviewees questioned if standardized data can be made meaningful in highly contextualized settings, and can be made useful to providers and participants alike.

Synthesis of policy-regulative findings. Policy and regulatory practices discussed in this section constrain and systematize behavior. PACE IDTs experience 
those constraints and systems through regulatory body actions, resource allocation, and quality measurement. Regulatory bodies need to remain flexible and improvementfocused to best support IDTs in providing all-inclusive patient centered care. Resource allocation requires a sufficient amount of communication from the organization to the IDTs in order to provide guidance on provision and denial of services. Quality measurements should reflect the same level of contextualization that guides service provision by IDTs.

\section{Cultural-Cognitive}

Cultural-cognitive elements of institutions are shared conceptions that constitute the nature and social reality and the frames through which meaning is made. Meanings arise through interaction and are maintained and transformed as they are used to make sense of what is going on. To create understanding of a situation, one must take into account the empirical conditions as well as the administrator's and team member's subjective interpretation of those conditions. Scott hyphenates "cultural-cognitive" as a singular element acknowledging that the internal interpretation processes are influenced by the external cultural frameworks (2008a). Cultural and cognitive elements were examined in an attempt to identify particular issues that affect the ability of the team to collaborate and work together. Three primary cultural or cognitive supporting categories were identified: interdependence, demographic characteristics, and organizational structure. Within each of these supporting categories, more specific examples were identified and detailed how those examples supported or created barriers for practice. 
Demographic Characteristics. The IOM report Retooling for an aging America: Building the health care workforce (2008) stated that with few exceptions health care workers at all levels need greater training on the unique needs and complex issues of caring for older adults. The IOM recommended that licensure, certification, and maintenance of certification for all health care providers should include a demonstration of competency in the care of older adults (2008, p.7). The complex needs of the elderly have been crucial in legitimizing team-based care. The investment of federal and state governments through Medicare and Medicaid creates more interest in how the use of teams can be more efficient and effective than traditional models of care delivery. However, it is widely acknowledged that aging does not create a standard level of frailty. Frailty can be a characteristic of physical or mental well-being. The acuity of these individuals is highly variable; however, there is no wavering on the number of contact hours per participant in PACE programs. Use of fluid communication systems among IDT members and the participant could allow customization of contact hours for each participant. PACE participants on the whole are getting younger, according to those interviewed. If this trend continues, there may be greater support required to move teams form a medicalized approach to a more social and mental health focus. Teams and administrators alike referred to mental health services as a deficiency in their programs; adequate resources were not available to meet these needs.

Socioeconomic (SES) factors play a large role in PACE as most of the participants are Medicaid eligible. Leatherman and McCarthy (2005, IOM 2008) found that SES plays the biggest role in determining the use of preventive services. By the time 
many individuals enroll in PACE, many health conditions have been neglected and have become compounded into more complicated health issues. The complex cases require an IDT for the range of medical and social interventions required. The nature of the complexity of participants that enroll in PACE creates a sense of isolation from the parent organization and mission in some larger programs. Between home visits and day center hours, some IDT members felt a greater connection to the culture of the community they serve than to the parent organization (Bosch, 2008). Parent organizations may provide administrative support and resources, but many interviewees at day center sites located at a distance felt not as relevant or important to the parent organization, as those day centers located in closer proximity to the parent organization. Therefore, even a single PACE program can have great variation in cultures exhibited throughout its multiple day centers.

Even though PACE enrollees are required to need nursing home level of care in order to qualify for services, the goal of the program is to foster independence as much as possible. This creates a greater role for the enrollee than in traditional LTC settings. The enrollee should engage with their care in order to improve their overall health and foster greater compliance with treatment regimens. This can prove challenging for IDT members, as the level of compliance may not be as great as in other settings. However, the IDT can support those working with the enrollees in the home and find creative solutions to improve commitment to the treatment regimens. Moreover, interviewees emphasized the importance of engaging the participant as the center of the IDT. 
Interdependence. In assembling an effective IDT, where the team is focused on people with complex health and social care needs, there is an expectation that the IDT will collaborate to optimize care for the participant (Onyett, 2003; Tannenbaum, 1996; West, 1998). IDTs persistently struggle to understand the roles and scope of practice of all of the different members of the team. The IDT has some legitimizing authority of its own. The IDTs in PACE programs act as their own advocates to justify their activities. Interdependence legitimizes teams through adding role definition and providing support on professional and personal levels to avoid burnout.

Interdependence amongst team members is critical to team support. The inclusion of participants requires a development of interdependence of the IDT and the participant. As discussed in the resource allocation section in Chapter Four, the distinction between 'needs' and 'wants' is becoming less clear for participants, and what a participant feels is a need may not be considered a need by the IDT. Improved interdependence and inclusion may help clarify these terms for both parties. Interdependence can also occur at the level of the approach to care. PACE, hospice, and palliative care have many similarities in their approaches to team-based, participant and family-centered care. Organizations that offer hospice and palliative care have found fruitful interorganizational partnerships that support all programs and their teams. PACE programs tend to function in isolation due to their all-inclusive care approach, but there may be real benefits to improving the visibility of PACE through its connection to other established, respected services through interorganizational partnerships. In a world 
where few team-based models are used, IDT members feel that this would also provide greater support and examples of teamwork.

The care provided by an IDT is intense, and an interdependent culture may help prevent burnout, conflict and job/service dissatisfaction within the IDT and with participants and family members. However, as discussed previously in Chapter Four, all members of the IDT need to be included in the definition of interdependence, including entry-level staff who are frequently excluded from team communications and supports.

Organizational structure. All PACE organizations experience a level of isomorphism with environmental institutions, from the early legitimization of the On Lok model to subsequent demonstration sites. From those demonstration PACE programs, criteria for assessment, program details, and a dependence on Medicare and Medicaid funding have promoted a level of stability and quality that is consistent across programs (Meyer \& Rowan, 1991). The PACE model is supported in various types of organizations. Through the isomorphic qualities, or "cultural inheritance" as one interviewee put it, the IDT is assured of external legitimacy. However, the On Lok model may not be sustainable in all markets. As discussed in Chapter Two, On Lok and the demonstration sites were all located in urban areas with greater access to health care workers. The supply of health care workers greatly affects the communities' ability to staff the IDT within the PACE program. Through Medicaid, states have some power to approve changes in the PACE program through waivers and other request mechanisms. However, few programs have successfully petitioned for changes to IDT requirements. These programs have found other ways to reconfigure the IDT within the prescribed 
structure, but in a way that allows the program to be responsive to workforce realities and participant needs.

Larger health systems and long-term care structures are invaluable in providing support and resources for PACE programs and their teams. One example of this type of support is the utilization of lobbyists for the health systems' interests (PACE included) in the state legislatures. PACE programs are increasingly developing government relations staff or contracting with lobbyists in order to represent and track the programs' interests in state capitols during legislative sessions. Many of the larger organizations see having a PACE program as furthering their own mission. However, the PACE programs that were interviewed were generally left to their own devices to administer and find supports for the program and its workforce, independent of services and programs offered in the parent organization. Freestanding PACE programs are very few in number and the necessary administrative structures for sustainability are missing.

Synthesis of cultural-cognitive findings. Cultural-cognitive elements mediate between an IDT's external environment and the response of the IDT to that external environment in order to make sense of what is happening. PACE IDTs create meaning through their interactions with their external environments through interdependence, demographic characteristics, and organizational structure. For IDT support, an interdependent culture must exist amongst the team members and at the organizational level. Interdependence fosters greater understanding, assistance, and legitimacy to sustain and improve IDT practice. Care planning by an IDT depends greatly on the characteristics and culture of the participant population. The unique demography of 
PACE participants can also influence the culture of specific day centers. Organizational structure creates a very specific system of support for IDTs that lends a level of stability for IDT practice.

\section{Normative Elements}

Normative rules introduce a prescriptive, evaluative, and obligatory dimension into social life. Norms specify how things should be done. Some values and norms are applicable to all members of a group, whereas others apply only to selected types of actors or positions. The underlying normative concept remains the focus on social obligation for performance. Organizational and team norms were examined in an attempt to identify particular patterns of behavior that affect the ability of the team to collaborate and work together. Three primary normative factors were identified: organizational values/mission, leadership, and professional boundaries. Within each of these supporting categories, more examples were discussed and described how the normative factors supported or created barriers for practice.

Organizational mission and values. PACE is a mission driven program, and all interviewees made it clear that above all else their goals are to keep their participants independent as long as possible and provide all-inclusive care in a participant-centered way. IDTs are so participant-centered that sometimes the decision-making becomes more about keeping the participant happy than about medically or socially necessary interventions. 
The nature of the care provided by the IDT is more about participant well-being than finding any cures or regaining a previous level of function. This decision-making process is counter to traditional medical decision-making. Harm reduction is commonly used in social services, and is a paramount approach within PACE IDTs. Larger parent organization mission and values can complement the IDT's decision-making process with infrastructure, housing support and community resources aimed at keeping elders independent. PACE aligns with the new focus of reducing hospitalizations and nursing home readmissions. With the elderly population PACE IDTs care for, hospital and nursing home placements are going to occur. As discussed previously, the focus of the IDT becomes getting the person back to their home with necessary adaptations as quickly as possible.

The IDT meeting is the central forum for the administration to reinforce the mission and values. Many teams start with a restating of the mission and values at the beginning of the meeting or have them posted around the day center and meeting rooms. Such visibility reassures the IDT that even though it uses a non-traditional decisionmaking model, these are the priorities. The participant comes first. This emphasis provides the opportunity to emphasize that social needs are just as important as medical needs. Participant self-determination is empowering for participants and the IDT alike. It also has drawbacks for practitioners, as practitioners cannot assume that participants are more compliant or follow instructions for treatment regimens as closely as the IDT might hope. 
Leadership. PACE IDTs are supported by leadership structures that are informally located within the team and formally along disciplinary lines. Each discipline has its own supervisor (a few disciplines are grouped); thus the supervisory structures of a collaborative and horizontally managed IDT are vertical discipline silos. When issues arise, most administrators expressed that they could be handled or worked out amongst the IDT. If they cannot, then the issues are taken up at a discipline-specific level. This can create ripples of non-interdisciplinary work within a day center. A discipline-specific supervisor must supervise most professional health disciplines, but few PACE leadership structures were as collaborative as an IDT. Two of the sites in this study mirrored the IDT model in leadership. The layers of administration primarily support the IDT, by seeing to regulatory reporting, handling grievances, and facilitating the IDT to work together. The administrators act as a bridge among the federal, state, and parent organizations and the team. Quality improvement, policy information and changes are relayed to the team by the supervisors and it may be up to them to determine what the IDT "needs to know."

Informal leadership bridges the participant, family, and IDT. Since the participant and family are not present at every IDT meeting, a member of the IDT who is familiar with the participant and family will advocate on their behalf at IDT meetings. A community nurse or social worker typically fills this role, but interviewees also reported OTs and PTs filling this role.

Physicians are still the default leaders in IDTs. The PACE regulations support a strong physician leadership position and many decisions cannot be finalized without a 
physician's signature. Interviewees describe the physician leadership role during the meeting as central to defining any other leadership roles. A disengaged physician may not share decision-making power except to "placate co-workers." However, in most cases, the physician was involved in engaging and empowering team members, and actively deferring power in non-medical decision-making to those topical experts. Several interviewees stated that if the physicians do not "get on board" with the IDT concept, they typically do not stay with PACE. Due to the lack of attendance by the drivers and personal care assistants (PCAs) at team meetings, there are little to no leadership opportunities for these staff within the IDT. The leadership role may generate greater investment in the work of entry-level staff, but the IDT participation by proxy strips that potential away for PCAs and drivers.

Another visible and empowered leader within the IDT is the meeting facilitator. The facilitator in some cases can supersede a physician in recalibrating meetings back to a collaborative process. They can also go the opposite way, by deferring to the physician for input, and reducing the level of collaboration in the IDT meeting. The facilitator's role is to keep the meeting flowing and make sure everyone's voice is heard. Interviewees expressed that some facilitators were having difficulty keeping the meetings on topic or allowing those with something to contribute to have their say. The training and preparation facilitators receive is highly variable amongst PACE programs. Some organizations provide formalized training schedules, practice and shadowing opportunities, while other programs start the facilitator in the meeting and hope they figure it out for themselves. Considering the significant role the facilitator plays in 
meeting efficiency and productivity, PACE organizations should consider a more standardized training and preparation of facilitators.

Professional boundaries. As discussed in Chapter Two, social identity theory (Onyett, 2003; Tagfel \& Turner, 1979) suggests that people derive a sense of identity through group membership and intergroup comparisons. Members of an IDT are simultaneously members of their professional group and their IDT. It is particularly true within PACE programs that the professional group and the IDT will define the boundaries of practice very differently. Every state establishes its own scope of practice law for licensed professions; however, the role may be formally redefined by the PACE federal legislation or informally by the IDT (the latter is discussed in the following element). An example is the nurse practitioner, as discussed earlier; the state scope of practice may allow greater autonomous privileges to an NP in private practice than to an NP working in a PACE IDT within the same state.

Team communication and cohesion is very important in defining boundaries in such a contextualized service model. The role and internal boundaries for each IDT member can change depending on participant need. Different disciplines will have greater authority depending on their expert topic areas. One consistent area mentioned in interviews was the definition and implications of patient safety. The IOM (2000) defines patient safety as "freedom from accidental injury." The disciplines agree on the importance of patient safety, but will often disagree on the optimal way to realize a safe experience for participants. These disagreements tread on boundary issues as to who has the authority to define what is safe for a particular participant. An interdependent culture 
can ease definitions and interpretation, as all IDT members may better understand the point of view of their respective disciplines and team members. Over time, interdependence may lead to cognitive shortcuts of understanding boundary issues. As disagreements reemerge, a team that has been through the process of boundary definition may default to the agreement worked out previously. For example, if there was an OT who supervised the installation of handrails throughout a participant's home, then the next time a similar safety issue occurred, this responsibility could default again to the OT in safety matters of the home since s/he has addressed the safety need in this way before.

The final boundary category is the formality of the professional scopes of practice. Non-medical staff must familiarize themselves with the various team roles and send participants to the right place to get medical advice if they feel unwell while at the day center. Informal suggestions or advice cannot be issued. All medical advice must come from the appropriate professional, including something as simple as suggesting taking an aspirin for a headache.

Synthesis of normative findings. Normative findings in this section not only constraint behavior but also confer rights and duties of IDT members. Organizational mission and values support PACE IDTs through legitimizing the patient-centered care planning as provided by the IDT. Leadership structures are consistently segregated by discipline to support IDT practice leadership structures should also be interdisciplinary and collaborative. Professional boundaries are set at the normative level by state scope of practice regulation. Scopes of practice overlap between disciplines and a greater 
interdependence of disciplines can further delineate professional boundaries that best serve the team and the participant.

\section{Relational Elements}

Relational elements center on the factors that directly affect the relationships among health professionals. Organizational and team relationships were examined in an attempt to identify particular issues that affect the ability of the team to collaborate and work together. Three primary relational factors were identified: roles, communication, and team structure/development. Within each of these primary factors, examples that are more specific were identified and described to illustrate how they supported or created barriers to practice.

Roles. The formation of professional roles is an essential element of effective IDT relationships (West \& Markiowicz, 2004). Clear roles help define the nature of each member's tasks, responsibilities and scope of practice. Typically, roles are considered more of a normative element under professional boundaries, but they are a relational characteristic that emphasizes that social definitions sometimes trump external definitions of roles. Internally perceived competence can redefine one's role within the IDT. This may restrict the scope within which individuals practice.

Many teams create roles that are beyond the discipline-specific titles. One role in particular that came up many times is "the bridge" or "the glue." This refers to one person who may not be a formal leader but is a confidante and advisor outside of the IDT meetings, and displays skill at facilitating interdependence and seeing situations from 
multiple perspectives and helping others to do the same. Other roles mentioned include "the agitator", who constructively disagrees in order to promote creative problemsolving. "The advocate" is the person on the IDT who will stand up for the participant and family requests. Finally, there is "the extinguisher" who is someone who can calm intense arguments by effectively restating the problem and the various points of view. The role titles have been consolidated for easier reporting in this document, as there were different titles on different teams, but many interviewees described all as central to positive IDT relationships.

Another socially derived role distinction relates to those IDT members who visit the participant's home and those who primarily work in the day center and clinic. The interviewees reported experiencing different perspectives of a participant, depending on where they primarily interacted. They may see the same participant but the two environments can affect the participant's reality and behavior in very different ways. The day center is a clinical setting, with spaces for socialization. As reported, participants tend to exhibit different conduct in the day center than in their home. At home, they can act quite differently and it can be the role of staff that make home visits to keep the IDT informed and current with the home reality. This can affect the care plan if it does not match the behavior in the day center.

Physicians maintain control over their role. PACE regulations, as discussed previously, prevent NPs from acting as PCPs. It is up to the physician to relinquish some authority within the IDT. If a physician does not relinquish some authority to the rest of the IDT, either verbally or explicitly, it will be detrimental to the IDT and participant. In 
particular, physicians must relinquish some authority in non-medical decision-making. There is a great relational cost to an IDT member not acting collaboratively - both to the IDT and potentially to the participant. Management and leadership roles are also socially constructed to some extent. Many supervisors intervene in IDT business only as needed. IDTs are left to do their work until they request training or require advice. The role of a supervisor can change depending on how they are engaged by the IDT.

Communication. Communication within IDTs occurs in a variety of verbal and non-verbal forms. Central to IDT communication is the meeting. The frequency varies; some have a meeting every day and some once a week. IDTs also communicate via electronic health records, but many found the records ineffective for long-term teambased care. Written notes in the IDT meeting room, email and instant messaging are frequently used. Open and free-flowing communication among team members is critical in order to deliver effective care.

As mentioned in other elements, there are few avenues for drivers and PCAs to communicate with the IDT directly. The primary way is by proxy through the supervisors. This communication may be face-to-face, or through hand-written notes or email. The people who see the participants most often in their homes report most often when a situation becomes urgent, which for a program like PACE can be too late for effective remedial action. The value added by drivers and PCAs can support preventionfocused observations around changes in routine, favoring a recurring ache, or increased isolation, which do not currently have a consistent pathway to the IDT. One administrator highlighted that the drivers and PCAs may not entirely understand how 
valuable their contributions could be to the IDT. Thus, it would behoove administrators to integrate more individual PCAs and drivers, through mandating participation and attendance at IDT meetings.

Conflict among team members can also come from a number of relational issues. Conflict can create a barrier to team relations and performance, but it can be a positive influence as well. West (1994) stated that conflict could be a source of "excellence, quality, and innovation." However, conflict must be handled well if it is to be positive in its contribution to a team's decision-making process. The key to keeping conflict positive in an IDT is, according to interviewees, to keep the focus on the participants and family, and away from specific criticism of IDT members. Facilitators play a crucial role in managing conflict in the course of a meeting and possibly addressing a resolution outside of a meeting. The IDT meeting is at the crux of IDT communication. IDT members must arrive prepared, and engage in active participation and listening. The legitimacy of each discipline is largely defined within the meeting context.

An interdependent culture facilitates the IDT communication flow in and out of the IDT meeting. Clarity of purpose and roles of the other IDT members can also provide individuals with greater confidence when presenting an issue to the group. It can be intimidating to speak at an IDT meeting, as some of them become quite large in terms of the number of people in the room. If an individual knows they have something of value to contribute to the team on behalf of the participant, it may foster broader participation. 
All PACE IDTs are not seamless in their communication all of the time. Some teams communicate very poorly and alienate many members. Some teams report having good communication even with an IDT member who refuses to participate or listen to other members. Being part of an IDT does not guarantee buy-in and respectful communication. However, many of the teams find ways to "muddle through" until the disgruntled individual leaves. There is a fundamental belief of IDT members and administrators alike that those individuals that "don't get it (PACE)" or refuse to work collaboratively with the IDT will self-select out of PACE.

IDT communication contexts are in constant flux with changing participant loads and needs. This also changes with new IDT members, leadership, and facilitators. The IDT should maintain flexibility to allow it to maintain the communication principles that work and keep communication flowing.

Synthesis of relational findings. IDTs consist of individuals who each have a unique way of relating to others on their team. Social constructs within the team affect role definition and communication, which supports IDT practice. Roles are very specific to the IDT composition, and many roles serve to facilitate better relationships amongst team members as well care provision for the participant. Communication is most accurate when received first hand, yet PCAs, drivers, and the participant and family are represented by proxies in order to facilitate participation. Greater investment in engaging all stakeholders and team members could improve relationships amongst team members and between the team and the participants. 


\section{Procedural Elements}

The procedural element focuses on factors that affect how the work is conducted and the routines that happen (or should happen) on a regular basis. Organization and team procedures were examined in an attempt to identify particular aspects that affect the ability of the team to collaborate and work together. Three primary procedural factors were identified: recruitment/retention, time/space, and training/education. Within each of these primary factors, more examples that are specific were identified and discussed how they supported or created barriers for practice.

Recruitment and retention. Recruitment and retention of health professionals to stay in geriatric specialties is a challenge across disciplines. Many disciplines are experiencing a shortage in the professions with geriatric expertise. The search for these professionals is complicated by the fact that PACE regulations stipulate extra qualifications criteria that practitioners at all levels must meet, as discussed in Chapter Two. Different sites expressed different experiences with filling vacancies; some sites cannot find a physician, some cannot find a nurse, and others cannot find therapists.

Where recruitment of professionals is a challenge, many PACE programs struggle to retain paraprofessionals and entry-level staff. Once a PACE program recruits the professionals it needs to fill the IDT, the retention rate is quite good overall. However, PACE programs experience many of the same challenges with retaining paraprofessionals that other LTC services and programs experience. Administrators are mystified by it, but the situation of the entry-level workers is very different from members attending the IDT. When the day-to-day work of the PCA in particular is 
examined, it becomes clear that they do not receive the benefits of working in a team. It is difficult to tell if being a PCA for a PACE program is different from being a PCA in a nursing home setting, for example. Driver retention is better, anecdotally, than PCA retention. Legitimization of the team as a whole can be a struggle for many PACE programs. The benefits of being part of a team are not extended to individuals who are entry-level.

Physicians, even when recruited, have a higher rate of attrition than other professions in the PACE sites in this study. There are many unique characteristics of working in a PACE program and it can be a difficult adjustment for many physicians. Working collaboratively and engaging with other professionals on an equal footing is a challenge for some. Some physicians have reported the salary structure is undesirable when fee-for service seems more lucrative. Overall more physicians were very satisfied with their place at PACE and enjoy working in their teams collaboratively.

Time and space. Time and space are closely linked concepts that affect IDT work. Spatial boundaries can be used to bring services and disciplines together or keep them separate (Armstrong, 1985; Reeves, 2010). Differences in workspace are certainly a concern of PACE programs that need to accommodate a large team in the day center and allow participants to arrive and participate in activities and see many of their IDT members. The compact structure of many PACE buildings can lead to sufficient amounts of informal communication among IDT members, staff, and participants. There are no shortages of opportunities to get distracted while in the PACE day center and even these distractions can be useful in developing interdependent team cultures. 
In the PACE programs that participated in this study, a few had day centers that were specifically created to serve an all-inclusive purpose. Participants visiting the day center as well as receiving all of their clinical services provide a great challenge for many IDTs. Most sites were satisfied with the way buildings serve the purpose of PACE IDTs. Even with so much going on each day, interviewees did not report significant inconvenience. However, some sites experience shortages in service spaces for meeting privately with patients and families, and bathing rooms and toilets.

The overall response of interviewees was that the relatively small spaces facilitated better IDT functioning and communication through seeing both entry-level staff and professional members of the IDT throughout the day. Some sites had mobile team members that work at multiple sites. Lack of co-location of professionals that are in short supply is a persistent issue with some PACE IDTs.

Education and training. A fundamental principle of interdisciplinary teams is that IDTs are an experiential learning process. Learning occurs by doing something, and by actually working in collaboration with other professionals. This approach is in contrast with the didactic instructional method typically used to teach core knowledge content in health professions education. This is changing, albeit slowly. Many health professions schools are integrating schedules of the different disciplines in order to provide a more interprofessional experience (Reeves, 2010).

Interdependence as interdisciplinary culture begins to develop from the orientation of how individuals are trained and introduced to the IDT and its members. 
One IDT member recalled days of shadowing many of the IDT disciplines as a part of the orientation to learn roles and functions, and to begin to understand the nuances of working collaboratively with other professions. Given an appropriate and inclusive orientation to PACE and the IDT, many interviewees said that it is impossible to prepare for the participant-centered care component. What makes this component so difficult is that every participant is different, and the team dynamic can vary depending on which participant is being discussed. It is difficult to train new IDT members to be flexible and prepared for the unexpected. Many IDT members must float across units to cover shortages in other teams. Few of the sites engage in active cross-training to prepare professionals to work at the other sites. Some hold interdependence as an organizational core characteristic. However, most PACE programs adopt a "wait and see" approach, and trust the flexibility of PACE professionals to quickly adapt to other sites.

Half of the administrators interviewed expressed concern at the disconnect between IDTs and regulatory requirements. The day-to-day work of IDTs can be isolated from many of the regulatory tasks. Many interviewees thought it would behoove IDT members to know more about why they collect the data they do, and to understand why, for PACE at least, regulation matters so much. Some administrators provide semi-regular trainings on regulation updates or require each IDT member to have access to a regulation manual; both activities are intended to provide greater context to the data, hopefully to inspire greater compliance with data reporting and follow up.

Synthesis of procedural findings. Procedural support standardizes practices to maintain highly functional teams. In order to support IDT practice PACE organizations 
highlighted recruitment and retention, time and space, and training and education as the primary ways to support IDTs. Recruitment and retention of IDT members are at the core of team function. PACE programs are experiencing workforce shortages and staff retention issues similar to many other geriatric services. Time and space illustrated the benefits and drawbacks to various time demands and physical space available for care. Training and education experiences have been positive overall; however, logistical issues are persistent for IDTs.

\section{Overall Synthesis}

The expansion of interdisciplinary team models will require many of the governing institutions and organizational structures to adapt in order to support teams in a comprehensive and consistent way. This study addressed the gap in understanding how institutional elements play a part in legitimizing the collaboration of health professionals and other health workers as a benefit to patient care. Several external and internal institutional constructs exert influence over IDT function. Table 5.2 highlights the most significant findings for each element as prioritized by the researcher. 
Table 5.2: Most significant findings for each element

\begin{tabular}{|c|c|}
\hline Element & Significant Findings \\
\hline Policy-regulative & $\begin{array}{l}\text { - } \\
\text { - } \quad \text { Stanersight should refocus around improvement strategies. } \\
\text { determination between patients' needs and wants. } \\
\text { - The roles of the family and participant need to be } \\
\text { represented in quality measures. }\end{array}$ \\
\hline Cultural-cognitive & $\begin{array}{l}\text { IDT members can increase team function by improving } \\
\text { the interdependence within their team. } \\
\text { Integration of needed alternative providers is crucial for } \\
\text { IDTs to address the changing needs the PACE participant } \\
\text { population. } \\
\text { IDTs are resource intensive to start up and sustain, and } \\
\text { substantive organizational structures are required to meet } \\
\text { this need. }\end{array}$ \\
\hline Normative & $\begin{array}{l}\text { Participant-centered care permeates every level of the } \\
\text { PACE program. } \\
\text { Leadership of IDTs should reflect the collaborative nature } \\
\text { of the IDT model. } \\
\text { - An expanded role for the nurse practitioner as a primary } \\
\text { care provider could alleviate some the staffing issues } \\
\text { experienced by PACE programs. }\end{array}$ \\
\hline Relational & $\begin{array}{l}\text { Relationships among team members are a critical element } \\
\text { in further delineating roles. } \\
\text { - Front line workers should be integrated into team } \\
\text { communication more directly, possibly by attending } \\
\text { meetings in person. }\end{array}$ \\
\hline Procedural & $\begin{array}{l}\text { - IDTs are a good way to improve retention amongst health } \\
\text { professions; however, front line workers should } \\
\text { experience the same level of team participation in order to } \\
\text { maximize their contribution. } \\
\text { - Physical space is not as crucial an issue as expected. } \\
\text { - Teams are resilient and make due in the spaces they have } \\
\text { to work with; many prefer the smaller spaces. } \\
\text { The meeting burden of teams should be reconsidered to } \\
\text { allow for team building activities and education to take } \\
\text { place. }\end{array}$ \\
\hline
\end{tabular}




\section{Implications for Policy and Practice}

The current health care environment is rapidly changing. Policies, regulations, culture, norms, procedures, and relationships are responding to a shift to patient-centered care and a call to find greater efficiencies within workforce and organizational practices. There is a substantial resource investment required to implement a team-based model. By considering the complexities of sustaining successful team practice, organizations can structure their team environment more efficiently and effectively to support team function. Collaborative interdisciplinary team care embodies several core values put forth in the Institute of Medicine report, Retooling for an Aging America: Building the health care workforce (2008). This report states that care should require providers of various disciplines to work collaboratively in interdisciplinary teams. It also states that payment schemes need to be improved to support collaborative team care. It also emphasizes that this would mean new roles for professions as well as for patients and families. Along with improved collaborative care, this would require an improved approach to regulations and supervision that matches the collaborative nature of teams. Institutions must respond to the demand for a greater connectivity and interdependence of health disciplines. Rigid hierarchical leadership structures, professional boundaries, and a naiveté about the contributions of other disciplines must not be the core values of health care moving forward. The greatest benefits of an interdisciplinary team emerge from institutional supports that recognize the interdisciplinary team as a unit in providing patient-centered care for the frail elderly. 
The ACA has many implications for the PACE program and similar IDT-based delivery models to increase efficiency, create more patient-centered care models, and utilize the workforce in a meaningful and sustainable way. Redesigning approaches to workforce to improve care are in line with the Triple Aim and the IOM's Quality Chasm report. Restructuring the workforce and work environments is a critical consideration for more effective health care delivery. Regulation must be thoroughly considered, as current models of professional recognition and care quality are based on individual and standardized outcomes. New methods must be created to reflect patient-specific characteristics in order to contextualize quality data properly and develop team-based competencies that reflect interdependent and collaborative work by teams. The ACA provides great emphasis on team-based care in several settings; however if the institutions do not recognize how to legitimize teams in these settings, the benefits may not be realized to the extent anticipated. The ACA overall emphasizes teamwork, but it continues to default to a physician-dominated decision-making model. If teams are to meet their potential for efficiency and effectiveness, tethering them to a physician may cause problems when geriatric specialty areas are experiencing shortages of physicians (IOM, 2008). In some settings such as caring for older adults, empowering other professions in decision-making and authority would benefit organizations and teams alike.

Further legitimization of IDT-based care could occur along a number of pathways. One possible scenario would be moving away from physician-dominated decision-making. This development would involve empowering nurse practitioners, 
social workers, and other health professions with authority to facilitate team-based decision-making regarding a patient's care. Even though not considered professionals, PACE organizations should recognize the contributions of PCAs and drivers and integrate them fully into a team-based model. A second possible scenario is for regulators to reduce their regulatory footprint and work collaboratively to eliminate redundancies and reporting burdens on IDTs. This would require a relinquishing of some authority; a single oversight body would meet the needs of participant-centered teams better than several standardized regulatory processes. A third scenario would occur when the IDTs and organization create an interdependent culture along the spectrum of health care delivery including education competencies, recognition standards, and workplace structures. These three scenarios would differ in their impact on payment and regulatory structures, and continue to challenge the professional hierarchies and fee-for-service focus of care provision.

The PACE program and affiliated organizational structures have made great strides in legitimizing and increasing the feasibility of IDT care for older adults. Other models of interdisciplinary care are in the process of improving coordination and collaboration amongst health care professions and workers (Cooper, 2003; IOM, 2008). There is considerable current evidence in favor of interdisciplinary care, with many health professions school aligning their academic calendars in order to facilitate interprofessional learning. This alignment is no small feat, and the changes are slow, but there is still progress. 
Meanwhile, institutional support will continue to play an essential role in advancing collaborative workforce models in the care of older adults and other populations that require complex, interdisciplinary approaches to care. Potential new populations for this model of care may be prompted from Medicare and Medicaid funding streams, yet the cost of housing these delivery systems and the value they add has not been delineated. It is unlikely that team models will be adopted without the support of payers. The ability of the current health system to be innovative within current policy and regulative structures, much less to solve the issues with cost containment and workforce shortages in long-term care, is far from resolved. This study explored the PACE program as a model for implementing collaborative care provided by an IDT and outlined a framework for understanding the environment necessary to support practice.

\section{Assumptions and Limitations}

The fundamental assumption of this study was that the case study approach and the use of interviews would facilitate identification of contextual elements that support interdisciplinary teams in the care of older adults. A second assumption was that administrators, team members, and governing body officials would participate. A third assumption was that the use of interviews would encourage confidential communication and honest responses. The final assumption was that the cases identified would be interested in participating in the research.

Due to confidentiality concerns, the case-by-case analysis could not be done because organizations did not want to be identified. Thus, the data were aggregated to maintain confidentiality of organizational and individual identities. Some specificity of 
context was lost when specific states and communities could not be identified in the analysis of findings. There are so few PACE programs in the U.S. that any reference to particular states would identify many of the participating sites. Another limitation is that access to governing body members proved impossible in this study. The PACE program gatekeepers did not grant access to the external governance for this study. A third issue is that the balance of IDT members and administrators could have been more evenly distributed. The organizational gatekeeper, who was very protective of individual's time, limited access to IDT members.

Interviews proved an excellent means of data collection as most participants were forthcoming and made ample time available for the interview. Interviewees overall expressed interest in the topic and were very cooperative in follow-up responses. Initially, four out of five of the originally identified organizations agreed to participate. In recruiting for the fifth spot, three more organizations agreed and were added. By the time this recruitment occurred, the focus had moved away from the case-by-case analysis to an element-by-element analysis.

Major limitations in this study were inherent in the design, selection bias, limited access to resources, and a necessary single-researcher approach. No single study can do everything, or even do all of the things it tries to do as completely as one would like. Generalizability of results was problematic, which is inherent to the case-study methodology. However, the broad context of the study provides lessons for many different interest areas. Selection bias was an issue, as those that agreed to be part of the study may have similar characteristics and motivations for participating. Other 
limitations of this study include the analysis, which was conducted by one coder (the researcher), which reduces the objectivity in the coding, and a reliability calculation cannot be done. However, given that this is an individual dissertation, it is not appropriate in this context to use another coder, as a dissertation is an individual effort. The final limitation was the limited time and resources to complete this work, and the intent to complete within a specified timeframe.

\section{Recommendations for Further Research}

The findings of this study suggest a number of questions and areas for future research. Due to the interdisciplinary foundation of this study, many fields may benefit from further pursuit of issues highlighted in the findings. Gaining access to a wide audience of regulators, policy-makers, administrators, and educators of all disciplines would be instrumental in moving research forward. Collaborating with foundations to incentivize research along with organizational responses to environmental challenges will be instrumental in overcoming barriers to IDTs and elder care that have plagued health care. Some questions for future research are:

1. What is the value-added of IDT-based care for health services organizations? PACE programs have large IDTs and there are certain requirements for services provided. The reality is that the cost of service is really not known, nor is there a specific understanding of the economic value added both in terms of cost and quality by, for example, having an assessment by a dietician every 6 months. Addressing the valueadded of IDT care may go a long way in providing the value of teams in terms that are highly relevant to care provision. 
2. What level of efficacy do standardized quality measures have when the care is highly customized?

This question would begin to look at quality from a more customized perspective for elder care. PACE programs would benefit from more guidance on how to analyze the data they collect and use it through various interpretations to improve services to populations and individuals.

3. How would expanding the PCP role to include nurse practitioners as well as physicians affect PACE programs?

Each program studied expressed a desire to have greater regulatory flexibility in integrating a stronger presence for a nurse practitioner (NP) as a primary care provider (PCP) for PACE participants. CMS restrictions on PACE programs prevent the utilization of NPs as PCPs even if they have autonomous practice rights within the state. A study of this question would highlight significant issues in workforce recruitment for IDTs. As previously discussed in this chapter, physician shortages are creating issues in staffing and creation of new IDTs, flexibility in designated PCPs for IDTs would allow needed flexibility in team structure.

4. How is collaborative team function affected when supervision and accountability are provided along disciplinary lines?

There is no shortage of managers, supervisors, and other administrators in this model. IDTs would benefit from a greater understanding of how best to manage and supervise teams with such rigid regulatory requirements. The extent to which managerial levels 
understand other disciplinary expectations and their level of collaborative decisionmaking would start to illuminate this issue.

5. What supports and training can be provided to health disciplines to prepare them to weigh and assess patient needs vs. patient wants, in a patient-centered care delivery structure?

It can be difficult for IDTs to discern the fine line between keeping a participant happy and indulging them. This question would examine what, if any, learning opportunities exist for health providers to help them navigate these difficult choices, and could provide insights on most effective content areas for training

6. What are the benefits and costs to integrating entry-level staff into a professional IDT?

The value added by drivers and PCAs can support prevention-focused observations around changes in routine, favoring a recurring ache, or increased isolation, which do not currently have a consistent pathway to the IDT. Efforts to integrate all health workers in the IDT spectrum may reduce attrition in entry-level jobs, increase quality of care, and save money for the organization. No studies have been conducted to see if there is a payoff in the end to greater integration of these team members. Such a study would be a first step in addressing retention issues experienced throughout long-term care in frontline staffing.

7. What is the role of the IDT facilitator in developing interdependent team culture? 
Many studies have examined the effectiveness of teams in various settings. However, few, if any, have looked at the role of the facilitators in successful and unsuccessful teams. Most facilitators are not trained or prepared in any way, yet their role is crucial to team culture and communication flow. Moving forward, understanding the facilitator's role more in a health care context could help improve team function in various settings.

\section{Conclusions}

I don't know how the organization is going to train people to work in a team until there are more teams out there. I feel like we have to do it ourselves until we change the world (PACE Administrator, S2).

Collaborative care provided by interdisciplinary teams may address multiple ills of our current health care system. Improved care for the individual, more cost-efficiency in care delivery, and an overall improvement in population health are in alignment with the Triple Aim (Berwick, Nolan \& Whittington, 2008). Many researchers have been advocating for the use of IDTs in the care of older adults due to the complexity of care needs and the rate at which older adults utilize health care resources (Flaherty, 2004; Heinemann \& Zeiss, 2002; IOM, 2008; Siegler, 1998; Zeiss, 1996). Policy-regulative, cultural-cognitive, normative, relational, and procedural elements in an institutional environment must be in alignment to support IDT models in order to maximize the benefits to health care organizations and patients alike. As highlighted by this study, numerous obstacles make changing to a team-based care delivery model formidable, even for a well-managed health care organization.

When dealing with complex clinical issues, teams provide efficiencies to the organization and payers, as a team can distribute the burden of treatment among the staff 
and offer a more diversified and objective perspective than a solitary staff member working alone. From the patient's point of view, teams can improve access to care and other resources; they may benefit from a variety of services and perspectives from multiple disciplines. However, not all patients or patient care situations require teams. While an effective team can quickly assess a situation, decide what professionals need to be involved, and work closely together to reach a solution, assigning a team to a project may not always be the most effective or efficient use of health care resources. Some tasks can be more efficient if assigned to just one person who has the knowledge, skill, and time to do the task independently (Cashman, Reidy, Cody \& Lemay, 2004; LemieuxCharles \& McGuire, 2006; Reeves, 2010; Wise, 1974). The appropriate use of teams requires an institutional and organizational commitment to support a truly interdisciplinary approach.

In order for a widespread, consistent utilization of teams in the care of older adults, a level of institutional legitimacy is required. Merely having appropriate resources and best-practice designation is not enough. If teams are to survive and thrive within a health care institution, they will need a high level of acceptability from federal, state, and organizational stakeholders. This study shows that the PACE program demonstrates how difficult this can be to maintain. Legitimacy is not a product or service that can be owned or exchanged, but a condition that reflects an understood collaboration within the relevant cultural-cognitive, normative, and regulative frameworks. It is largely symbolic in value, but it does have great power within health care institutions to 
rearrange established modes of care delivery and create opportunities that would otherwise be impossible to fashion.

The aging process will affect every person in a different way. Physical, cognitive, or social changes are all part of the biological process of aging; however, many of us will not only be getting older but also living longer. Many individuals -- more than our current system can handle -- will require high quality, patient-centered care that will allow them to be healthy and independent as long as possible and this type of care can most effectively be provided by an interdisciplinary team. As health care delivery continues to evolve from being professionally dominated to being patient-centered, health professions will need to be interdependent to maximize their practice for the benefits of organizations and patients alike. Federal and state policies seeking to manage spiraling health care costs will require interdisciplinary teamwork and a more collaborative effort amongst the regulators. Legitimacy and support for interdisciplinary teams must come from multiple elements of the health care institutional environment, as well as legitimizing all members of the team as important providers of care to older adults. 


\section{Bibliography}

Abbott, A. D. (1988). The system of professions: An essay on the division of expert labor. Chicago, IL: University of Chicago Press.

Administration on Aging. (2012). U.S. population by age. Retrieved June 13, 2013, from http://www.aoa.gov/AoARoot/Aging_Statistics/Census_Population/census2010/Index.as $\mathrm{px}$

Allen, N. E., Foster-Fishman, P. G., \& Salem, D. A. (2002). Interagency teams: A vehicle for service delivery reform. Journal of Community Psychology, 30 (5), 475-497.

Alford, R. R., \& Friedland, R. (1985). Powers of theory: Capitalism, the state, and democracy. New York, NY: Cambridge University Press.

Andersen, B. L. (2009). What determines the behavior and performance of health professionals? Public service motivation, professional norms and/or economic incentives. International Review of Administrative Sciences, 75 (1), 79-97.

Anderson, G. (2003). Chronic care. Public Health and Policy, 3(2), 110-111.

Armstrong, D. (1985). Space and time in British general practice. Social Science \& Medicine, 20(7), 659-666.

Balas, E., Boren, S., \& Brown, G. (2000). Information technology strategies from the United States and the European Union: Transferring research to practice for health care improvement. Amsterdam: IOS Press.

Baldwin, D. C., Jr. (2007). Some historical notes on interdisciplinary and interprofessional education and practice in health care in the USA. Journal of Interprofessional Care, 21 (1), 23-37.

Bass, B. M. (1999). Two decades of research and development in transformational leadership. European Journal of Work \& Organizational Psychology, 8 (1), 9-32.

Baxter, P., \& Markle-Reid, M. (2009). An interprofessional team approach to fall prevention for older home care clients 'at risk' of falling: Health care providers share their experiences. International Journal of Integrated Care, 9 (1), 123-133.

Becker, H. S. (1961). Boys in white; student culture in medical school. Chicago: University of Chicago Press.

Bellman, L., Webster, J., \& Jeanes, A. (2011). Knowledge transfer and the integration of research, policy and practice for patient benefit. Journal of Research in Nursing, 16 (3), 254-270. 
Berwick, D. M. (2002). A user's manual for the IOM's 'Quality Chasm' report. Health Affairs, 21 (3), 80-90.

Berwick, D. M., Nolan, T. W., \& Whittington, J. (2008). The Triple Aim: Care, health, and cost. Health Affairs, 27(3), 759-769.

Bisognano, M. A., \& Kenney, C. (2012). Pursuing the Triple Aim: Seven innovators show the way to better care, better health, and lower costs. San Francisco: Jossey-Bass.

Blane, D. (2003). Health professions. In G. Scambler (Ed.), Sociology as applied to medicine. New York, NY: Saunders.

Bloom, S., Sulick, B., \& Hansen, J. C. (2011). Picking up the PACE: The Affordable Care Act can grow and expand a proven model of care. Generations, 35 (1), 53-59.

Bodenheimer, T. (1999). Disease management: Promises and pitfalls. The New England Journal of Medicine, 340 (15), 1202-5.

Booth, J., \& Hewison, A. (2002). Role overlap between occupational therapy and physiotherapy during in-patient stroke rehabilitation: An exploratory study. Journal of Interprofessional Care, 16 (1), 31-40.

Bosch, M., Dijkstra, R., Wensing, M., Van der Weijden, T., \& Grol, R. (2008). Organizational culture, team climate and diabetes care in small office-based practices. BMC Health Services Research, 8 (1), 78-94.

Boxenbaum, E., \& Jonsson, S. (2008). Isomorphism, diffusion, and decoupling. In R. Greenwood (Ed.), The SAGE handbook of organizational institutionalism (pp. 78-98). Los Angeles, CA: Sage Publishers.

Boult, C., \& Wieland, G. D. (2010). Comprehensive primary care for older patients with multiple chronic conditions: "Nobody rushes you through". Journal of the American Medical Association, 304 (17), 1936-1943.

Bragg, E., \& Hansen, J. C. (2011). A revelation of numbers: Will America's eldercare workforce be ready to care for an aging America? Generations, 34 (4), 11-19.

Bragg, E. J., Warshaw, G. A., Meganathan, K., \& Brewer, D. E. (2010). National survey of geriatric medicine fellowship programs: Comparing findings in 2006/07 and 2001/02 from the American Geriatrics Society and Association of Directors of Geriatric Academic Programs Geriatrics Workforce Policy Studies Center. Journal of the American Geriatrics Society, 58 (11), 2166-2172.

Brallier, S. A., \& Tsukuda, R. A. (2002). Organizational and team structure. In G. D. Heinemann \& A. M. Zeiss (Eds.), Team performance in health care: Assessment and development. New York: Kluwer Academic/Plenum Publishers. 
Brown, T. B. (1982). A historical view of health care teams. In G. J. Agich (Ed.), Responsibility in health care. Boston, MA: Kluwer Boston.

Bruce, N. (1980). Teamwork for preventive care. New York, NY: Research Studies Press. Butler, R. N. (1975). Why survive?: Being old in America. New York, NY: Harper \& Row.

Cabot, R. C. (1915). Social service and the art of healing. Washington, DC: National Association of Social Workers.

Cashman, S., Reidy, P., Cody, K., \& Lemay, C. (2004). Developing and measuring progress toward collaborative, integrated, interdisciplinary health care teams. Journal of Interprofessional Care, 18 (2), 183-196.

Center on Budget and Policy Priorities. (2012). Where do our federal tax dollars go? Policy Basics, 4(1), 4. Retrieved from http://www.cbpp.org/cms/index.cfm?fa=view\&id=1258

Centers for Medicare and Medicaid Services (CMS). (2006). Medicare and Medicaid programs; programs of all-inclusive care for the elderly (PACE); program revisions. Final rule. Federal register, 71(236), 71243-71337.

Centers for Medicare and Medicaid Services. (2010). Medicare hospice benefits. Retrieved May 20, 2012, from http://purl.fdlp.gov/GPO/gpo2012

Centers for Medicare and Medicaid Services. (2011). Prospective Payments Systems. Retrieved March 10, 2011, from https://www.cms.gov/ProspMedicareFeeSvcPmtGen/

Chafetz, P. K., West, H., \& Ebbs, E. (1987). Overcoming obstacles to cooperation in interdisciplinary long term care teams. Journal of Gerontological Social Work, 11 (3), 131-140.

Chatterji, P., Burstein, N., Kidder, D., White, A. J., \& Abt Associates. (1998). Evaluation of the Program of All-Inclusive Care for the Elderly (PACE) demonstration: The impact of PACE on participant outcomes. Cambridge, Mass: Abt Associates.

Cherkasky, M. (1949). The Montefiore Hospital Home Care Program. American Journal of Public Health, 39 (2), 163-166.

Conrad, P., \& Kern, R. (2005). The sociology of health and illness: Critical perspectives. New York: St. Martin's Press.

Cook, R. G. (2003). 7 formulas for team success: Seven straightforward formulas to clarify group dynamics. Industrial Management, 45 (6), 21-28. 
Coogle, C., Parham, I., Cotter, J., Welleford, E., \& Netting, F. (2005). A professional development program in geriatric interdisciplinary teamwork: Implications for managed care and quality of care. The Journal of Applied Gerontology, 24 (2), 142-159.

Cooper, B. S., \& Fishman, E. (2003). The interdisciplinary team in the management of chronic conditions: Has its time come? Baltimore, MD: Partnership for Solutions.

Counsell, S. R., Callahan, C. M., Tu, W., Stump, T. E., \& Arling, G. W. (2009). Cost Analysis of the Geriatric Resources for Assessment and Care of Elders Care Management Intervention. Journal of the American Geriatrics Society, 57 (8), 1420-1426.

Crimmins, E. M. (2004). Trends in the health of the elderly. Annual Review of Public Health, 25 (1), 79-98.

Crinson, I. (2008). The health professions. In G. Scambler (Ed.), Sociology as applied to medicine. New York: Saunders/Elsevier.

Currie, G., Finn, R., \& Martin, G. (2008). Accounting for the 'dark side' of new organizational forms: The case of healthcare professionals. Human Relations, 61 (4), 539-564.

D'Andrade, R. G. (1984). Cultural meaning systems In R. A. Shweder \& R. A. LeVine (Eds.), Culture theory: Essays on mind, self, and emotion. Cambridge, NY: Cambridge University Press.

David, A. M. (2010). The social psychology of status leveling in organizational contexts. International Journal of Organizational Analysis, 18 (1), 76-104.

Davis, K., \& Collins, S. R. (2005). Medicare at forty. Health Care Financing Review, 27 (2), 2005-2006.

Deephouse, D., \& Suchman, M. (2008). Legitimacy in organizational institutionalism. In R. Greenwood (Ed.), The SAGE handbook of organizational institutionalism (pp. 49-77). Los Angeles, CA: Sage Publishers.

Dellefield, M. E. (2006). Interdisciplinary care planning and the written care plan in nursing homes: A critical review. The Gerontologist, 46 (1), 128-133.

Delva, D., Jamieson, M., \& Lemieux, M. (2008). Team effectiveness in academic primary health care teams. Journal of Interprofessional Care, 22 (6), 598-611.

Dieleman, S., Farris, K., Feeny, D., Johnson, J., Tsuyuki, R., \& Brilliant, S. (2004). Primary health care teams: Team members' perceptions of the collaborative process. Journal of Interprofessional Care, 18 (1), 75-78.

DiMaggio, P. J., \& Powell, W. W. (1983). The iron cage revisited: Institutional isomorphism and collective rationality in organizational fields. American Sociological Review, 48 (2), 147-160. 
DiPalma, C. (2004). Power at work: Navigating hierarchies, teamwork and webs. Journal of Medical Humanities, 25 (4), 291-308.

Douglas, T. (2000). Basic groupwork. New York, NY: Routledge.

Drinka, T. J. K., \& Clark, P. G. (2000). Health care teamwork: Interdisciplinary practice and teaching. Westport, CT: Auburn House.

Ducanis, A. J., \& Golin, A. K. (1979). The interdisciplinary health care team: A handbook. Germantown, Md.: Aspen Systems Corp.

Durkheim, É. (1976). The elementary forms of the religious life. London, England: Allen and Unwin.

Eng, C., Pedulla, J., Eleazer, G. P., McCann, R., \& Fox, N. (1997). Program of Allinclusive Care for the Elderly (PACE): An innovative model of integrated geriatric care and financing. Journal of the American Geriatrics Society. 45 (2), 223.

Engestrom, Y. (1999). When the center does not hold. In S. Chaiklin, M. Hedegaard \& U. J. Jensen (Eds.), Activity theory and social practice: Cultural-historical approaches. Oakville, CN: Aarhus University Press.

Evetts, J. (2006). Short note: The sociology of professional groups. Current Sociology, 54 (1), 133-143.

Farrell, M., Schmitt, M., \& Heinemann, G. (2001). Informal roles and the stages of interdisciplinary team development. Journal of Interprofessional Care, 15 (3), 281-295.

Ferlie, E. (1996). The new public management in action. New York, NY: Oxford University Press.

Fiorelli, J. S. (1988). Power in work groups: Team member's perspectives. Human Relations Human Relations, 41 (1), 1-12.

Flaherty, E. (2004). Frail older patient care by interdisciplinary teams. Gerontology \& Geriatrics Education, 24 (2), 51-62.

Foucault, M. (1978). The history of sexuality. New York, NY: Pantheon Books.

Friedland, R., \& Alfred, R. (1991). Bringing society back in: Symbols, practice and institutional contradictions. In W. W. Powell \& P. DiMaggio (Eds.). The new institutionalism in organizational analysis. Chicago, IL: University of Chicago Press.

Friedson, E. (2001). Professionalism: The third logic. Chicago: University of Chicago Press.

Friedson, E. (1986). Professional powers: A study of the institutionalization of formal knowledge. Chicago: University of Chicago Press. 
Friedson, E. (1988). Profession of medicine: A study of the sociology of applied knowledge. Chicago: University of Chicago Press.

Friedman, M. (1962). Capitalism and freedom. Chicago: University of Chicago Press.

Gardezi, F., Lingard, L., Espin, S., Whyte, S., Orser, B., \& Baker, G. R. (2009). Silence, power and communication in the operating room. Journal of Advanced Nursing, 65 (7), 1390-1399.

Gawande, A. (2007). The way we age now: Can medicine serve an aging population? The New Yorker, 83 (11), 50-59.

Gibbon, B. (1999). An investigation of interprofessional collaboration in stroke rehabilitation team conferences. Journal of Clinical Nursing, 8 (3), 246-252.

Glaser, B. G., \& Strauss, A. L. (1967). The discovery of grounded theory: Strategies for qualitative research. Hawthorne, NY: Aldine de Grunter Press.

Goodson, J. D. (2010). Patient Protection and Affordable Care Act: Promise and peril for primary care. Annals of Internal Medicine, 152 (11), 742-744.

Grabowski, D. (2006). The cost-effectiveness of noninstitutional long-term care services: Review and synthesis of the most recent evidence. Medical Care Research and Review, 63 (1), 3-28.

Greenwood, R. (2008). The Sage handbook of organizational institutionalism. Los Angeles, CA: Sage Publishers.

Greenwood, R., Suddaby, R., \& Hinings, C. R. (2002). Theorizing change: The role of professional associations in the transformation of institutionalized fields. Academy of Management Journal, 45 (1), 58-80.

Grinspun, D. (2007). Healthy workplaces: The case for shared clinical decision making and increased full-time employment. Health Care Papers, 7 (1), 85-91.

Gross, D., Temkin-Greener, H., Kunitz, S. \& Mukamel, D. (2004). The growing pains of integrated health care for the elderly: Lessons from the expansion of PACE. Milbank Quarterly, 82 (2), 257-282.

Gobi, E., \& Lincoln, Y. S. (2005). Competing paradigms in qualitative research. In N. K. Denzin \& Y. S. Lincoln (Eds.), The Sage handbook of qualitative research (pp. 105-117). Thousand Oaks, CA: Sage Publications.

Handy, C. B. (1999). Understanding organizations. New York, NY: Penguin.

Hannan, M., \& Freeman, J. (1984). Structural inertia and organizational change. American Sociological Review, 49 (2), 149-164. 
Hardy, C., \& Maguire, S. (2008). Institutional entrepreneurship. In R. Greenwood (Ed.), The Sage handbook of organizational institutionalism. Los Angeles, CA: Sage

Publishers.

Harrington, C., Carrillo, H., Wellin, V., Norwood, F., \& Miller, N. (2001). Access of target groups to 1915 (c) medicaid home and community based waiver services. Home Health Care Services Quarterly, 20 (2), 61-80.

Harrington, C., \& Estes, C. L. (2008). Health policy: Crisis and reform in the U.S. health care delivery system. Sudbury, Mass: Jones and Bartlett.

Hartman, M., Martin, A., McDonnell, P., \& Catlin, A. (2009). National health spending in 2007: Slower drug spending contributes to lowest rate of overall growth since 1998. Health Affairs, 28 (1), 246-261.

Healey, A. N., Undre, S., \& Vincent, C. A. (2006). Defining the technical skills of teamwork in surgery. Quality \& Safety in Health Care, 15 (4), 231-234.

Heath, H., \& Cowley, S. (2004). Developing a grounded theory approach: A comparison of Glaser and Strauss. International Journal of Nursing Studies, 41 (2), 141-150.

Heinemann, G. D., \& Zeiss, A. M. (2002). Team performance in health care: Assessment and development. New York, NY: Kluwer Academic Publishers.

Herzlinger, R. E. (2004). Consumer-driven health care: Implications for providers, payers, and policymakers. San Francisco, CA: Jossey-Bass.

Higginson, I. J., Finlay, I. G., Goodwin, D. M., Hood, K., Edwards, A. G. K., Cook, A., \& Normand, C. E. (2003). Is there evidence that palliative care teams alter end-of-life experiences of patients and their caregivers? Journal of Pain and Symptom Management, $25(2), 150$.

Hinings, C. (2008). Organizational institutionalism and sociology. In R. Greenwood (Ed.), The Sage handbook of organizational institutionalism. Los Angeles, CA: Sage Publishers.

Hirth, V., Baskins, J., \& Dever-Bumba, M. (2009). Program of All-Inclusive Care (PACE): Past, present, and future. Journal of the American Medical Directors Association, 10 (3), 155-160.

Hugman, R. (1991). Power in caring professions. Basingstoke, England: Macmillan. Inouye, S. K., Bogardus, S. T., Jr., Baker, D. I., Leo-Summers, L., \& Cooney, L. M., Jr. (2000). The Hospital Elder Life Program: A model of care to prevent cognitive and functional decline in older hospitalized patients. Hospital Elder Life Program. Journal of the American Geriatrics Society, 48 (12), 1697-1706. 
Institute of Medicine. (1996). 2020 vision: Health in the 21st century. Washington, D.C.: National Academy Press.

Institute of Medicine. (2001). Crossing the quality chasm: A new health system for the 21 st century. Washington, D.C.: National Academy Press.

Institute of Medicine. (2008). Retooling for an aging America: Building the health care workforce. Washington, D.C.: National Academies Press.

Institute of Medicine. (2009). Informing the future critical issues in health. Washington, D.C.: Institute of Medicine of the National Academies.

Jacobsen, L. A., Kent, M., Lee, M., \& Mather, M. (2011). America's aging population. Population Bulletin. 66 (1). Access date: March 16, 2011. Retrieved from www.prb.org/pdf11/aging-in-america.pdf

Jaques, D. (2002). Learning in groups: A handbook for improving group work, 3rd edition. Peace Research, 3 (1), 3-152.

Jelphs, K., \& Dickinson, H. (2008). Working in teams. Bristol, England: Policy Press.

Joint Commission on Accreditation of Healthcare Organizations. (2000). Improving the care experience. Oakbrook Terrace, IL: Joint Commission on Accreditation of Healthcare Organizations.

Joint Commission on Accreditation of Healthcare Organizations. (2006). A pocket guide to using performance improvement tools in health care settings. Oakbrook Terrace, IL: Joint Commission Resources.

Joint Commission on Accreditation of Healthcare Organizations. (2011a). Ambulatory Care Accreditation Standards by Focus Area. Washington, D.C.: The Joint Commission.

Joint Commission on Accreditation of Healthcare Organizations. (2011b). Approved Standards for the Joint Commission Primary Care Medical Home Option, Ambulatory Care Accreditation Program (Vol. HR.03.01.01). Washington, D.C.: Joint Commission on Accreditation of Healthcare Organizations.

Joint Commission on Accreditation of Healthcare Organizations. (2011c). Long-Term Care Accreditation Standards. Washington, D.C.: The Joint Commission.

Kaiser Family Foundation. (2012). Kaiser State Health Facts [Website]. Retrieved August 10, 2012 from http://www.statehealthfacts.org/index.jsp

Kane, R. L., \& Blewett, L. A. (1993). Quality assurance for a program of comprehensive care for older persons. Health Care Financing Review, 14 (4), 89-110.

Kane, R. L., Illston, L. H., \& Miller, N. A. (1992). Qualitative analysis of the Program of All-inclusive Care for the Elderly (PACE). The Gerontologist, 32 (6), 771-780. 
Katzenbach, J. R., \& Smith, D. K. (1994). The wisdom of teams: Creating the highperformance organization. New York, NY: Harper Business.

Kaye, H. S., Chapman, S., Newcomer, R. J., \& Harrington, C. (2006). The personal assistance workforce: Trends in supply and demand. Health Affairs, 25 (4), 1113-1120.

Kitchener, M., \& Exworthy, M. (2008). Models of medical work control: A theory elaboration from English general practice. In McKee, L., Ferlie, E., \& Hyde, P. (Eds.), Organizing and re-organizing: Power and change in health-care organizations (pp. 209-223). Palgrave Macmillan.

Kohn, L. T., Corrigan, J., \& Donaldson, M. S. (2000). To err is human: Building a safer health system. Washington, D.C.: National Academy Press.

Lammers, J. C., \& Barbour, J. B. (August 01, 2006). An institutional theory of organizational communication. Communication Theory, 16 (3), 356-377.

Leicht, K., Walter, T., Sainsaulieu, I., \& Davies, S. (2009). New public management and new professionalism across nations and contexts. Current Sociology, 57 (4), 581-605.

Lemieux-Charles, L., \& McGuire, W. (2006). What do we know about health care team effectiveness? A review of the literature. Medical Care Research and Review, 63 (3), 263-300.

Levay, C., \& Waks, C. (2009). Professions and the pursuit of transparency in healthcare: Two cases of soft autonomy. Organization Studies, 30 (5), 509-527.

Lewin, S., \& Reeves, S. (2011). Enacting 'team' and 'teamwork': Using Goffman's theory of impression management to illuminate interprofessional practice on hospital wards. Social Science \& Medicine, 72 (10), 1595-1602.

Liao, S., \& Ackermann, R. (2008). Interdisciplinary end-of-life care in nursing homes. Clinical Gerontologist, 31 (4), 83-96.

Light, D. W. (1991). Professionalism as a countervailing power. Journal of Health Politics, Policy and Law, 16 (3), 499-506.

Lorenz, K., \& Agency for Healthcare Research Quality. (2004). End-of-life care and outcomes: Summary. Rockville, MD: Agency for Healthcare Research and Quality.

Lounsbury, M., \& Crumley, E. (2007). New practice creation: An institutional perspective on innovation. Organization Studies, 28 (7), 993-1012.

Lynch, M., Hernandez, M., \& Estes, C. (2008). PACE: Has it changed the chronic care paradigm? Social Work in Public Health, 23 (4), 3-24.

Macdonald, K. M. (1995). The sociology of the professions. Thousand Oaks, CA: Sage Publishers. 
Mackay, S. (2004). The role perception questionnaire (RPQ): A tool for assessing undergraduate students' perceptions of the role of other professions. Journal of Interprofessional Care, 18 (3), 289-302.

Mahmood-Yousuf, K., Munday, D., King, N., \& Dale, J. (2008). Interprofessional relationships and communication in primary palliative care: Impact of the Gold Standards Framework. The British Journal of General Practice., 58 (549), 256-263.

Marshall, C., \& Rossman, G. B. (1999). Designing qualitative research. Thousand Oaks, CA: Sage.

Martin, V., \& Rogers, A. M. (2004). Leading interprofessional teams in health and social care. New York, NY: Rutledge.

Medicare Payment Advisory Commission. (2010). Healthcare spending and the Medicare program. Date accessed: April 4, 2011 Available from http://purl.access.gpo.gov/GPO/LPS79103

Merton, R. K. (1968). Social theory and social structure. New York, NY: Free Press.

Meyer, J. W., \& Rowan, B. (1977). Institutionalized organizations: Formal structure as myth and ceremony. American Journal of Sociology, 83(2).

Meyer, J. W., \& Scott, W. R. (1992). Organizational environments: Ritual and rationality. Newbury Park, CA: Sage Publications.

Miller, E., Booth, M., \& Mor, V. (2008). Assessing experts' views of the future of longterm care. Research on Aging, 30(4), 450-473.

Mion, L., Odegard, P. S., Resnick, B., \& Segal-Galan, F. (2006). Interdisciplinary care for older adults with complex needs: American Geriatrics Society position statement. Journal of the American Geriatrics Society, 54 (5), 849-852.

Mitchell, R., Parker, V., Giles, M., \& White, N. (2010). Review: Toward realizing the potential of diversity in composition of interprofessional health care teams. Medical Care Research and Review, 67 (1), 3-26.

Morrison, R. S., \& Meier, D. E. (2004). Palliative care. The New England Journal of Medicine, 350 (25), 2582.

Mukamel, D. B., Temkin-Greener, H., Delavan, R., Peterson, D. R., Gross, D., Kunitz, S., \& Williams, T. F. (2006). Team performance and risk-adjusted health outcomes in the Program of All-Inclusive Care for the Elderly (PACE). Gerontologist, 46 (2), 227-237.

National PACE Association. (2004). State resources. Retrieved July 15, 2013, fromhttp://www.npaonline.org/website/article.asp?id=731\&title=State_Resources 
National PACE Association. (2013). What is PACE? Retrieved May 25, 2013, from http://www.npaonline.org/website/article.asp?id=12

Newcomer, R., Harrington, C., \& Friedlob, A. (1990). Social Health Maintenance Organizations: Assessing their initial experience. Health Services Research, 25 (3), 425 54.

Ng, T., Harrington, C., \& Kitchener, M. (2010). Medicare and Medicaid in long-term care. Health Affairs, 29 (1), 22-28.

North, D. C. (1990). Institutions, institutional change, and economic performance. New York, NY: Cambridge University Press.

Oliver, C. (1992). The antecedents of deinstitutionalization. Organization Studies, 13 (4), 563-588.

Onyett, S., \& Campling, J. (2003). Teamworking in mental health. New York, N.Y.: Palgrave Macmillan.

Owen-Smith, J., \& Powell, W. (2008). Networks and institutions. In R. Greenwood (Ed.), The SAGE handbook of organizational institutionalism (pp. 596-622). Los Angeles, CA: Sage Publishers.

Palley, H. A. (2003). Long-term care policy for older Americans: Building a continuum of care. Journal of Health \& Social Policy, 16 (3), 7-18.

Petigara, T. \& Anderson, G. (2009) Program of All-Inclusive Care for the Elderly. Health Policy Monitor. Retrieved July 22, 2013 from http://www.hpm.org/survey/us/b13/2

2009 Pfeffer, J., \& Salancik, G. R. (1978). The external control of organizations: A resource dependence perspective. New York: Harper \& Row.

Phillips, N., \& Malhotra, N. (2008). Taking social construction seriously: Extending discursive approach in institutional theory. In R. Greenwood (Ed.), The Sage handbook of organizational institutionalism (pp. 702-720). Los Angeles, CA: Sage Publishers.

Pleis, J. R., \& Lethbridge-Cejku, M. (2007). Summary health statistics for U.S. adults: National Health Interview Survey 2006 Vital and health statistics. Series 10, Data from the National Health Survey (pp. 1-153). Available from:

www.cdc.gov/nchs/data/series/sr_10/sr10_252.pdf

Pleis, J. R., Ward, B. W., \& Lucas, J. W. (2010). Summary health statistics for U.S. adults: National Health Interview Survey, 2009. Hyattsville, MD: U.S. Dept. of Health and Human Services, Centers for Disease Control and Prevention, National Center for Health Statistics. Available from: www.cdc.gov/nchs/data/series/sr_10/sr10_252.pdf

Proudfoot, J. G., Bubner, T., Amoroso, C., Swan, E., Holton, C., Winstanley, J. \& Harris, M. F. (2009). Chronic care team profile: A brief tool to measure the structure and 
function of chronic care teams in general practice. Journal of Evaluation in Clinical Practice, 15 (4), 692-698.

Pryor, J. (2008). A nursing perspective on the relationship between nursing and allied health in inpatient rehabilitation. Disability and Rehabilitation, 30 (4), 314-322.

QSR International Pty Ltd. (2012). NVIVO: Qualitative analysis software (Version 10) [Software]. Available from: http://www.qsrinternational.com/products_nvivo.aspx.

Rafferty, A. M., Ball, J., \& Aiken, L. H. (2001). Are teamwork and professional autonomy compatible, and do they result in improved hospital care? Quality in Health Care, 10 (1), 32-37.

Reeves, S. (2010). Interprofessional teamwork for health and social care. Oxford: WileyBlackwell.

Reeves, S., Freeth, D., Glen, S., Leiba, T., Berridge, E. J., \& Herzberg, J. (2006). Delivering practice-based interprofessional education to community mental health teams: Understanding some key lessons. Nurse Education in Practice, 6 (5), 246-253.

Reinhard, S., Houser, A., Kassner, E., \& Mollica, R. (2011). Raising Expectations: A State Scorecard on Long-Term Services and Supports for Older Adults, People With Physical Disabilities, and Family Caregivers. Retrieved May 25, 2012 from http://www.longtermscorecard.org/ /media/Files/Scorecard\%20site/Report/AARP_Reinh ard_Realizing_Exp_LTSS_Scorecard_REPORT_WEB_v4.pdf

Rogers, D. (1932). Teamwork within the hospital. The American Journal of Nursing, 32 (6), 657-659.

Rubin, F. H., Williams, J. T., Lescisin, D. A., Mook, W. J., Hassan, S., \& Inouye, S. K. (2006). Replicating the Hospital Elder Life Program in a community hospital and demonstrating effectiveness using quality improvement methodology. Journal of the American Geriatrics Society, 54 (6), 969-974.

Rudolph, N. V., \& Lubitz, J. (January 01, 1999). Capitated payment approaches for Medicaid-financed long-term care services. Health Care Financing Review, 21 (1), 5164.

Rutherford, J., \& McArthur, M. (2004). A qualitative account of the factors affecting team-learning in primary care. Education for Primary Care, 15 (3), 352-360.

Ryan, D. P. (1996). A history of teamwork in mental health and its implications for teamwork training and education in gerontology. Educational Gerontology, 22 (5), 411431.

Schmitt, M. (1994). USA: Focus on interprofessional practice, education, and research. Journal of Interprofessional Care, 8 (1), 9-18. 
Schulz, J. H. (2001). The economics of aging. Westport, CN: Auburn House.

Scott, W. R. (2000). Institutional change and healthcare organizations: From professional dominance to managed care. Chicago, IL: University of Chicago Press.

Scott, W. R. (2003). Organizations: Rational, natural, and open systems (5th ed.). Upper Saddle River, NJ: Prentice Hall.

Scott, W. R. (2008a). Institutions and organizations: Interests and ideas. Thousand Oaks: Sage.

Scott, W. (2008b). Lords of the Dance: Professionals as Institutional Agents. Organization Studies, 29 (2), 219-238.

Scott, W. R., \& Davis, G. F. (2007). Organizations and organizing: Rational, natural, and open system perspectives. Upper Saddle River, NJ: Pearson Prentice Hall.

Scott, W. R., \& Meyer, J. W. (1994). Institutional environments and organizations: Structural complexity and individualism. Thousand Oaks, CA: SAGE Publications.

Seago, J. A., \& Ash, M. (2002). Registered nurse unions and patient outcomes. The Journal of nursing administration, 32 (3), 143-151.

Sexton, J., Thomas, E., \& Helmreich, R. (2000). Error, stress, and teamwork in medicine and aviation: Cross sectional surveys. British Medical Journal, 320 (7237), 745-749.

Shortell, S. M. (1985). High-performing healthcare organizations: Guidelines for the pursuit of excellence. Hospital \& Health Services Administration, 30 (4), 7-35.

Siegler, E. L. (1998). Geriatric interdisciplinary team training. New York, NY: Springer Publishing Company.

Silver, G. A. (1958). Beyond general practice: The health team. The Yale Journal of Biology and Medicine, 31 (1), 29-39.

Skocpol, T., Evans, P. B., \& Rueschemeyer, D. (1985). Bringing the state back in. Cambridge, NY: Cambridge University Press.

Social Security Administration. (2010). Status of the Social Security and Medicare programs: A summary of the 2010 reports. Retrieved August 5, 2010, from http://www.ssa.gov/oact/trsum/index.html.

Stake, R. E. (2006). Multiple case study analysis. New York: Guilford Press.

Stark, S., Stronach, I., \& Warne, T. (2002). Teamwork in mental health: Rhetoric and reality. Journal of Psychiatric \& Mental Health Nursing, 9 (4), 411-418.

Starr, P. (1982). The social transformation of American medicine. New York, NY: Basic Books. 
Stock, R. D., Reece, D., \& Cesario, L. (2004). Models and systems of geriatric care: Developing a comprehensive interdisciplinary senior healthcare practice. Journal of the American Geriatrics Society, 52 (12), 2128-2133.

Stone, R. I. (2004). The direct care worker: The third rail of home care policy. Annual Review of Public Health, 25 (1), 521-537.

Stone, R.I. (2000). Long-term care for the elderly with disabilities: Current policy, emerging trends, and implications for the twenty-first century. New York, NY: Milbank Memorial Fund.

Suchman, M. C. (1995). Managing legitimacy: Strategic and institutional approaches. Academy of Management Review, 20 (3), 571-610.

Taylor, D. H. (2009). The effect of hospice on Medicare and informal care costs: The U.S. experience. Journal of Pain and Symptom Management, 38 (1), 110-114.

Temkin-Greener, H. (2006). Advance care planning in a frail older population: Patient versus program influences. Abstracts in Social Gerontology, 49 (1), 8-10.

Thompson, F. J., \& Burke, C. (2010). Federalism by waiver: Medicaid and the transformation of long-term care. Publius, 39 (1), 22.

Thornton, P., \& Ocasio, W. (2008). Institutional logics. In R. Greenwood (Ed.), The Sage handbook of organizational institutionalism. (pp. 99-129). Los Angeles, CA: Sage

Publishers.

Tolbert, P., \& Zucker, L. (1996). Institutionalization of institutional theory. In S. Clegg, C. Hardy \& W. R. Nord (Eds.), Handbook of organization studies. Thousand Oaks, CA: Sage Publications.

Tsukuda, R. A. W. (2005). Comprehensive analysis of health care team literature using information synthesis. (Doctoral Dissertation). Seattle, WA: Seattle University.

United States Board of Trustees of the Federal Hospital Insurance Trust. (2011). The 2011 annual report of the Boards of Trustees of the Federal Hospital Insurance and Federal Supplementary Medical Insurance Trust. Washington, DC: U.S. Government Printing Office.

United States Census Bureau. (2012). State and County QuickFacts [Website]. Retrieved August 17, 2012, from http://quickfacts.census.gov/qfd/index.html

Vogt, K. (2006). Productivity versus case management in hospice. Home Health Care Management \& Practice, 18 (6), 467-478.

Ward, D. (1991). Who's going to do the dirty work? Essay review. Journal of Health Politics, Policy and Law, 16 (4), 823-830. 
Warren, R. L. (1967). The interorganizational field as a focus for investigation. Administrative Science Quarterly, 12 (3), 396-419.

Warshaw, G. A., \& Bragg, E. J. (2003). The training of geriatricians in the United States: Three decades of progress. Journal of the American Geriatrics Society, 51 (7 suppl). S338-S345.

Wenzel, R. P. \& Rohrer, J. E. (1994). The iron triangle of health care reform. Clinical Performance and Quality Health Care, 2 (1), 7-9.

West, M. A., \& Markiewicz, L. (2004). Building team-based working: A practical guide to organizational transformation. Malden, MA: Blackwell.

Williams, T. F. (1986). Geriatrics: The fruition of the clinician reconsidered. The Gerontologist, 26 (4), 345-349.

Wilson-Barnett, J. (1989). Limited autonomy and partnership: Professional relationships in health care. Journal of Medical Ethics, 15 (1), 12-16.

Wise, H. F. (1974). Making health teams work. Cambridge, MA: Ballinger Publishing Company.

Wooten, M., \& Hoffman, A. (2008). Organizational fields: Past, present, and future. In R. Greenwood (Ed.), The Sage handbook of organizational institutionalism (pp. 130-148). Los Angeles, CA: Sage Publishers.

Wunderlich, G. S., \& Kohler, P. O. (2001). Improving the quality of long-term care. Washington, D.C.: National Academy Press.

Xyrichis, A., \& Lowton, K. (2008). What fosters or prevents interprofessional teamworking in primary and community care? A literature review. International Journal of Nursing Studies, 45 (1), 140-153.

Yeatts, D. E., \& Hyten, C. (1998). High-performing self-managed work teams: A comparison of theory to practice. Thousand Oaks, CA: Sage Publications.

Yin, R. K. (2009). Case study research: Design and methods. Los Angeles, CA: Sage Publications.

Zeiss, A. M., \& Steffen, A. M. (1996). Interdisciplinary health care teams: The basic unit of geriatric care. In L. L. Carstensen, B. A. Edelstein \& L. Dornbrand (Eds.), The practical handbook of clinical gerontology. (pp. 423-450): Thousand Oaks, CA: Sage Publications.

Zucker, L. G. (1987). Institutional theories of organization. Annual Review of Sociology, 13 (1), 443-464. 
Zucker, L. G. (1983). Organizations as institutions. In S. B. Bacharach (Ed.), Research in the sociology of organizations (pp. 1-42). Greenwich, CN: JAI Press.

Zucker, L. G. (1977). The role of institutionalization in cultural persistence. American Sociological Review, 42 (5), 726-743. 


\section{Appendix A: Human Subjects Research Review Committee (HSRRC) Application}

Application for HSRRC approval was submitted October 8, 2012 to Portland

State University’s HRSSC. Approval was received October 21, 2012 (\#122337).

Project Title: Interdisciplinary Teams in the Care of Older Adults: The institutional context that supports and legitimizes practice

Prospectus: The purpose of this study is to examine the relationship between elements of institutional context and the utilization of interdisciplinary teams, and explores the extent to which institutional decision-makers consider team-based models in policy and procedure changes in delivery of care for older adults. This research will use a multiple case study design and data will be collected through key informant interviews. The results of this study will clarify how an organization can integrate team-based care and what it means for stakeholders of the health care institution to support team-based care. This is a part of a dissertation in the Public Affairs and Policy PhD program in the Hatfield School. It was approved at a colloquium on June 14, 2012. As a dissertation, this work will be conducted independently, while consulting with the dissertation committee as necessary.

The research question to be investigated is: What elements of institutional context support the use of interdisciplinary teams in the care of older adults? The focus of this research is to examine the institutional context of five distinct institutions that have implemented the Program for All-Inclusive Care for the Elderly (PACE) in order to care for their frail elderly population. PACE is a jointly funded Medicare/Medicaid car 
provider that, since 1997, has required care be provided by an interdisciplinary team. This study will focus on an organizational level of analysis and will collect data using interviews and document review. Subjects for this research will be leadership and staff of the organizations. No information will be required about patients and their families. Patients and their families will not be asked to provide an interview or other documentation.

Type of Review: I am requesting an expedited review because risk is minimal to human subjects. Data will be collected through digital voice recording, and a standardized interview protocol will be used to collected data.

Subject Recruitment: PACE organizations are required to provide services via interdisciplinary team, so these programs were selected to create the cohort for this study. There are 90 PACE programs certified by CMS nationwide; in order to select the most appropriate sites, eight criteria were developed from the literature and were used to identify the sites that would provide maximum information about variations in context. These criteria include: PACE certification, years of operation, organizational form, overall long-term services and supports system performance, percentage of the local population over the age of 65 , percentage of the state population over 65 who belong to a minority group, those over 65 years in the state that are living in poverty, and the state position on the Affordable Care Act. Using a theoretically informed selection hierarchy, five sites were selected from the list of 90 , with five more identified as back-ups. 
Each program will be invited to participate in the study through its top-level manager. Depending on organizational form, other permissions to conduct research may be required, prior to conducting interviews. These procedures will be completed before recruiting individuals for interviews. Key informants will then be selected based on management level; one individual will be selected per level to provide a different perspective on the context. Interviews will utilize a semi-structured interview protocol that will inquire about the institutional structures relevant to interdisciplinary team support and legitimization. Five interviews will be completed per site. Invitations to participate will be extended first to the Director (Executive, Medical or Program) of the PACE program, then to other members of the staff as described below, totaling five interviews per site. Interview invitations will be extended to the following individuals:

1. 1 Executive Director/Program Director/Medical Director

2. 1 Team Members

3. 1-2 other interviews will be recruited with referrals by key informants that have already been interviewed. These may include the quality assurance and improvement representative, a member of the participant advisory committee, former program or medical directors, or team leaders.

The interviews are expected to take at least 90 minutes each for the Director and Team Member. The interviews will be administered in person, over the phone or via Skype.

Informed Consent: All subjects in this study will be over the age of 18, thus one level of consent is required. All key informants will be informed that their participation is 
voluntary and that their responses will be kept confidential and reported in aggregate only with identities concealed. A consent form will be provided in advance of each interview for the informant to review and sign. Each informant will receive a copy of the signed consent form to keep for his or her records. All informants will have multiple opportunities to decline participation.

First-Person Scenario: "I received an email from Ms. Foucek Tresidder at Portland State University. As a part of her dissertation, she requested my participation in an interview to talk about how the interdisciplinary team is developed within our Program for the All-Inclusive Care of the Elderly (PACE) program and how it is supported. She stated that she talked to our $\mathrm{CEO}$ and received permission to contact PACE staff for interviews. She indicated that a paper letter would follow with a further introduction and a copy of the consent form informing me of my rights as a participant in the research. She also offered to send me the questions I would be asked, which I appreciated and made me feel more comfortable with the interview. I responded to her that I would be happy to grant her an interview. A few days later, I received a call from Ms. Foucek Tresidder, and we set up an appointment to talk via Skype [in-person or phone]. She requested the appropriate contact information and called at the appointed time. We talked for nearly 90 minutes and I answered her questions. I talked about our program and organization, my role, the interdisciplinary team function and support, and ongoing quality improvement activities. We talked about how external and internal factors may affect team functioning and how the PACE program has changed the way the larger 
organization serves clients. She thanked me for talking to her and asked if she may contact me for further clarification if necessary and I agreed."

Potential Risks and Safeguards: There are no physical or psychological risks to the interviewees. There will be a cost in terms of time to those participating. The interviews are expected to take at least 90 minutes each for the Director and Team Member protocols and 60 minutes for the Governing Body Member protocol. There may be some follow-up contacts to update, clarify points, or obtain further information; these time demands should be minimal and will be conducted via email or phone. I will code all interviews; the codes will be used in notes as well as in transcription. The use of the data is for this dissertation and subsequent journal articles or conference papers. Only the committee members and the researcher will know the identities of the research participants. Prior to each interview, interviewees will be asked to review and sign a consent form. My committee members will not hear the recordings or see the raw transcripts in order to preserve the confidentiality of responses.

Potential Benefits: There are a few benefits to the research sites and individuals from their participation in this research. Upon completion of the research, the PACE program will be invited to receive a presentation and/or written report about their site. Sites will be able to withdraw from the study at any time. If an individual withdraws or declines to be interviewed, then another staff/leadership informant will be identified. If an entire site withdraws or declines participation, then the selection hierarchy will be used to identify another organization with similar characteristics and it will be recruited. If time remaining becomes an issue, the dissertation committee will be consulted as to the 
necessity for further recruitment of subjects. Participation in this study will contribute to the growing body of literature relevant to interdisciplinary teamwork. This study will provide a deeper understanding of the role of institutional context in providing adequate support to facilitate interdisciplinary team care delivery.

Confidentiality, Records, \& Distribution: All digital audio recordings and electronic documents reviewed will be stored securely on the Portland State server. Digital audio files will be created with a portable digital recorder, and then transferred to the H: drive. Paper documents and printed transcripts will be kept in a locked filing cabinet in the principal investigator's cubicle in the Urban Center, at the Mark O. Hatfield School of Government. These records will be kept for a minimum of 3 years following the conclusion of the dissertation, upon completion of degree the records will remain with me, in a locked cabinet.

All subjects who are recorded will be aware that the interviews are being recorded and special attention will be paid to let them know that the recordings are for analysis purposes only and the recordings will not be heard by anyone but the researcher. Individuals will have the opportunity to opt-out of a recording, and written notes will be taken instead. The principal investigator will be the only one with access to these recordings; however, these recordings may be made accessible to the dissertation committee if necessary (but ideally not).

Training and Experience: The researcher has been involved in qualitative research and evaluation for ten years. Most recently, I participated in the writing of an approved 
HSRRC application (HSRRC \#111904), approved October 2011. That particular application was for an evaluation that also involved interviews, as well as other qualitative collection methods. I have been through an IRB training at OHSU (2009), as well as successfully completed the OHSU review process five times from 2003-2009. Although this is my first application as the primary researcher, I have been through the review process and contributed to several applications in the past. As a doctoral candidate, my chair Sherril Gelmon, DrPH and other committee members will advise me: Paula Carder, PhD, Gregory Schrock, PhD, and Donald Truxillo, PhD. 


\section{Appendix B: Initial Email for Organizational Approval}

Dear [PACE leadership contact]:

I am a Doctoral Candidate in the Public Affairs and Policy $\mathrm{PhD}$ program at the Mark O. Hatfield School of Government at Portland State University, in Portland, OR. My dissertation chair is Dr. Sherril Gelmon, Chair of the Division of Public Administration. My dissertation is about the institutional context that supports interdisciplinary teamwork in the care of older adults. I am doing a multi-site case study with five PACE programs across the country.

I would like to study your PACE program as a part of this research. I used a selection hierarchy that helped me identify your program as a good candidate for this study. Your selection was based on demographic variation, organizational form, and state health policy environment. I am seeking to interview 5 individuals within each organization: the executive director (or equivalent), one PACE team member, one member of the governing body, and then 1-2 other interviews that will be identified by individuals who have already been interviewed. These may include the quality assurance and improvement representative, a member of the participant advisory committee, former program or medical directors, or team leaders.

If you agree, I would like to set an appointment with you at the time and date you are free. I can answer any further questions you may have and learn of any other processes or paperwork required for your participation. You can directly contact me by email (foucek@pdx.edu) or phone (xxx-xxx-xxxx).

I hope to hear from you soon. Thank you.

Regards,

Anna Foucek Tresidder, MPH 


\section{Appendix C: Initial Email for Key Informant Recruitment \\ Dear [PACE affiliated informant]:}

I am a Doctoral Candidate in the Public Affairs and Policy PhD program at the Mark O. Hatfield School of Government at Portland State University, in Portland, OR. My dissertation chair is Dr. Sherril Gelmon, Chair of the Division of Public Administration. My dissertation is about the institutional context that supports interdisciplinary teamwork in the care of older adults. I am doing a multi-site case study with five PACE programs across the country.

I would like to interview you as a part of my study. I have been in touch with the [CEO or Director] of your institution who has given approval for your participation in this research. Your name was given to me by [National PACE Association, other PACE employee, access through website]. The interview will take about 90 minutes and will be conducted [in-person, over the phone, or via Skype].

If you agree, I would like to set an appointment with you at the time and date you are free. If you have questions, you can directly contact me by email (foucek@pdx.edu) or phone $(\mathrm{xxx}-\mathrm{XxX}-\mathrm{Xxxx})$.

I hope to hear from you soon. Thank you.

Regards,

Anna Foucek Tresidder, MPH 


\section{Appendix D: Informed Consent Form}

On Portland State University letterhead

$\underline{\text { Interdisciplinary Teams in the Care of Older Adults }}$

You are invited to participate in a research study conducted by Anna Foucek Tresidder, who is a Doctoral Candidate in the Public Affairs and Policy PhD Program at the Mark O. Hatfield School of Government at Portland State University. I hope to learn how your organization supports interdisciplinary team care within your PACE program. This study is part of the requirements for completion of my doctoral degree. My doctoral committee chair is Sherril Gelmon, DrPH, who is the supervising faculty of this work.

If you decide to participate, you will be asked to participate in an interview which will take place either face-to-face, via Skype, or over the phone. The interview will take approximately 90 [60 for some respondents] minutes. This conversation will be digitally recorded unless you object to recording. While participating in this study there is minimal risk to you and you can decline to answer any questions that do you not wish to answer. You may not receive any direct benefit from taking part in this study, but the study may help to increase knowledge within your organization, which may also help other organizations in the future. All information that is obtained in connection with this study and that can be linked to you or identify you in any way will be kept confidential. All reporting will be with identities blinded to ensure anonymity. The recordings are for analysis purposes only and the recordings will not be shared with anyone but my 
dissertation committee. You will have the opportunity to opt-out of the recording, and written notes will be taken instead.

Your participation is voluntary. You do not have to take part in this study, and it will not affect your relationship with your employer, PACE program staff or Portland State University. If you have any questions about your participation in this study, please contact Anna Foucek Tresidder at (xxx-xxx-xxxx), or foucek@pdx.edu. You may also contact my dissertation chair, Dr. Sherril Gelmon, at (503) 725-3044, or gelmons@pdx.edu. If you have concerns about your rights as a research subject, please contact Human Subjects Research Review Committee, Research and Strategic Partnerships (RSP), PO Box 751, Portland, OR 97207, 503-725-4288 (1-877-480-4400), hsrrc@pdx.edu.

Your signature indicates that you have read and understand the above information and agree to take part in this study. The researcher will provide you a copy of this form for your records.

Signature

Date

Print Name 


\section{Appendix E: Interview Protocol for Executive/Medical/Program Director}

The following will be said at the beginning of the interview:

"Thank you for making the time to talk with me today for this interview about institutional context and PACE interdisciplinary teams (IDTs). As I explained in my invitation to this interview, I am conducting 25 interviews for this study; five will originate from this PACE program. These interviews are being conducted with key leadership, management, and staff to gain insights about the institutional context of the PACE program and how it supports its IDT.

This interview is part of my work for my doctoral dissertation at Portland State University, through which I hope to illuminate the institutional context that supports and legitimizes interdisciplinary team practice in the care of the frail elderly. This study will explore how federal, state, and local policies influence the development and support of the IDT model. Elements will be examined at the organization and program levels, as well as the team level.

You are invited to participate in this interview as a key informant who has insights that can inform this work. You have been identified as a key informant by [examination of organization documents, National PACE Association, or member of PACE staff] because of your experience as a [current job title, past job title, relationship to PACE organization].

I appreciate you making time to share your perspectives with me. The interview should take about 90 minutes. With your permission, I will be taking notes and recording 
our conversation. Your participation in this interview is voluntary and you may stop the interview at any time. You do not have to answer any questions that you do not want to answer. All individual responses will be kept anonymous in my reporting, and complete confidentiality of your responses is assured.

The intent of this conversation is to help identify key insights related to the institutional context within which the interdisciplinary team function from the perspective of multiple informants associated with a PACE program. The notes and recordings will assist me in accurately representing our discussion, but no one other than me will hear the recordings or read the transcript. If there are things that you do not want repeated, please indicate this so that I do not include the comment in the final write up. Comments and quotes will not be attributed to an individual. The detailed descriptions of this and other cases will be synthesized and analyzed in my dissertation.

At this time, do you have any questions or concerns? [Allow time to answer...]

Did you have time to review and sign the consent form? Do you have any questions about it? [Establish how the signed copy will be delivered to the researcher, if interview is done remotely]

All the questions I ask should be answered from your point of view only. You should feel no obligation to speak or represent anyone other than yourself and your position within the organization.

Let's begin..." 
First, I would like to ask you about your organization and your role in it.

1. Please describe the goals and mission of your organization. [5 minutes]

a. Probe: What are the organization's overall values?

b. Probe: How do you the organization's goals/mission/values factor into your job/role?

2. What is your role/relationship to the PACE interdisciplinary (IDT) team? [5 minutes]

For the next questions, let us focus on context elements that may support or create obstacles to IDT functioning.

3. From your point of view does the IDT culture within the PACE program closely resemble the organizational culture? Please explain. [7 minutes]

4. How is the approach to leadership decided within the team? [10 minutes]

a. Probe: Are there strategies to prevent defaulting to a hierarchical power structure within the team?

b. Probe: To what extent is the team involved in the process?

5. How has your organization been able to work within its policy and regulatory framework to affect IDT functioning? [11 minutes]

a. Probe: Does your state or organization utilize waivers to change the scope of PACE services?

b. Probe: How are responses developed to issues that arise in relation to care quality and team function?

6. How does your organization minimize the potential for conflict between professions within the IDT? [11 minutes]

a. Probe: Are there compulsory team-building activities?

b. Probe: How do you assure the inclusion of all team members in defining roles and processes for team functioning?

7. What organizational characteristics have influenced the time and space that IDTs have to meet and collaborate in decision-making around patient care? [11 minutes]

a. Probe: Are routines formalized? 
b. Probe: Is equivalent technology available to all IDT members? How are boundaries drawn around technology access?

c. Probe: Are there issues of urgency and complexity that compromise team functioning?

The next questions specifically address the quality improvement activities that focus on team function.

8. How do you measure successful team function within your organization? [7 minutes]

a. Probe: How do you address conflicts between the team and the organization?

b. Probe: How do you address conflicts within the team?

9. Is there ongoing team or organizational development activities designed to improve team functioning? [ 8 minutes]

a. Probe: Are there any specific activities to improve communication?

b. Probe: are there activities on how to address patient satisfaction or outcomes?

My final questions are about how the development of the PACE program has affected your organization.

10. What is the value added for your organization, in your opinion, for the increasing the acceptance of team-based patient care, since the opening of the PACE program? [7 minutes]

11. Does the PACE model influence any other areas of care delivery? [8 minutes]

a. Probe: Has there been consideration to providing PACE services to other populations? Has it been considered with chronic care or pediatrics?

b. Probe: Has the PACE program influence organization level social service coordination or provider collaboration?

Would it be all right if I contacted you later to ask for further clarifications?

Thank you very much for your time and insights! 


\section{Appendix F: Interview Protocol for Team Members}

The following will be said at the beginning of the interview:

"Thank you for making the time to talk with me today for this interview about institutional context and PACE interdisciplinary teams (IDTs). As I explained in my invitation to this interview, I am conducting 25 interviews for this study; five will originate from this PACE program. These interviews are being conducted with key leadership, management, and staff to gain insights about the institutional context of the PACE program and how it supports its IDT.

This interview is part of my work for my doctoral dissertation at Portland State University, through which I hope to illuminate the institutional context that supports and legitimizes interdisciplinary team practice in the care of the frail elderly. This study will explore how federal, state, and local policies influence the development and support of the IDT model. Elements will be examined at the organization and program levels, as well as the team level.

You are invited to participate in this interview as a key informant who has insights that can inform this work. You have been identified as a key informant by [examination of organization documents, National PACE Association, or member of PACE staff] because of your experience as a [current job title, past job title, relationship to PACE organization].

I appreciate you making time to share your perspectives with me. The interview should take about 90 minutes. With your permission, I will be taking notes and recording 
our conversation. Your participation in this interview is voluntary and you may stop the interview at any time. You do not have to answer any questions that you do not want to answer. All individual responses will be kept anonymous in my reporting, and complete confidentiality of your responses is assured.

The intent of this conversation is to help identify key insights related to the institutional context within which the interdisciplinary team function from the perspective of multiple informants associated with a PACE program. The notes and recordings will assist me in accurately representing our discussion, but no one other than me will hear the recordings or read the transcript. If there are things that you do not want repeated, please indicate this so that I do not include the comment in the final write up. Comments and quotes will not be attributed to an individual. The detailed descriptions of this and other cases will be synthesized and analyzed in my dissertation.

At this time, do you have any questions or concerns? [Allow time to answer...]

Did you have time to review and sign the consent form? Do you have any questions about it? [Establish how the signed copy will be delivered to the researcher, if interview is done remotely]

All the questions I ask should be answered from your point of view only. You should feel no obligation to speak or represent anyone other than yourself and your position within the organization.

Let's begin..." 
First, I would like to ask you about your organization and your role in it.

1. Please describe the goals and mission of your organization. [5 minutes]

a. Probe: What are the organization's overall values?

b. Probe: How do you the organization's goals/mission/values factor into your job/role?

2. What is your role/relationship to the PACE interdisciplinary (IDT) team? [2 minutes]

For the next questions, let us focus on context elements that may support or create obstacles to IDT functioning.

3. From your point of view does the IDT culture within the PACE program closely resemble the organizational culture? Please explain. [7 minutes]

4. What are the common beliefs [shared logics or working patterns] amongst the IDT? [7 minutes]

5. How does the IDT define its goals and objectives? How does it select the appropriate ways to pursue them? [7 minutes]

6. How is the approach to leadership decided within the team? [8 minutes]

a. Probe: Are there strategies to prevent defaulting to a hierarchical power structure within the team?

b. Probe: To what extent is the entire team involved in the process?

7. What are the other professional boundaries that influence goal accomplishment? [7 minutes]

8. Who is responsible for action when a patient or the family is dissatisfied with their care? [8 minutes]

9. How does your organization minimize the potential for conflict among the professions within the IDT? [8 minutes]

a. Probe: Are there compulsory team-building activities?

b. Probe: How do you assure the inclusion of all team members in defining roles and processes for team functioning?

10. What organizational characteristics have influenced the time and space that IDTs have to meet and collaborate in decision-making around patient care? [8 minutes] 
a. Probe: Are routines formalized?

b. Probe: Is equivalent technology available to all IDT members? How are boundaries drawn around technology access?

c. Probe: Are there issues of urgency and complexity that compromise team functioning?

The next questions specifically address the quality improvement activities that focus on team function.

11. How is successful team function measured within your organization? [5 minutes]

a. Probe: How do you address conflicts between the team and the organization?

b. Probe: How do you address conflicts within the team?

12. Probe: What are the continuing education opportunities for the organization about teamwork? Are there continuing education opportunities for the entire team? What CE opportunities are there for individuals? [5 minutes]

13. Is there ongoing team or organizational development activities designed to improve team functioning? [5 minutes]

a. Probe: Are there any specific activities to improve communication?

b. Probe: are there activities on how to address patient satisfaction or outcomes?

My final questions are about how the development of the PACE program has affected your organization.

14. Does the PACE model influence any other areas of care delivery? [8 minutes]

a. Probe: Has there been consideration to providing PACE services to other populations? Has it been considered with chronic care or pediatrics?

b. Probe: Has the PACE program influence organization level social service coordination or provider collaboration?

Would it be all right if I contacted you later to ask for further clarifications?

Thank you very much for your time and insights! 